Integrated Metabolomics, Transcriptomics, and Signal Protein Profile Define the Effector Function and Associated Metabotype of Five Polarized Macrophage Phenotypes

Catherine B. Anders Ph.D.*, Tyler M.W. Lawton*, Hannah L. Smith*†, Jamie Garret* ${ }^{*}$, Margaret M. Doucette DO*, and Mary Cloud B. Ammons Ph.D.*

* Idaho Veteran's Research and Education Foundation (IVREF); Boise VA Medical Center (BVAMC), Boise, ID 83702; USA

* Department of Physical Medicine \& Rehabilitation, Boise VA Medical Center (BVAMC), Boise, ID 83702; USA

$\dagger$ Department of Microbiology and Immunology; Montana State University, Bozeman, MT, ZIP 59717; USA † School of Medicine, University of Washington, Seattle, WA, ZIP 98195; USA

Corresponding Author: Mary Cloud B. Ammons Ph.D.

\title{
Summary Sentence
}

Macrophage functional plasticity of six macrophage phenotypes correlates with unique distinctions in cellsurface marker expression, signal protein secretion, transcriptomics profiles, and metabolic processes.

Key Words: Macrophage, Immunomodulation, Metabolism, Plasticity 
Abbreviations

$\alpha \mathrm{KG}$

AHS

ALA

AMP

ANOVA

APC

APCs

ARG

ASP

ATP

BCAA

bFGF

BSA

CCL

CD

CIT

CSV

CXCL

CYS

DAMPs

DGS

ECM

EGF

FAO

FAs

FASN

FDR

FGF

FITC

FMO

Fcy

GLU

GLY

GM-CSF

GO

GSEA

$\mathrm{Hb}$

$\mathrm{HC}$

HESI-II

HIF

HIS

HLA-DR alpha ketoglutarate

autologous human serum

alanine

adenosine monophosphate

analysis of variance

allophycocyanin

antigen-presenting cells

arginine

aspartate

adenosine triphosphate

branch chain amino acid

basic fibroblast growth factor

bovine serum albumin

chemokine (C-C motif) ligand

cluster of differentiation

citruline

comma-separated value

chemokine (C-X-C motif) ligand

cysteine

damage-associated molecular patterns

directed global significance

extracellular matrix

epidermal growth factor

fatty acid oxidation

fatty acids

fatty acid synthase

false discovery rate

fibroblast growth factor

fluorescein isothiocyanate

fluorescence minus one

fragment crystallizable gamma region

glutamate

glycine

granulocyte-macrophage colony-stimulating factor

gene ontology

gene set enrichment analysis

hemoglobin

hierarchical clustering

heated electrospray ionization

hypoxia-inducible factor

histidine

human leukocyte antigen - DR isotype 


\begin{tabular}{|c|c|}
\hline ICs & immune complexes \\
\hline IFN- $\gamma$ & interferon gamma \\
\hline IL & interleukin \\
\hline IL-1Ra & IL-1 receptor agonist \\
\hline IRB & institutional review board \\
\hline LIF & leukemia inhibitory factor \\
\hline LPS & lipopolysaccharides \\
\hline M-CSF & macrophage colony stimulating factor \\
\hline MEM & minimum essential media \\
\hline MerTK & Mer receptor tyrosine kinase \\
\hline MFI & median fluorescence intensity \\
\hline MHC-II & major histocompatibility complex class II \\
\hline MMP & matrix metalloproteases \\
\hline MRC1 & mannose receptor \\
\hline MФs & macrophages \\
\hline NADPH & nicotinamide adenine dinucleotide phosphate \\
\hline NO & nitric oxide \\
\hline OPLSDA & orthogonal PLSDA \\
\hline OXPHOS & oxidative phosphorylation \\
\hline PBMCs & primary blood-derived mononuclear cells \\
\hline PBS & phosphate buffered saline \\
\hline PCA & principal component analysis \\
\hline PDGF & platelet-derived growth factor \\
\hline $\mathrm{PE}$ & phycoerythrin \\
\hline PerCP-Cy & peridinin chlorophyll protein complex cyanine \\
\hline PFPA & perfluoropentanoic acid \\
\hline PGF & placental growth factor \\
\hline PLSDA & partial least-squares to latent structures discriminant analysis \\
\hline PPP & pentose phosphate pathway \\
\hline PRO & proline \\
\hline QC & quality control \\
\hline RNS & reactive nitrogen species \\
\hline ROS & reactive oxygen species \\
\hline RPMI & Roswell Park Memorial Institute \\
\hline RSD & relative standard deviation \\
\hline SDEGs & significantly differentially expressed genes \\
\hline $\mathrm{SDH}$ & succinate dehydrogenase \\
\hline SER & serine \\
\hline SLE & systemic lupus erythematosus \\
\hline SYK & spleen tyrosine kinase \\
\hline SPMs & specialized pro-resolving mediators \\
\hline SUS & Shared and Unique Structure \\
\hline $\mathrm{T}$ helper cells & Th cells \\
\hline
\end{tabular}


TAMs

TCA

TGF- $\beta$

THR

TLR

TNF

TXN

UDS

UHPLC/MS/MS

VEGF tumor associated MФs

tricarboxylic acid cycle

transforming growth factor- $\beta$

threonine

toll-like receptor

tumor necrosis factor

thioredoxin

undirected global significance

ultra-high-performance liquid chromatography/tandem accurate mass spectrometry

vascular endothelial growth factor

\section{Abstract}

Macrophages (MФs) display remarkable plasticity and the ability to activate diverse responses to a host of intracellular and external stimuli. Despite extensive characterization of M1 MФs and a broad set of M2 MФs, comprehensive characterization of metabolic shifts driving $М \Phi$ activation remains. Herein, we utilized an $e x$ vivo model to produce six $\mathrm{M} \Phi$ functional phenotypes. Isolated CD14+ PBMCs were differentiated into resting M0 MФs, and then polarized into M1 (IFN- $\gamma /$ LPS), M2a (IL-4/IL-13), M2b (IC/LPS), M2c (IL-10), and M2d (IL-6/LIF) МФs. The МФs were profiled using a bioanalyte matrix of four cell surface markers, $\sim 50$ secreted proteins, $\sim 800$ expressed myeloid genes, and $\sim 450$ identified metabolites relative to M0 MФs. Signal protein and expressed gene profiles grouped the MФs into inflammatory (M1 and M2b) and wound resolution (M2a, M2c, and M2d) phenotypes; however, each had a unique metabolic profile. While both M1 and M2b MФs shared metabotype profiles consistent with an inflammatory signature; key differences were observed in the TCA cycle, FAO and OXPHOS. Additionally, M2a, M2c and M2d MФs all profiled as tissue repair MФs; however, metabotype differences were observed in multiple pathways including hexosamine, polyamine, and fatty acid metabolism. These metabolic and other key functional distinctions suggest phagocytic and proliferative functions for M2a MФs, and angiogenesis and ECM assembly capabilities for M2b, M2c and M2d MФs. By integrating metabolomics into a systems analysis of $M \Phi$ phenotypes, we provide the most 
comprehensive map of $\mathrm{M} \Phi$ diversity to date, along with the global metabolic shifts driving $\mathrm{M} \Phi$ functional plasticity in these phenotypes.

\section{Introduction}

Macrophages (MФs) display remarkable functional plasticity and the ability to activate diverse responses to a host of intracellular triggers, external stimuli, and nutritional availability. Generally, MФs are divided into M1(classically activated, pro-inflammatory) MФs and a broad set of M2 (alternatively activated, antiinflammatory) MФs. M2 MФs have been further subdivided into M2a, M2b, M2c, and M2d subtypes [1]. These M2 subtypes have exhibited numerous, often overlapping, effector functions [2], and have emerged as keystone species in the functional ecology of such diverse environments as wounds [3], tumors [4-6], developing fetuses [7], and systemic autoimmune diseases [8, 9]. Comprehensive, functional characterization of such a diverse array of $\mathrm{M} \Phi$ populations presents an immense challenge and requires utilization of a systems-based approach that integrates transcriptional, proteomic, and metabolomic profiles to fully characterize these diverse MФ phenotypes. The emergent field of metabolomics has established a pivotal role for metabolism in immunomodulation of MФ polarization and functional phenotypes (reviewed in O-Neill, et al. [10]); yet the majority of this research is limited to comparing the more established M1 and M2a phenotypes, leaving much to be learned about the other known M2 MФ subtypes (i.e., M2b, M2c, and M2d).

Classically activated, pro-inflammatory M1 MФs are polarized by exposure to IFN- $\gamma$, TNF, LPS, and/or other antimicrobial signals that trigger TLR ligation [11]. M1 MФs exhibit increased and sustained proinflammatory responses including production of such anti-microbial molecules RNS and ROS, secretion of inflammatory mediators including IL-6, IL-1 $\beta$, IL-12, IL-18, and TNF $\alpha$ [12]. The upregulation of MHC-II and T-cell priming surface markers (e.g., CD40, CD80, and CD86), as well as chemokine ligand production (e.g., CXCL9, CXCL10, and CXCL11) in M1 MФs promote Type I immunity through Th1 and Th17 cytotoxic adaptive immune responses [12]. Although essential for defense against infection, continuous M1 polarization 
of MФs can lead to impaired wound healing and significant collateral tissue damage from sustained inflammation. Phagocytosis of apoptotic cells, detection of markers known as DAMPs or alarmins, and production of IL-5, IL-4, and IL-13 by nearby cells dampens M1-driven inflammation and facilitates the onset of an anti-inflammatory, tissue repair response dominated by M2 M $\Phi$ functional phenotypes [13].

M2 MФs (i.e., M2a, M2b, M2c, and M2d) known to represent the opposite end of the polarization spectrum from M1 MФs, exhibit tissue-reparative, pro-fibrotic, pro-angiogenic, and phagocytic functions [14-16]. Associated with Type 2 immunity and Th cell priming, M2 MФs are proposed to counteract the inflammatory microenvironment created by the M1 MФ phenotype [14]. M2a MФs, polarized by exposure to IL-4 and IL-13, have been described as expressing high levels of MHC-II, MRC1, CD36, and IL-1Ra to promote Th cell activation and suppress IL-1 mediated inflammation [14, 17]. Additionally, M2a MФs have been shown to secrete chemokines, fibronectins, and growth factors (e.g. FGF and TGF- $\beta$ ) to initiate cellular proliferation, recruit tissue-repair cellular populations, promote angiogenesis, and support development of myofibroblasts $[18]$.

M2b MФs, polarized by exposure to LPS and immune complexes (ICs), have been shown to crosslink Fc $\gamma$ receptors via ICs, inducing elevated CD80/CD86 expression for T-cell priming, promotion of Type 2 immunity, and high IL-10 and low IL-12 secretion [14, 19, 20]. Observed to be ineffective at bacterial clearance [21], M2b MФs display characteristics indicative of a tissue remodeling functional phenotype which include ECM synthesis and angiogenic potential [22].

Resting MФ exposure to IL-10, glucocorticoids, or TGF- $\beta$, drives polarization towards the M2c M $\Phi$, and has been characterized as having increased expression of the MerTK, surface display of CD163, secretion of PGF, and activation of MMPs. Based on this profile, M2c MФs are proposed to promote tissue repair through efferocytosis, ECM remodeling, and angiogenesis [15, 23]. While this phenotype appears to overlap with the M2a MФ functional phenotype, Gabriel, et al. (2014) demonstrated differences in the timing of gene expression during polarization and proposed that this temporal polarization may distinguish these $\mathrm{M} \Phi$ phenotypes in vivo 
[24]. Additionally, Jetten, et al. (2014) showed that while PGF signaling was necessary for M2c MФ-induced angiogenesis, PGF was not required for angiogenesis promoted by M2a MФs [18].

Tumor associated MФs (TAMs, referred to herein as M2d MФs), previously investigated within the tumor microenvironment, can be polarized through a variety of mechanisms including co-culture with cancer cells or ascites fluid, exposure to IL-6, LIF, and/or the purine nucleoside adenosine [16]. Associated with potent immunosuppressive functions and the promotion of angiogenesis [16, 25], M2d MФs have also been shown to secrete numerous proteolytic enzymes (e.g., MMP-2, MMP-9, and cathepsins), growth factors (e.g. EGF, VEGF. PDGF, and bFGF), and anti-inflammatory mediators (e.g., IL-10, TGF- $\beta$, B7-H1, B7-H3/H4) [4, 6, 16,

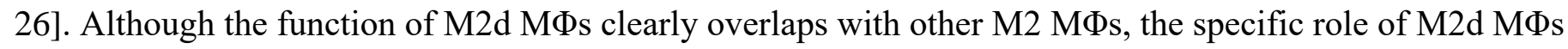
within the healing wound remains unknown.

While $M \Phi$ phenotypes have yet to be comprehensively described, the plasticity for M1/M2a M $\Phi$ has been demonstrated to depend on metabolic immunomodulation [10]. M1 MФ polarization favors aerobic glycolysis and glycolytic flux into the oxidative branch of the PPP, generating NADPH to feed the oxidative burst and serve as the building block for rapid biosynthesis [10]. Activation of PPP also supports the production of glutathione and other antioxidants to promote cellular longevity under the harsh conditions created by this proinflammatory МФ phenotype [27]. This anti-microbial function is further enhanced by a decoupled anaplerotic TCA with breaks at citrate and succinate. Accumulated citrate from the broken TCA cycle is diverted for the biosynthesis of fatty acids (FAs), prostaglandins, the anti-microbial metabolite itaconate, and the generation of NO [27-29]. Elevated itaconate inhibits SDH, resulting in succinate accumulation, $H I F$ stabilization, and $H I F$ mediated expression of pro-inflammatory cytokines $[27,30,31]$.

In contrast, M2a MФs utilize glycolysis to feed an intact TCA cycle coupled with FA oxidation (FAO), and active OXPHOS to generate ATP $[32,33]$. FAO also plays a role in reducing inflammation through reduced lipid accumulation and inhibition of pro-inflammatory mediators [34]. Additionally, M2a MФs rely heavily on glutamine to promote the hexosamine pathway needed for production of N-glycans and the upregulation of the 
mannose receptor [28]. Finally, arginine flux through ornithine contributes to collagen production and polyamine synthesis $[28,35]$.

Much less is known regarding metabolic immunomodulation of the remaining M2 M $\Phi$ phenotypes (i.e., M2b, M2c, and M2d). Rodriguez-Prados, et al. (2010) demonstrated that much like M1 and M2a MФs, M2c

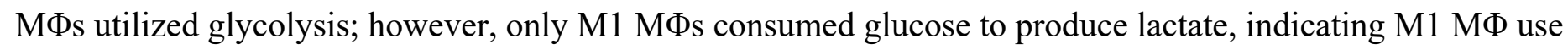
of aerobic glycolysis is unique and both M2a and M2c MФs utilize glycolysis to drive an intact TCA [30]. In contrast, M2a and M2c MФs could be distinguished based on glutamine consumption, suggesting a metabolic contribution to the unique mannose expression on M2a MФs [28]. Elevated glycolysis has also been

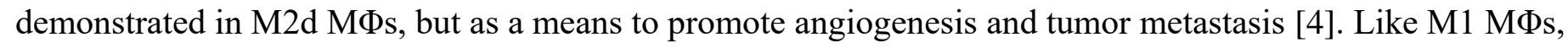
M2b cells have been shown to generate NO combined with decreased production of urea [14]. Alternately activated M2 MФs have an intact TCA, increased OXPHOS, low cellular energy (e.g. high AMP/ATP ratios), and elevated catabolism of FAs [36]; however the metabolic profiles of murine-derived MФs compared to human-derived MФs are inconsistent demonstrating how much is still unknown about how metabolism drives function in these diverse МФ phenotypes [37].

Multiple MФ functional phenotypes have been described in the literature (reviewed in Anders, et. al., 2019 [38]), yet the key components of metabolic immunomodulation that drive this functional diversity have not been well characterized. With a systems biology approach, we comprehensively examined an ex vivo MФ model that produced six functional phenotypes. Briefly, CD14+ PBMCs isolated from healthy human donors were differentiated into resting MФs (referred to as M0 MФs). MФ subsets were then polarized into M1 (IFN- $\gamma / \mathrm{LPS}$ ), M2a (IL-4/IL-13), M2b (IC/LPS), M2c (IL-10), and M2d (IL-6/LIF) MФ phenotypes. The MФs were then profiled using a bioanalyte matrix composed of four cell surface markers, 50 secreted cytokines/chemokines/growth-factors, $~ 800$ expressed myeloid genes and $\sim 450$ identified metabolites. By integrating metabolomics into a systems analysis of MФ phenotypes, we provide the most comprehensive map 
of $\mathrm{M} \Phi$ diversity to date, as well as the global metabolic shifts driving $M \Phi$ functional plasticity in these phenotypes.

\section{Materials and Methods}

\section{Human Subjects, Monocyte Isolation, and МФ Differentiation and Polarization}

Written informed consent was obtained from healthy human subjects (ages 18-60 years old) enrolled in the study approved by the VA Puget Sound institutional review board (IRB). Whole blood (100 mL total) was obtained through venipuncture; $70 \mathrm{~mL}$ of blood was drawn into $60-\mathrm{mL}$ syringe tubes pretreated with heparin (Fresenius Kabi USA, LLC; Lake Zurich, Illinois, USA) while $30 \mathrm{~mL}$ was collected into BD Vacutainer Serum tubes (Franklin Lakes, NJ, USA). The vacutainer serum tubes were incubated at room temperature for 30-60 minutes before centrifugation at $3500 \mathrm{rpm}$ for 20 minutes and separated serum was collected and stored at $-20^{\circ}$ C. PBMCs were obtained via Ficoll-paque density centrifugation (Ficoll-Paque Plus, GE Healthcare BioSciences; Uppsala, Sweden) followed by CD14+ monocyte isolation via negative immunomagnetic selection using the Pan Monocyte Isolation Kit (Miltenyi Biotech; Bergisch Gladbach, Germany). Monocyte culture media was made of Roswell Park Memorial Institute (RPMI) 1640 (Corning; Manassa, VA, USA) modified with $1 \%(\mathrm{v} / \mathrm{v})$ each of minimum essential media (MEM) non-essential amino acids (Gibco; Grand Island, NY, USA), sodium pyruvate (Gibco; Grand Island, NY, USA), L-glutamine (Lonza; Walkersville, MD, USA) and supplemented with $10 \%$ (v/v) autologous human serum (AHS; pooled from a minimum of four healthy human donors) and $50 \mathrm{ng} / \mathrm{mL}$ of recombinant human MФ colony-stimulating factor (M-CSF) (Biolegend; San Diego, CA, USA). Monocytes were cultured in monocyte culture media at a cellular density of $1.5 \times 10^{6}$ cells $/ \mathrm{mL}^{\mathrm{in}} 6-$ well cell culture plates. and incubated at $37^{\circ} \mathrm{C}$ with $5 \% \mathrm{CO}_{2}$. Monocytes received fresh modified culture media on day 4 and day 7 and were treated with predefined polarization stimuli to achieve the desired M $\Phi$ phenotype. Cells exposed to M-CSF alone produce the M0 phenotype. Classically activated M1 phenotype was achieved by exposure to 100 ng/mL LPS from Escherichia coli O111:B4 (Sigma Aldrich; St. Louis, MO, USA) and 20 
ng/mL recombinant human IFN- $\gamma$ (PeproTech; Rock Hill, NJ, USA). Alternately M2 MФs were activated as follows: M2a MФs from stimulation with 20 ng/mL recombinant human IL -4 (PeproTech; Rock Hill, NJ, USA) and 25 ng/mL recombinant human IL-13 (PeproTech; Rock Hill, NJ, USA); M2b cells with 100 ng/mL LPS and $16.7 \mu \mathrm{L} / \mathrm{mL}$ immune complexes (IC; see details below); M2c cells with $25 \mathrm{ng} / \mathrm{mL}$ recombinant human IL-10 (PeproTech; Rock Hill, NJ, USA); and M2d MФs from stimulation with and 50 ng/mL recombinant human IL-6 (R\&D Systems; Minneapolis, MN, USA) and $25 \mathrm{ng} / \mathrm{mL}$ recombinant human leukemia inhibitory factor (LIF; R\&D Systems; Minneapolis, MN, USA). ICs for M2b polarization were generated by mixing $1 \mu \mathrm{L}$ of a $1.32 \mathrm{mg} / \mathrm{mL}$ solution of bovine serum albumin (BSA; MP Biomedicals; Auckland, New Zealand) with 50 $\mu \mathrm{L}$ anti-albumin (bovine serum), rabbit IgG fraction polyclonal antibodies (Invitrogen; Eugene, OR, USA) and incubating at $37^{\circ} \mathrm{C}$ for one hour. This IC mixture, stable at $4^{\circ} \mathrm{C}$ for several days, was stored until needed. Polarized cells were incubated at $37^{\circ} \mathrm{C}$ with $5 \% \mathrm{CO}_{2}$ from between 24 and 72 hours in accordance with established MФ differentiation protocols.

\section{Characterization of MФ Phenotypes}

\section{Flow Cytometry Analysis}

Flow cytometry was conducted to access differences in cell surface marker expression between the six different phenotypes. Preliminary experiments were conducted using CD14+ monocytes on 2-, 5- and 7-days post-plating to differentiate between monocytes, immature MФs, and mature M0 MФs and to identify gating regions for future $\mathrm{M} \Phi$ phenotype experiments. For $\mathrm{M} \Phi$ phenotype experiments, a panel of four fluorescent markers was employed including fluorescein isothiocyanate (FITC) mouse anti-human CD40 (BD Biosciences; San Jose, CA, USA), phycoerythrin (PE) mouse anti-human CD86 (BD Biosciences; San Jose, CA, USA), peridinin chlorophyll protein complex cyanine (PerCP-Cy $\left.{ }^{\mathrm{TM}} 5.5\right)$ mouse anti-human CD163 (BD Biosciences; San Jose, CA, USA) and allophycocyanin (APC) mouse anti-human human leukocyte antigen - DR isotype (HLA-DR) (BD Biosciences; San Jose, CA, USA). Unstained cells, single stained cells, and fluorescent minus one (FMO) controls were included for each analyzed phenotype to identify unstained cellular populations. All 
samples were evaluated on an Accuri C6 flow cytometer (BD Biosciences; San Jose, CA, USA) and the data analyzed using Kaluza 2.1 Analysis software (Beckman Coulter; Indianapolis, Indiana, USA).

\section{Intracellular Metabolite Isolation and Analysis}

With the goal of creating a comprehensive snapshot of cellular function, multiple experimental sample types were collected from each $\mathrm{M} \Phi$ phenotype during cell harvest to obtain transcriptomic, proteomic, and metabolomic data from each experimental trial. At the desired time point, the cellular supernatant, composed of cell culture media and any non-adherent cells, was removed from the cell culture well and placed into falcon tubes on ice. One mL of ice-cold phosphate buffered saline (PBS) was added to the adherent cell layers and then removed to the tubes containing the cellular supernatant and the tubes centrifuged at $2000 \mathrm{rpm}$ for 8 minutes. The collected supernatant was stored at $-80^{\circ} \mathrm{C}$ until further analysis. Pelleted cells were flash-frozen in liquid nitrogen and stored on ice, while the remaining adherent cells were quenched with $350 \mu \mathrm{L}$ of ice-cold $100 \%$ methanol (Honeywell; Muskegon, MI, USA) and $350 \mu \mathrm{L}$ of ice-cold ultrapure distilled water (Invitrogen; Grand Island NY, USA) and removed through gentle cell scraping and added to the cell pellet fraction. Following the addition of $700 \mu \mathrm{L}$ of ice-cold chloroform (Acros Organics; Thermo Fisher Scientific; Waltham, MA, USA), the collected cells were vortexed for 30 seconds-and were transferred to FastPrep® lysing matrix D tubes (MP Biomedicals; Auckland, New Zealand). To achieve cell lysis, the tubes were homogenized during two cycles of $40 \mathrm{~s}$ each at $4.0 \mathrm{~m} / \mathrm{s}$ with a $90 \mathrm{~s}$ delay between cycles utilizing the FastPrep-24TM $5 \mathrm{G}$ Homogenizer (MP Biomedicals; Auckland, New Zealand). The homogenized samples were centrifuged at $16,000 \mathrm{x}$ g for 5 minutes at $4^{\circ} \mathrm{C}$, and then placed immediately on ice. The polar (methanol/water) layer and non-polar (chloroform) layers were subsequently transferred to $1.5 \mathrm{~mL}$ protein low binding microcentrifuge tubes. These metabolite suspensions were lyophilized overnight without heat on a Thermo Scientific ${ }^{\mathrm{TM}}$ Savant ${ }^{\mathrm{TM}}$ ISS110 SpeedVac ${ }^{\mathrm{TM}}$ (Waltham, MA, USA) and stored at $-80^{\circ} \mathrm{C}$ until the samples were shipped to Metabolon for further analysis. The remaining interphase layer was flash-frozen in liquid nitrogen and stored at $-80^{\circ} \mathrm{C}$ until RNA extraction. 


\section{Cytokines, Chemokines and Growth Factors}

All cytokines, chemokines and growth factors were measured using the supernatant collected during the intracellular metabolite extraction at 24- and 72-hr post polarization. The analytes were evaluated utilizing multiplex magnetic bead-based immunoassays (ProcartaPlex ${ }^{\mathrm{TM}}$, Thermo Fisher Scientific; Waltham, MA, USA) per the manufacturer's instructions, and were run on a MAGPIX ${ }^{\circledR}$ instrument (Luminex Corporation; Austin, TX, USA).

\section{RNA Extraction}

RNA was purified utilizing the Qiagen RNAeasy® plus mini kit (Germantown, MD, USA). The lysing tubes containing the interphase layer from the metabolite were first thawed, if needed, on ice, resuspended in the RLT lysis buffer supplemented with $1 \%(\mathrm{v} / \mathrm{v})$ of $\beta$-mercaptoethanol and homogenized, as described above. Following lysis, the RNA was extracted and purified according to the manufacturer's instructions. The integrity of the extracted RNA was evaluated on an Agilent 2200 Tape Station (Santa Clara, CA, USA) and diluted to a final volume of $5 \mu \mathrm{l}$, at a concentration of $20 \mathrm{ng} / \mu \mathrm{L}$.

\section{Nanostring analysis}

The nCounter Gene Expression - Hs Myeloid v2 Panel CodeSet (NanoString Technologies; Seattle, WA, USA) was used to evaluate the expression of genes associated with myeloid innate immune system response. In brief, and according to NanoString's procedure, 100ng of each RNA sample was added to the CodeSet in hybridization buffer and incubated at $65^{\circ} \mathrm{C}$ for 16 hours. Purification and binding of the hybridized complexes were then carried out automatically on the nCounter Prep Station using magnetic beads derivatized with short nucleic acid sequences that are complementary to the capture and reporter probes. The hybridization mixture was first allowed to bind to the magnetic beads via the capture probe then washed to remove excess reporter probes and unhybridized DNA fragments. Probe complexes were then eluted off the beads and hybridized to magnetic beads complementary to the reporter probe followed by wash steps to remove excess capture probes. 
In the last step, the purified target-probe complexes were eluted off and immobilized in the cartridge for data collection which was carried out in the MAX System Digital Analyzer (NanoString Technologies; Seattle, WA, USA). Digital images were processed, and barcode counts tabulated in a comma-separated value (CSV) format. The raw count data was then normalized to the mean of positive control probes followed by RNA content normalization to the geometric mean of housekeeping genes in the CodeSet. Data normalization was performed using nCounter Analysis Software version 4.0 (NanoString Technologies; Seattle, WA, USA). Gene set enrichment analysis (GSEA) was utilized to determine which Nanostring-defined functional pathways were regulated within the individually polarized phenotypes. Two representations of these pathways are presented in the data. The first, undirected global significance (UGS) score, was utilized to identify which pathways were differentially regulated in each phenotype regardless of whether the genes were up- or down-regulated when compared to the M0 MФs. An extension of this score, the directed global significance statistics (DGS), measured the extent to which the genes were up- or down-regulated within the given pathway. In general, a positive pathway score for a specific phenotype indicates a predominance of genes that are up-regulated within the pathway as compared to those that are down-regulated; a negative pathway score indicates the reverse [39]. Additional analyses were conducted using bioDBnet Biological Database [40] and DAVID bioinformatics resources [41]. The top up-regulated genes within each activated phenotype were selected for gene ontology (GO) enrichment analysis. The GO processes significantly enriched $(p \leq 0.05)$ were then chosen for comparison. With further evaluation, processes common to all phenotypes and related to either specific diseases or ubiquitous to the immune systems were eliminated from the GO process list (e.g. GO:0045824: negative regulation of innate immune response).

\section{Metabolite Analysis}

All samples were analyzed by Metabolon (Morrisville, NC, USA) using four ultra-high-performance liquid chromatography/tandem accurate mass spectrometry (UHPLC/MS/MS) methods. The following is a summary of Metabolon's procedure. All methods utilized a Waters ACQUITY ultra-performance liquid chromatography 
(UPLC) and a Thermo Scientific Q-Exactive high resolution/accurate mass spectrometer interfaced with a heated electrospray ionization (HESI-II) source and Orbitrap mass analyzer operated at 35,000 mass resolution. Lyophilized samples were reconstituted in solvents compatible to each of the four methods and contained a series of standards at fixed concentrations to ensure injection and chromatographic consistency. One aliquot was analyzed using acidic positive ion conditions, chromatographically optimized for more hydrophilic compounds. In this method, the extract was gradient eluted from a C18 column (Waters UPLC BEH C182.1x100 mm, $1.7 \mu \mathrm{m}$ ) using water and methanol, containing 0.05\% perfluoropentanoic acid (PFPA) and 0.1\% formic acid. A second aliquot was also analyzed using acidic positive ion conditions that was chromatographically optimized for more hydrophobic compounds. In this method, the extract was gradient eluted from the same aforementioned C18 column using methanol, acetonitrile, water, $0.05 \%$ PFPA and $0.01 \%$ formic acid and was operated at an overall higher organic content. A third aliquot was analyzed using basic negative ion optimized conditions using a separate dedicated C18 column. The basic extracts were gradient eluted from the column using methanol and water, however with $6.5 \mathrm{mM}$ ammonium bicarbonate at $\mathrm{pH}$. The fourth and final aliquot was analyzed via negative ionization following elution from a HILIC column (Waters UPLC BEH Amide 2.1×150 mm, $1.7 \mu \mathrm{m}$ ) using a gradient consisting of water and acetonitrile with 10mM ammonium formate, $\mathrm{pH}$ 10.8. The MS analysis alternated between MS and data-dependent MS scans using dynamic exclusion. The scan range varied slighted between methods but covered $70-1000 \mathrm{~m} / \mathrm{z}$. Several types of controls were analyzed in concert with the experimental samples: a pooled matrix sample generated by taking a small volume of each experimental sample served as a technical replicate throughout the data set; extracted water samples served as process blanks; and a cocktail of quality control (QC) standards that were carefully chosen not to interfere with the measurement of endogenous compounds were spiked into every analyzed sample, allowed instrument performance monitoring and aided chromatographic alignment. Instrument variability was determined by calculating the median relative standard deviation (RSD) for the standards that were added to each sample prior to injection into the mass spectrometers. Overall process variability was determined by calculating the median RSD for all endogenous metabolites (i.e., non-instrument standards) 
present in $100 \%$ of the pooled matrix samples. Experimental samples were randomized across the platform run with QC samples spaced evenly among the injections.

Raw data was extracted, peak-identified and QC processed using Metabolon's hardware and software. Compounds were identified by comparison to library entries of purified standards or recurrent unknown entities. Biochemical identifications are based on three criteria: retention index within a narrow RI window of the proposed identification, accurate mass match to the library $+/-10 \mathrm{ppm}$, and the MS/MS forward and reverse scores between the experimental data and authentic standards. The MS/MS scores are based on a comparison of the ions present in the experimental spectrum to the ions present in the library spectrum. Peaks were quantified using area-under-the-curve. The resulting data was then normalized to cell count to account for differences in metabolite levels due to differences in the amount of material present in each sample.

\section{Metabolomics Data Analysis}

After normalization, peak intensity values were uploaded into MetaboAnalyst 4.0 (Ste. Anne de Bellevue, Quebec) [42], normalized using pareto scaling, and then statistically analyzed. Using MetaboAnalyst 4.0, partial least-squares to latent structures discriminant analysis (PLSDA) and orthogonal PLSDA (OPLSDA) were employed to characterize and visual metabolomic differences between the resting MO MФs and the individually polarized phenotypes. For detailed descriptions of OPLSDA utilization with metabolomics data, readers are referred to Wiklund, et al., (2008) [43]. PLSDA, a regression method, was employed to identify relationships between the UHPLC/MS/MS data (X) and binary vectors (Y) with a value of 0 for M0 resting MФs and 1 representing the polarized phenotype as they are compared individually to the M0 cells. Orthogonal PLS (OPLS) was then used to separate the systematic variation in $\mathrm{X}$ into two components: the first component, a predictive component (covariance), linearly associated with $\mathrm{Y}$, describes between phenotypic variance; and the second component (correlation), orthogonal to Y describes between sample variation [43].

To adequately demonstrate the OPLSDA results, three data visualization methods were utilized. The first, cross-validated score plots, display the cross-validated score values (Orthogonal T-score) on the y-axis versus 
the predictive or model T-score (T-score) on the $\mathrm{x}$-axis for each sample point within the analysis. S-Plots were used to visualize the predictive component of the model. The $\mathrm{x}$-axis represents the magnitude or covariance of each metabolite to the model while the reliability or correlation of those metabolites is plotted on the y-axis. Generally, metabolites that have covariance scores greater than 0.2 or less than -0.2 . and correlation values greater than 0.6 or less than -0.6 are considered to contribute significantly to the predictive value of the model. Shared and Unique Structure (SUS) plots were employed to compare the results from the M1 OPLS model to each M2 (M2a, M2b, M2c and, M2d) phenotype model. For example, by plotting the correlation from the predictive component from the M0 versus M1 model and the M0 versus M2a model, metabolic biomarkers can be identified that are not only uniquely correlated to the M1 and M2a MФs, but also have shared differentiation from the parent M0 phenotype. The correlation scores for each M2 subtype were plotted on the y-axis and the M1 scores on the x-axis. In this representation, metabolite features that are shared between phenotypes will cluster close to the diagonals while those features unique to each phenotype will fall outside of the diagonals.

\section{Online Supplemental Material}

Additional data provided in the Online Supplemental Material includes gene and protein expression of immunomodulatory factors (Supplemental Figure 1). Supplemental Figure 2 details global transcriptomics differences between each MФ functional phenotype and the parent M0 phenotype. Supplemental Figure 3 includes representations of PCA and OSPLDA multivariate analysis of the global transcriptomic data and a heatmap demonstrating metabolic differences between each $M \Phi$ functional phenotypes relative to the parent, resting macrophage phenotype. Additional phenotypic data for the for $\gamma$-glutamyl amino acids and various lipid subgroups are depicted in Supplemental Figure S4. Supplemental Tables S1 and S2 outline the relative genetic expression of select transcripts and the relative concentrations of select pathway metabolites for each polarized phenotype compared to M0 parent cells, respectively. 


\section{Results}

\section{Classification of MФ Phenotypes by Cell Surface Markers Provides Poor Resolution of Subtypes}

Six MФ phenotypic subpopulations were produced from CD14+ monocytes derived from human PBMCs by differentiated into resting MФs (referred to herein as M0 MФs) prior to polarization into M1 (IFN- $\gamma / \mathrm{LPS}$ ), M2a (IL-4/IL-13), M2b (IC/LPS), M2c (IL-10), and M2d (IL-6/LIF) MФ phenotypes. For simplicity of notation, these phenotypes will be referenced herein as M1, M2a, M2b, M2c, and M2d MФs. Following polarization, these MФ phenotypes were comprehensively profiled by cell-surface marker display, immunity-targeted proteomics, myeloid gene transcriptomics, and global metabolomics to obtain a systems-level functional profile for each phenotype.

Four MФ cell surface markers commonly associated with antigen-presentation or phagocytotic capacity of MФ phenotypes [44] were used as a first-pass characterization of phenotype function, namely CD40, CD86, CD163, and HLA-DR (Fig. 1). As shown in the histogram overlay of phenotype (Fig. 1 A-D) and associated MFI bar chart (Fig. 1E-H), pro-inflammatory M1 MФs (red bars) demonstrated elevated cell-surface display for CD40 (Fig 1A, E) and CD86 (Fig. 1B, F) compared to resting MФs (M0, blue bars). Within the alternatively activated MФ subgroups, only the M2a MФs (yellow bars) indicated APC functionality with higher display levels of both CD86 (Fig. 1B, F) and HLA-DR (Fig. 1D, H). Cell-surface display of CD163 (Fig. 1C, G) was elevated in both M2c (gray bars) and M2d MФs (purple bars), while all other phenotypes had decreased cellsurface display when compared to M0 MФs. Interestingly, M2b MФs (green bars) alone had decreased cellsurface display of HLA-DR when compared to the M0 parent phenotype (Fig. 1D, H). Taken together, M1 MФs profiled as CD40 ${ }^{\text {high }} \mathrm{CD} 866^{\text {high }} \mathrm{CD} 163^{\text {low }}$, M2a MФs as CD86 ${ }^{\text {high }} \mathrm{CD} 163^{\text {low }} \mathrm{HLA}-\mathrm{DR}{ }^{\text {high }}$, M2b MФs as CD163 ${ }^{\text {low }}$ HLA-DR ${ }^{\text {low }}$, while both M2c and M2d MФs appeared as CD86 ${ }^{\text {low }}$ CD163 ${ }^{\text {high }}$. Based on cell-surface display of these markers, APC functionality is primarily exhibited by M1 and M2a MФs, T-cell stimulation is dominated by M1 MФs, and phagocytic capacity was predominant in M2c and M2d MФs. Though common 
indicators of the broad functionality of MФs, these cell-surface display profiles clearly lack the resolution to distinguish between these six phenotypes, nor provide much insight into phenotypic functionality.

\section{Targeted Profiling of Immune-Mediator Profile by both Transcript and Protein Expression}

\section{Demonstrates MФ Functional Phenotype Along Pro-/Anti-Inflammatory Axis}

Cytokines, chemokines, and growth factors induced during $\mathrm{M} \Phi$ activation are involved in multiple effector functions, including the promotion or inhibition of inflammation. Post-polarization, M $\Phi$ culture supernatant was multiplex profiled for 22 immunomodulating proteins as depicted (Fig. 2A-C, bar charts, left y-axis and Supplemental Fig. 1A-G in the Online Supplementary Material). In parallel, total RNA was extracted from each MФ culture and profiled for mRNA expression (Fig. 2A-C, diamond-whisker plots, right y-axis and

Supplemental Fig. 1A-G). As IL-10, IL-4, IL-13, IFN $\gamma$, and IL-6 were used as polarizing factors for M2c, M2a, M1, and M2d MФs, respectively, an N/A was used for these specific phenotypes and respective protein concentration profiles.

As expected, M1 MФs secrete high levels of pro-inflammatory cytokines and chemokines including TNF $\alpha$, IL-12p70, CCL3, CXCL10, CXCL8, and IFNa (Fig, 2A); however, the M2b MФs, which are often classified under the alternately-activated M2 MФ phenotypes, also produced these pro-inflammatory factors, though to a lesser extent (Fig. 2A). Additionally, both M1 and M2b MФs phenotypes also secreted anti-inflammatory cytokines IL-4 (Supplemental Fig. 1D) and IL-13 (Supplemental Fig. 1E), demonstrated to mediate inflammatory responses and drive M2a MФ activation [45]. Other inflammation-mediating cytokines secreted

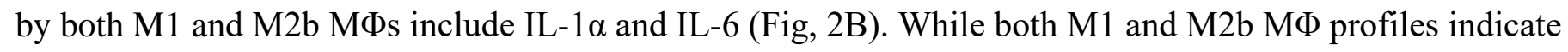
functionality on the pro-inflammatory side of the immune-modulation axis, M2b MФs are moderately different from M1 MФs in secretion of IL-10, TGF $\beta$, CCL2, and GM-CSF (Fig. 2B, C).

Of the other alternately activated M2 MФ phenotypes, M2a MФs are distinguished by secretion of CCL2 (Fig. 2B) and GM-CSF (Fig. 2C), while both M2a and M2b MФs produced IFN $\gamma$ (Supplemental Fig. 1F). While demonstrating similar patterns for most pro-inflammatory mediators, M2c MФs can be distinguished from M2d 
MФs by secreting a surprising level of IFN $\alpha$ while M2d MФs are exceptional in their high levels of TGF $\beta$

secretion (Fig. 2 A, B). Taken together, these protein profiles indicate that M1 and M2b MФs are significantly more active along the inflammatory axis than any of the other $M \Phi$ phenotypes and that phenotypic functionality for M2a, M2c, and M2d is much more restricted with regard to inflammation.

Finally, the mean mRNA expression (represented by the dark circles in the diamond-whisker plots) generally reflect trends observed in secreted protein levels with a few notable exceptions. Most dramatically, IL-1 $\beta$ and CXCL8 transcripts in M2b MФs are significantly upregulated when compared to the other phenotypes, but secreted protein levels are far less remarkable and significantly lower than the M1 MФ profile (Fig. 2 A, B). These striking differences indicate an important contribution of post-transcriptional regulation to phenotypic functionality, specifically in the M2b MФ phenotype.

\section{Global patterns of myeloid gene expression demonstrate impact of polarization and further define}

\section{functional phenotype as distinct from the parent, resting МФ phenotype}

Univariant, hierarchical clustering (HC) of gene expression levels revealed the modulatory effect polarization has on these MФ phenotypes. The most impactful dynamics in gene expression were observed in M1, M2a, and M2b MФs (Supplemental Fig. 2A). Interestingly, M1 and M2b clustered together, while all other M2 subtypes clustered more closely with their non-polarized parent phenotype M0. Of these M2 subtypes, M2a and M2c MФs formed unique clusters, while M2d MФs clustered together with M0 MФs. To further visualize polarization impacts on gene expression dynamics, the fold change for each gene was calculated relative to the parent M0 MФ phenotype and relative expression was displayed as the distribution across a logarithmic scale ($1 \leq \log _{2} \mathrm{FC} \geq 1$; Supplemental Fig. 2B). In general, the relative number of differentially expressed genes observed for each phenotype mirrors the patterns identified through $\mathrm{HC}$, with M1 and M2b MФs most transcriptionally active and M2a, M2c, and M2d decreasingly active in that order. Interestingly, the relatively increased transcriptional activity observed in both M1 and M2b MФs is primarily accounted for in upregulated gene expression when compared to the other M2 MФ subtypes, for which increased transcriptional activity is 
fairly evenly distributed between up and down regulated genes, further emphasizing the correlation between polarization and activation specific to $\mathrm{M} 1$ and $\mathrm{M} 2 \mathrm{~b} \mathrm{M} \Phi$ phenotypes.

ANOVA analysis using an adjusted p-value (false discovery rate [FDR]) of 0.05 revealed 189 SDEGs across all phenotypes and reanalysis by $\mathrm{HC}$ demonstrated that, when constrained to SDEG, the M2d phenotype more closely clusters with M2c MФs than their parent M0 MФs; however, M2c, M2d, and M0 MФs cluster separately from M2a MФs (Supplemental Fig. 2C). Supporting the HC pattern with SDEG constraints, multivariate clustering by PCA project M0, M2a, M2c, and M2d MФ phenotypes occupying overlapping 3D space, while M1 and M2b MФ phenotypes share a single dimension and are distinctly different from the other MФ phenotypes (Supplemental Fig. 3A). Accounting for $>90 \%$ of the variance in the data within the three components, only $7.4 \%$ of the variance is accounted for in the dimension separating the M1 and M2b MФ phenotypes, further emphasizing the similar impact of polarization on gene expression patterns in these two phenotypes.

Finally, while PCA provided cluster separation and variance across all six MФ phenotypes, use of OPLSDA provided clearer class separation of the polarized MФ phenotypes from their non-polarized, parent phenotype M0 in the cross-validated score plots (Supplemental Fig. 3B-F). Contribution of variance to phenotype cluster separation is plotted along the $\mathrm{x}$-axis and variance within phenotype is plotted along the $\mathrm{y}$-axis. Although variance within phenotype is notable for all phenotypes except M1 MФs, variance between the parent, nonpolarized M0 MФs and the polarized phenotypes is even more evident. In agreement with the SDEG constrained HC and PCA analyses, between phenotype variance is greatest for M1 MФs and M2b MФs, relative to M0 MФs, accounting for $440-45 \%$ of the total variance (Supplemental Fig. 3B, D), and nearly twice the

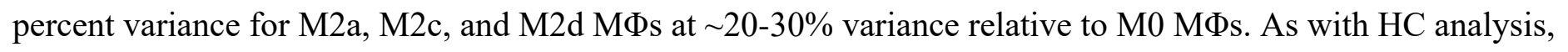
M2d MФ profiling is most akin to its parent M0 MФ phenotype, accounting for only $18.8 \%$ of the total variance (Supplemental Fig. 3F). Taken together, these data further demonstrate that when looking beyond the classic 
pro-/anti-inflammatory axis for defining $М \Phi$ phenotype, clear distinctions can be made that suggest phenotype plasticity includes a wide range of functionality in these cell subtypes.

\section{Pathway Analysis and GO Process Characterization Indicates Biological Functionality Within Each}

\section{Distinct МФ Phenotype}

Having demonstrated that polarization drove each $М \Phi$ phenotype towards distinct programs of myeloid gene expression, gene set enrichment analysis (GSEA) was utilized to determine how transcriptional activation translated into putative myeloid functionality as each phenotype progressed away from the resting, parent M0 MФ phenotype. Analysis by undirected global significance scores (UGS) displayed all detected pathways differentially impacted relative to the resting M0 parent MФs (displayed as solid colored plots in the radar graphs in Fig. 3). Consistent with the HC results, M1 and M2b MФ polarization most dramatically impacted pathways associated with myeloid cell functionality (Fig. 3A, C), while a less significant impact was observed for the M2a, M2c, and M2d MФs (Fig. 3B, D, E).

To better translate the impact of polarization into proposed biological functionality, the directed global significance statistic (DGS) measures the extent to which gene expression contributes to up- or down-regulation of the relevant biological pathway (displayed as white hashed plots in the radar graphs in Fig. 3). Positive DGS values that extend beyond the boundary of impact (thick maroon circle) denote biological pathways upregulated for that $\mathrm{M} \Phi$ phenotype, whereas negative DGS values retracted within this boundary denote biological pathways down-regulated for that $М \Phi$ phenotype. Highlighted SDEGs of relevance to these biological pathways are depicted in Supplemental Table 1. Of the 19 functionally defined, biological pathways, overall polarization is an "activation" process for M1, M2b, and M2d M $\Phi$ s with gene expression driving fifteen biological pathways into activation for M1 MФs and M2b MФs (Fig. 3 A, C) and eleven biological pathways

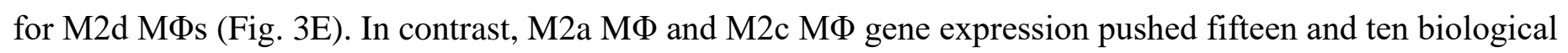
pathways into “inhibition”, respectively (Fig. B, D). 
M1 MФ polarization resulted in gene expression patterns that drove functionality in anticipated directions with up-regulation of antigen presentation, cell migration and adhesion, chemokine signaling, cytokine signaling, interferon signaling, pathogen response, and Th1-specific T-cell activation pathways, while angiogenesis, complement activation, and Th2-specific T-cell activation pathways are downregulated (Fig. 3A). GSEA identified 97 SDEGs $\left(\left(-1 \leq \log _{2} \mathrm{FC} \geq 1\right.\right.$ and $\left.\mathrm{p} \leq 0.05\right)$ contributing to $41 \%$ of IFN- $\gamma$ responsive and $12 \%$ LPS-responsive gene expression. Within the IFN- $\gamma$ responsive genes, interferon pathway genes, $I R F 1, I R F 7$, IRF8 and ISG15, are known transcriptional enhancers of genes associated with antigen presentation and Th1 responses (e.g. CD40 and CD86), cytokine and chemokine signaling (e.g. TNF, CXCL9, CXCL11, CCL4, CCL8, and STAT1), and pathogen response (e.g. IL18 and IRG1) [46]. These SDEGs and related upregulated pathways are consistent with defined pro-inflammatory and antimicrobial effector functions known to be associated with M1 MФ polarization.

While IFN- $\gamma$ and LPS are considered potent MФ activators, the IL-4 and IL-13 stimuli utilized for M2a polarization are considered mild polarization activators [47]. Consequently, when compared to the parent M0 MФ phenotype, M2a MФs exhibited a remarkably different pathway profile than the M1 MФs. While all 19 pathways were differentially regulated, only the $\mathrm{T}$-cell activation, $\mathrm{T}_{\mathrm{h}} 2$-specific $\mathrm{T}$-cell activation, metabolism, and ECM remodeling pathways were upregulated (Fig. 3B). Specifically, GSEA results showed that of the 83 identified SDEGs only $36 \%$ of these transcripts were upregulated with $84 \%$ being downregulated. Despite being upregulated when compared to M0 MФs, the T-cell and $\mathrm{T}_{\mathrm{h}} 2$ activation pathways did not exhibit any SDEGs using our inclusion criteria. For the ECM remodeling pathway, known metalloproteinase genes, ADAM19 and MMP12, were upregulated and the common M2a MФ-associated transcripts of TGM2, FABP4, and PTGS1 were also upregulated for the metabolism pathway, indicating changes in metabolic processes associated with M2a MФ polarization. The most upregulated genes, CCL17, CCl22 and CCL18, support the generally accepted role that $\mathrm{M} 2 \mathrm{a} \mathrm{M} \Phi$ s play in cellular proliferation, ECM remodeling, and tissue repair. 


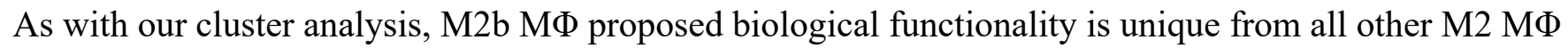
subtypes and most like the M1 MФ phenotype when compared to the resting M0 MФs. M2b MФ gene expression during polarization drives downregulation of antigen presentation, cell migration and adhesion, complement activation, and $\mathrm{T}_{\mathrm{h}} 2$-specific $\mathrm{T}$-cell activation response pathways (Fig. 3C). Chemokine signaling, the most upregulated pathway, is associated with prominent SDEGs and is intertwined with many other functional pathways observed for this phenotype. For example, chemokines CXCL2, CXCL3, and CXCL5 promote angiogenesis through the recruitment of angiogenic neutrophils and CCL5, CCL8, and CXCL9 support the recruitment and differentiation of myeloid cells necessary for tissue remodeling functions. While M2b M $\Phi$ polarization indicates biological functionality comparable to M1 MФs, distinct differences in such pathways as angiogenesis and cell migration and adhesion indicate that polarization of this phenotype may have a more localized biological relevance.

In a similar trend to M2a MФs, the pathway scores observed for M2c MФs imply that IL-10 is also a mild polarizing stimulus; however, patterns of pathway up- and down-regulation are distinctly different. Of the 19 biological pathways detected, nine had DGS scores indicating gene expression driving pathway upregulation compared to the M0 parent MФs (Fig. 3D). Key pathways included upregulation of angiogenesis, chemokine signaling, cytokine signaling, differentiation of myeloid cells, Fc receptor signaling, growth factor signaling, interferon signaling, lymphocyte activation, and T-cell activation (Fig. 3D). Interestingly, many of the identified SDEGs have broad effects that regulate inflammation (DUSP1, SOCS3, S100A8, and S100A9), vascular insult and neutrophil chemotaxis (S100A8 and S100A9), as well as protection against apoptosis and oxidative stress (IER3) [15]. Based on these pathway impact profiles, the biological functionality of polarization into this $M \Phi$ phenotype may serve to modulate immune response in the transition to resolution.

At first glance, M2c and M2d MФs appeared to polarize into phenotypes of similar biological functionality; however, polarization of MФs to the M2c phenotype resulted in a majority of down-regulated pathways and polarization of MФs to the M2d phenotype resulted in a majority of up-regulated pathways (Fig. 3D, E). Their 
transcriptional profile suggested overlapping effector functions such as resolution of inflammation (DUSP1, SOCS3, THBD, and CD163) and immune suppression (CCL8); however, upregulation of TLR signaling in M2d MФs during polarization is notably distinct from polarization in M2c MФs and M2d MФs differentially regulated many known pro-angiogenic markers such as ENPP2, SPHK1, CXCL2, CXCL3, and ID1, findings in line with a proposed biological role beneficial to survival of associated tumors.

While UGS and DGS scores provided both magnitude and directionality of polarization of each MФ phenotype overlaid on proposed biological functionality, GO annotation of global myeloid gene expression was utilized to provide a direct comparison between each polarized phenotype (Fig. 4). For each GO annotation, all five MФ phenotypes are displayed; however, the immediate similarity between M1 and M2b MФs can be observed when the GO annotation is selected for the top $15 \mathrm{GO}$ processes for M1 MФs and M2b MФs. GO annotation generally supported DGS analysis of polarization; however, interesting differences were noted. Specifically, secretion of IL-12, regulation of PDGF production, and response to NO were pathways identified as unique to M1 MФs (Fig. 4A), while regulation of leukocyte migration, neutrophil extravasation, and connective tissue replacement in inflammatory wound healing were pathways identified as unique to M2b M $\Phi$ (Fig. 4C).

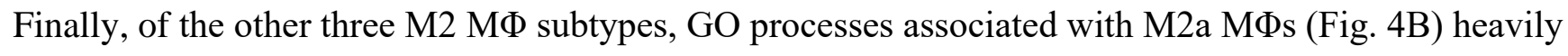
favored tissue-reparative, pro-fibrotic, pro-angiogenic, and phagocytic functions, while the upregulation of scavengers CD163 and MerTk (Supplemental Table 1) for M2c MФs are consistent with phagocytosis GO processes detected. Other M2c MФ identified GO processes included the Tie signaling pathway, known to play a key role in angiogenesis (Fig. 4D). Similarly, for M2d MФs nine of the top 17 GO processes were causally related to angiogenesis. Of the remaining eight listed processes, six contributed to ECM remodeling (Fig. 4E). In summary, both patterns of polarization detected through GSEA and DGS (Fig. 3) and direct comparison between phenotypes by GO annotation (Fig. 4) demonstrate unique and overlapping proposed biological 
functionality and further support the reorganization of M2b MФs from being a M2 subtype to being more closely related to M1 MФs in functionality.

\section{Global Metabolomics Demonstrates Functional MФ Phenotypes are also Distinct Metabotypes}

Global metabolomics profile of the parent, resting M0 MФs and five polarized МФ phenotypes identified 498 compounds of known identity. The top 50 metabolites, as determined by ANOVA analysis $(\mathrm{p}<0.05)$ and depicted in the heatmap (Supplemental Fig. 3G) demonstrated the immunomodulation of the individual phenotypes. The most striking distinction in the $\mathrm{HC}$ analysis is the metabolic differences, especially within lipid metabolism, observed between the M1 MФs, the M0 MФs and the M2 subtypes. Of specific interest is the clustering of the M2b MФs with the other M2 subtypes considering the M2b MФ propensity towards the M1 pro-inflammatory signature. The notable exception to this trend is the apparent similarities in M1 and M2b M $\Phi$ nicotinamide and tryptophan metabolism which is common to pro-inflammatory responses. Additionally, MФ metabotype clustering was demonstrated through OPLSDA and represented in the cross-validated score plots (inset plots in Fig. 5A.1 - E.1). Separation along the x-axis (between phenotype variation) in the cross-validated score plots quantifies metabotype cluster separation between each polarized MФ phenotype and the parent M0 MФs, with cluster separation greatest for M2b MФs (14.2\%) and M1 (13.0\%) and the least for M2d (10.3\%) and M2c (9.4\%) and 8.1\% MФs. The y-axis scores (orthogonal T score or within phenotype variation) reflected the notable variation observed within each MФ phenotype; however, the variation was similar for each phenotype $(\sim 60 \%)$. Furthermore, the larger within phenotype variance observed in this data warrants additional analysis and may indicate uncertainty in the metabotype prediction on the basis of cross-validation scores alone [43].

To identify putative metabolite biomarkers of high covariance and high correlation separating each polarized phenotype from the parent M0 MФ phenotype, OPLSDA model contribution from metabolite measurements (covariance; $\mathrm{x}$-axis) and the reliability of the measurements (correlation; $y$-axis) was projected in S-plots (Fig. 5A.1-E.1). Biomarkers with larger covariance $(-0.2 \leq$ covariance $\geq 0.2)$ and correlation $(-0.6 \leq$ 
correlation $\geq 0.6$ ) scores can identify biochemically significant metabolites with predictive value for metabolic modeling [43]. Normalized peak intensity for metabolites of interest as putative biomarkers that fall within these parameters are shown for each MФ phenotype (Fig. 5A.2-E.2), demonstrating the importance of metabolic fluidity to $\mathrm{M} \Phi$ polarization.

As the generally accepted paradigm is that MФ phenotypes fall into the classically activated M1 and alternatively activated M2, direct comparison of M1 MФ metabotype to each M2 MФ subtype was performed. To achieve this comparison, SUS graphs were employed to visualize OPLSDA characterization of metabolites impacted by polarization (Fig. 6A.1-D.1) and outcomes were projected as both shared and unique metabolite biomarkers between the two metabotypes, as well as associated correlation scores (Fig. 6A.2-D.2). Statistically significant metabolites $(\mathrm{FC}>2.0$ and $\mathrm{p}<0.05)$ are depicted in the associated volcano plots (Fig. 6A.3-D.3). Contrary to phenotyping by gene expression and correlated functionality, metabotyping each M $\Phi$ group did not result in clustering patterns with $\mathrm{M} 2 \mathrm{~b}$ MФs more closely related to M1 MФs and the rest of the M2 subtypes clustered together; however, the unique and shared metabolites within each comparison differed considerably, further indicating the unique metabolism of each phenotype.

Metabolites impacted by polarization and identified through metabotype comparison between M1 and M2b MФs (Fig. 5A,C and Fig. 6B) indicated metabolic investment in anti-microbial function with itaconate accumulation (correlation score $=0.91,0.64$, respectively) and antioxidant capacity evidenced by tryptophan consumption (correlation score $=-0.97,-0.91$, respectively), kynurenine accumulation (correlation score $=$ 0.93), and quinolinate accumulation (correlation score $=0.85,0.81$, respectively), as previously described [48]. Metabolites involved in glutamate metabolism such as $\mathrm{N}$-acetylglutamine (correlation score $=0.70,0.19$, respectively) correlated highly with M1 MФs only and branched-chain amino acid metabolites such as acetylcarnitine (correlation score $=-0.30,-0.83$, respectively) were uniquely correlated to M2b MФs.

As mentioned above, the remaining M2 MФ subtypes (M2a, M2c, and M2d) cluster separation from the parent M0 MФ indicated less metabolic divergence upon polarization (Fig. 5B, D, E); however, unique 
metabolic features could be distinguished between the subtypes. M2a MФs accumulated cysteine-GSSG and myo-inositol, metabolites involved with glutathione recycling and had similar correlation with uracil as M2b and M2c MФs, indicating a role for pyrimidine metabolism in these phenotypes (correlation score $=-0.61$, 0.66, -0.72, respectively) (Fig. 6A-C). Glutamate was consumed by both M2c and M2d MФs during polarization and correlation values were also prominent relative to M1 MФs (correlation score $=-0.58,-0.55$, respectively) (Fig. 5D-E and data not shown). Arginine catabolism was indicated by M2d M $\Phi$ accumulation of 2-oxoarginine (Fig. 5E.2) and for all M2 MФ subtypes as demonstrated by negative correlation scores for 4guanidinobutanoate in M2a, M2b, M2c, and M2d MФ (correlation score = $-0.87,-0.79,-0.61$, and -0.69, respectively) (Fig. 6A.2-D.2), while histidine metabolism was indicated for M2a and M2b MФs through negative correlation to imidazole lactate (correlation score $=-0.64,-0.68$, respectively) (Fig. 6A.2-B.2). Finally, notable differences in lipid metabolism between the M1 and M2 MФ phenotypes were exhibited by the accumulation of fatty acids and their derivatives in M1 MФs and corresponding negative correlations within the M2 MФ subtypes. For example, M2a MФ negative correlation with palmitoyl-myristoyl-glycerol (correlation score = -0.72), M2a and M2b MФ negative correlation with N-stearoyl-sphingosine (correlation score = -0.81, 0.70, respectively), M2c and M2d negative correlation with 1-stearoyl-2-oleoyl-GPS (correlation score = -0.64, -0.68, respectively), and M2d MФ negative correlation to 1-stearoyl-2-linoleoyl-GPS (correlation score = -0.63) (Fig. 6B.2-D.2; Supplemental Fig. 4B-F).

\section{Metabolic Pathway Analysis to Defined Unique Metabotypes for Each МФ Polarized Phenotype}

Using topological mapping of global metabolomics organized by p-value from pathway enrichment analysis (y-axis) relative to impact score from pathway topology analysis (x-axis), metabotype was further characterized for each polarized $\mathrm{M} \Phi$ phenotype and provided a global view of metabolic impact from polarization. For each node, color intensity and size illustrate p-value significance and pathway impact, respectively (Fig. 7). To further support metabotyping by global metabolomics pathway analysis, metabolism-related SDEGs up- or down-regulated relative to the resting parent M0 MФs are displayed (green or blue, respectively) (Fig. 8). 
Finally, a comprehensive summary of this metabolic analysis is illustrated in Figure 9. Each colored box above the listed metabolite represents significant fold change relative to the parent M0 MФs $\left(1<\log _{2}<-1\right)$. Numerical values corresponding to the color scale are included in Supplemental Table 2.

As MФs are well characterized to be glycolytic, it was not surprising that $F B P 1$, the gene encoding the FBPI regulator of gluconeogenesis, was significantly down regulated in all the polarized MФs, especially M1 and M2b MФs (Fig. 8A). Additionally, the accumulation of glucose observed in the M2c and M2d MФs (Fig. 9: Glycolysis \& Gluconeogenesis) suggests phenotypic variation in the utilization of glucose in $\mathrm{M} \Phi$ immunometabolism. The accumulation of glycerate (Fig. 9: PPP) in the M1, M2b and M2d MФs is suggestive of flux into the PPP for each of these phenotypes. From the topography analysis, the most statistically significant pathway observed was pyrimidine metabolism for both M1 and M2b MФs (Fig. 7A \& C; Fig. 9) while the purine pathway was most prevalent for M2d (Fig. 7E; Fig. 9). Furthermore, the upregulation of thioredoxin (encoded by $T X N$ ), a redox mediator, in M1 MФs and the CD44 transcript in M1 and M2b MФs (Fig. 8A and 8D), known to enhance glycolysis and metabolic flux into the PPP [49], reinforced this pathway for immunometabolism in M1 and M2b MФs.

One established metabotype feature of M1 polarization is the decoupled TCA after citrate and succinate, marked by accumulation of itaconate and succinate, which was observed in the M1 polarized MФs (Fig. 9). Moreover, the upregulation of genes encoding an itaconate regulator, IRG1 (Fig. 8A), and succinate-stabilized HIF-1 a transcript (Supplemental Table 1) was consistent with these findings. Interestingly, M2b MФs also exhibited similar IRGI and HIF-1 $a$ upregulation (Fig. 8A) along with itaconate accumulation; however, these

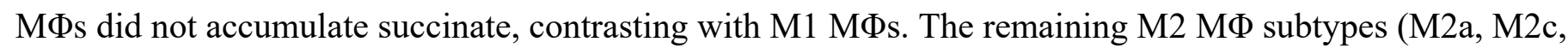
and M2d) all demonstrated metabolic flux patterns consistent with an intact TCA (Fig. 7-9).

For amino acid metabolism, the Arg \& Pro and Ala, Asp, \& Glu metabolic pathways were similarly

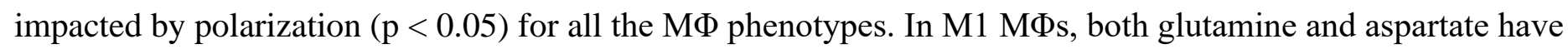
been shown to replenish TCA metabolites such as $\alpha$-ketoglutarate and fumarate, presumably depleted as a result 
of a decoupled TCA [28], and steady-state levels of both fumarate and malate were observed in the M1 MФ, as well as accumulation of glutamine to feed into the TCA (Fig. 9). While only M1 MФs accumulated alanine and asparagine, aspartate was accumulated in all polarized phenotypes apart from M2d MФs (Fig.9). Aspartate accumulation can drive $\beta$-alanine metabolism, which was a highly impacted metabolic pathway for the M1, M2a, M2b, and M2d MФs (Fig. 7A, B, C, and E) and was consumed within these phenotypes during polarization (Supplemental Table 2 and Fig. 9: Pyrimidine). Aspartate can also lead to quinolinate production, as observed for M1 and M2b MФs (Fig. 9: Nicotinate \& Nicotinamide). In conjunction with upregulation of CD38, IDO1, and NAMPT (Fig. 8A \& B), consumption of tryptophan, and accumulation of kynurenine (Fig. 9), these results reaffirmed previously findings highlighting the importance of tryptophan and nicotinamide metabolism within the M1 and M2b MФs. In all M2 MФ subtypes, taurine metabolism was impacted to various degrees during polarization, while cysteine and methionine metabolism were impacted by polarization in $\mathrm{M} 2 \mathrm{~b}$, M2c, and M2d MФ (Fig. 7B - E). Cysteine, taurine, and hypotaurine were generally consumed for the M2 M $\Phi$ subtypes (Fig. 9), with cysteine potentially feeding directly into glutathione metabolism in response to oxidative stress (Fig. 9). Moreover, diminished levels of oxidized glutathione and $\gamma$-glutamylamino acids, produced as a part of the $\gamma$-glutamyl cycle, further indicate oxidative stress associated with polarization across phenotypes (Fig. 9 and Supplemental Fig. 4A). Finally, Gly, Ser, and Thr metabolism was only identified as significant within the M1 MФs (Fig. 7A), supporting previous findings in LPS-stimulated MФs within mice [50].

Metabotyping in all five polarized MФ phenotypes identified arginine metabolism as both impacted and significant (Fig. 7A-E). While intracellular stores of arginine did not appear to change with polarization, significant changes to metabolites upstream and downstream of arginine did, including arginosuccinate which feeds into the TCA Cycle, 1-pyrroline-5-carboxylate which derives from the $\gamma$-glutamyl cycle, and ornithine and citrulline of the Arg-Cit Cycle (Fig. 9: Urea Cycle). Interestingly, despite extensive literature around the expression of arginase (Arg1) as a marker of alternatively activated M2 MФs, in our model expression of this gene was not significantly upregulated relative to the parent M0 MФ phenotype (Fig. 8B); however, 


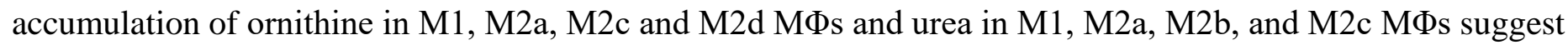
this enzyme is activated upon polarization (Fig. 9). Downstream of 1-pyrroline-5-carboxylate, proline and hydroxyproline accumulated in M1 MФs, suggesting that the remaining M2 MФ subtypes may utilize these metabolites for collagen biosynthesis (Fig. 9) [51]. Finally, arginine can be diverted to synthesize creatine through guanidinoacetate, which is highly consumed in both the M1 and M2b MФs upon polarization (Fig. 9) and has been shown to suppress IFN- $\gamma$ responses while facilitating IL-4 polarization in MФs [52].

Other pathways of interest impacted by polarization included pyrimidine and purine metabolism (Fig. 7AE). In general, these metabolic pathways were highly consumed across all MФ phenotypes, but especially notable in the M1 and M2b MФ consumption of dioxy- and trioxy-pyrimidines (Fig. 9). An offshoot of pyrimidine metabolism through $\beta$-alanine, polyamine metabolism was also markedly impacted with consumption of spermidine and accumulation of N1-acetyl-spermidine in both M1 and M2b MФs upon polarization (Fig. 9). Hexosamine metabolism displayed a similar pattern with regards to M1 and M2b M $\Phi$ consumption; however, all three other M2 MФ subtypes displayed metabolite accumulation, specifically of UDP-glucose, UDP-galactose, and UDP-glucuronate (Fig. 9). Whether these metabolites drive tissue regeneration/repair or act as signaling molecules within each MФ phenotype, remains to be determined.

Finally, lipid metabolism was impacted in all five polarized MФs, especially glycerophospholipid and sphingolipid metabolism, as shown by significance of impact (Fig. 7A-E). COX-2 (PTGS2) transcript, encoding the enzyme involved in conversion of arachidonic acid to the pro-inflammatory precursor prostaglandin $\mathrm{H} 2$, was upregulated in M1 and M2b MФs, while the transcript ALOX5, associated with anti-inflammatory response, were downregulated in M1 MФs (Fig. 8D). Of the other alternatively activated M2 MФ phenotypes, M2a MФs upregulated $A L O X 15$, which facilities activation of known $\mathrm{M} 2 \mathrm{a} \mathrm{M} \Phi$ regulators (PPARG, $M R C 1$, and $C D 36$ )

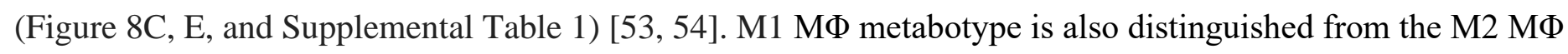
subtypes by FA metabolism, with transcripts $C D 36, E N P P 2, F A S N$, and $L P L$ all downregulated with polarization and upregulated in M2a, M2c, and M2d to various levels (Fig. 8C). Lipid metabolite profiles 
reflected this distinction for the M1 MФ metabotype, with general patterns of lipid accumulation for M1 MФs and consumption of lipids for the M2 MФ metabotypes, with the exception of DHA known to have antiinflammatory properties (Supplemental Fig. 4B-F) [55]. Interestingly, the most dominant MФ phenotype with regard to lipid metabolism was the $\mathrm{M} 2 \mathrm{~b} \mathrm{M} \Phi$ within which the levels of metabolite consumption upon polarization generally dwarfed all other phenotypes and, in contrast to similar myeloid functionality profiles, was clearly different from the M1 MФ phenotype (Supplemental Fig. 4B-F). What impact this difference in lipid metabolism has on biological systems is under active investigation.

\section{МФ Effector Functions and Metabotype Dynamics Over Time}

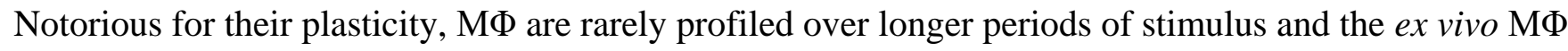
polarization model presented herein provided an excellent platform to compare polarization impact at different timepoints. To evaluate temporal shifts in both $M \Phi$ effector function and metabotype, polarized $M \Phi$ were harvested at both 24- and 72-hours polarization. Within the inflammatory protein subgroup, TNF $\alpha$, CCL3, and CXCL8 exhibited minor significant increase only in TNFa from M1 MФs at 72 hours polarization (Fig. 10A). While M1 MФs markedly increased IL-12p70 and decreased IP-10 production between the two time points, IFN $\alpha$ production was decreased for M1, M2b, and M2c M $\Phi$ phenotypes (Fig. 10A). Among the inflammation regulating/tissue repair proteins (Fig. 10B) and growth factors (Fig. 10C), IL-10 and IL-6 production was significantly decreased from 24 to 72 hours polarization for both the M1 and M2b M $\Phi$ phenotypes.

Interestingly, IL-1 $\beta$ concentrations increased dramatically for both phenotypes, while TGF- $\beta$ concentrations decreased for all polarized MФ phenotypes (Fig. 10B).

Additionally, GO analysis demonstrated differentially expressed biological pathways between the 24- and 72-hour time points for all polarized phenotypes. M1 MФs displayed a strong inflammatory signature at 24 hours, which was sustained at 72 hours (Fig. 11A, light red bars); however, by 72 hours, the M1 MФs had initiated cellular protective/death processes such as the prostaglandin-endoperoxide synthase, negative regulation of apoptosis, and programmed necrotic cell death GO processes (Fig. 11A, dark red bars). For the 
M2a MФs, GO processes at 24 hours heavily favored cellular proliferation, ECM remodeling, and angiogenesis

(Fig. 11B, light yellow bars). While these cells still exhibited a potential for angiogenesis and ECM remodeling at 72 hours, the upregulation of the cyclooxygenase, leukotriene, histamine, and IFN- $\alpha$ processes at 72 hours were consistent with activation of anti-inflammatory function, viral inhibition, and the pathogenesis of asthma (Fig. 11B, dark yellow bar) [56]. As discussed above, M2b MФs share a strong inflammatory functional signature with M1 MФs at 24 hours (Fig. 11C, light green bars); however, by 72 hours, the M2b MФs had upregulated genetic programs primarily associated with metabolism (Fig. 11C, dark green bars). ECM remodeling, angiogenesis, and efferocytosis functionality associated with M2c MФs remained relatively consistent across both time points, with the interesting exception of IL-23 and IL-18 regulation, which are both generally associated with pro-inflammatory functionality. Finally, M2d M $\Phi$ functionality at 72 hours persisted with the angiogenic, profibrotic, and anti-inflammatory potential (Fig. 11D, light and dark purple bars); however, lipid metabolism was uniquely profiled at 72 hours, raising the interesting question of how this might translate to immunomodulation in this M2 MФ subtype.

As described above, the top 15 impacted metabolic pathway scores were utilized to generate radar plots comparing 24- and 72-hour metabotypes in all five polarized MФs (Fig. 12A-E, hatched and solid graphs for 24 and 72 hours, respectively). All phenotypes demonstrated significant shifts in metabolism from 24- to 72-hours polarization. For M1, M2a, M2b, and M2c MФs, glycolysis and the PPP remained a significant part of the metabotype profile at 72 hours; however, glycolysis was significantly increased in the M2a MФs (Fig. 11B), while remaining consistent in all of the other phenotypes. PPP metabolism also increased in the M1, M2a and M2b MФs, but decreased in M2c MФs. Additionally, amino acid and lipid metabolism was significant enhancement for both the M1 and M2b MФs at 72 hours. The global differences in metabolic impact between the M2a (Fig. 11B) and M2c (Fig. 11D) MФs is quite interesting in that they demonstrated nearly opposite metabotype profiles for the two time points. For example, M2a MФs exhibited higher impact scores for several amino acids, including BCAA, as well as sphingolipid, galactose, glutathione, and purine metabolism at 24 
hours (Fig. 11B, hatched plot), whereas these same pathways were more highly impacted at 72 hours for M2c

MФs (Fig. 11D, solid plot). Another interesting comparison is the different shifts in galactose, glutathione, purine, and pyrimidine metabolism in the M1 and M2b MФs (Fig. 11A \& C), further supporting the distinct metabotype profiles of these phenotypes despite their similar inflammatory signatures. Finally, in contrast to M1, M2a, and M2b MФ metabolic dynamics, from 24 hrs. to 72 hours., M2d MФs undergo a significant constriction in metabolic activity (Fig. 11E). This is especially evident when compared to the M2b MФs, which significantly expand metabolic activity at 72 hours (Fig. 11C). While these observations in the dynamics of M $\Phi$ functionality and metabotype over time demonstrate that each of these $M \Phi$ phenotypes can exhibit significant plasticity, the causal relationship between metabolism and biological function remains an expansive area for future investigations.

\section{Discussion}

Previous studies have described multiple MФ phenotypes; however, to our knowledge, this study represents the most comprehensive profile and complete integration of metabotype within the known functional phenotypes. $\mathrm{M} \Phi$ plasticity is uniquely dynamic and the integration of metabotype and functional phenotype presented herein provides a framework for further investigation into how this dynamic plasticity is driven.

Furthermore, understanding how global metabolic shifts contribute to MФ plasticity provides for greater insight into mechanisms of wound healing and provides the foundation for novel innovations in therapeutic design.

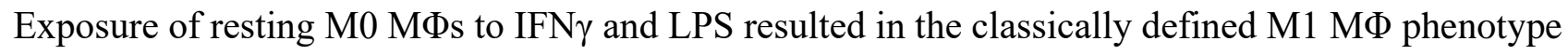
characterized by a strong, proinflammatory signature as evidenced by the secretion of several inflammatory cytokines and chemokines (Fig. 2A), the upregulation of M1-associated transcripts (Supplemental Table 1), and the biological functional profile identified through GO process analysis (Fig. 4). Furthermore, distinct metabolic shifts essential to this M1 MФ functionality were also identified. Analysis of our metabolic data suggest reprogramming from OXPHOS to aerobic glycolysis, necessary for rapid ATP production and the provision of 
several glycolytic intermediates such as PKM2, GAPDH, and enolase for IL- $1 \alpha / \beta$, IL-6, and TNF $\alpha$ production (Fig. 2 \& 9) [31, 57-59]. The diversion of glycolytic intermediates into the PPP drives the production of nucleotides, secondary metabolic intermediates, and the nucleotide substrate, NADPH [10, 28].

In parallel, pyruvate from glycolysis feeds into the observed decoupled TCA cycle. The first break before isocitrate results in an accumulation of citrate, which is diverted for itaconate production and de novo FAS. Upon LPS stimulation, the upregulation of IRGl (Fig. 8 and Supplemental Table 1) encodes the enzyme necessary for itaconate production (Fig. 5A, 9 and Supplemental Table 2) [60]. In addition to antimicrobial activity [61], itaconate serves as an inhibitor of SDH resulting in the accumulation of succinate (Fig. 9 and Supplemental Table 2) and the second break in the TCA cycle by M1 MФs [60,61]. The role of succinate as an inflammatory signal in MФs is complex. Succinate oxidation through SDH together with an increased mitochondrial potential result in an increase of mitochondrial ROS production. Furthermore, accumulated succinate together with succinate recycling through GPR91 generates a feedforward loop that enhances HIF-1 $\alpha$ stabilization (Supplemental Table 1) and the production of IL-1 $\beta$ (Fig. 2B) [27, 61, 62]. Additionally, excess citrate, once exported into the cytosol, can be converted into acetyl-CoA, oxidized to form malonyl-CoA, and converted into nascent FA products through the NADPH-dependent FASN [10]. Within the context of M1 polarization, the observed lipid accumulation (Supplemental Fig. 4) is essential for the synthesis of inflammatory mediators [63], prostaglandin synthesis through arachidonic metabolism (Fig. 8D) [64], and the membrane biosynthesis necessary for the phagocytic potential of these cells (Fig. 1) [65].

For the M2a MФs, our metabolic results support both active glycolysis and an intact TCA cycle (Fig. 9 and Supplemental Table 2) although glucose does not appear to be consumed at the same rate as it was in the M1 phenotype. While M2a MФs demonstrate active glycolysis, which is used to supply the TCA cycle, M2a polarization has been shown to be unaffected during glucose deprivation. This observation suggests that other metabolic pathways, such as glutaminolysis, can also be utilized to support the TCA cycle and drive oxidative metabolism [65]. We observed decreased pools of lipids in this МФ subtype (Supplemental Fig. 4), suggesting 
that FAO is indeed active. Previous research has shown that glucose oxidation and mitochondrial respiration are necessary for early IL-4 polarization, but not FAO; however, FAO, dependent on CD36 expression (Fig. 8C) [32], was established as critical for M2a functionality at later time points [33]. Furthermore, suppression of CD36 FA uptake along with FAO has been shown to ameliorate M2a polarization during parasitic helminth infections [32]. Using metabolic flux analysis and glutamine deprivation, Jha, et al., (2015) demonstrated the necessity of glutamine for amino and nucleotide sugar metabolism and M2a polarization markers CD301 and MRC1 [28]. While glutamine flux analysis is outside of the scope of the research presented herein, we did observe increased metabolic activity within the hexosamine biosynthesis pathway and increased pools of UDPGlcNAc-associated modules (Fig. 9 and Supplemental Table 2).

Much of what is known of the biologically functionality of the M2a phenotype lies within the context of wound healing. In the late stages of inflammation, M2a MФs facilitate the clearance of glycosylated pathogens through the upregulated cell-surface MRC1 (Supplemental Table 1) and the efferocytosis of apoptotic cells in a CD36-dependent fashion [66]. Additionally, MRC1 is known to target mannosylated antigen intracellularly for MHC-II compartments and antigen presentation in dendritic cells [67]. Interestingly, our M2a polarized MФs show increased expression of the MHC-II receptor HLA-DR (Fig. 1D \& H) suggesting a similar role for this MФ subtype. In addition to pathogen clearance, the MRC1 removes glycosylated inflammatory proteins from the wound microenvironment [68]. M2a MФs also upregulate and secrete numerous biological factors, such as IL-1RA (Supplemental Fig. 1G) to suppress IL-1 mediated inflammation [17], the chemokine ligands CCL3 (Fig. 2A), CCL17, CCL18, and CCL22 (Supplemental Table 1), and growth factors GM-CSF, TGF- $\beta$, and PDGF (Fig. 2B \& C) needed to promote the recruitment and differentiation of numerous cell types necessary for the proliferative phase of wound healing [69].

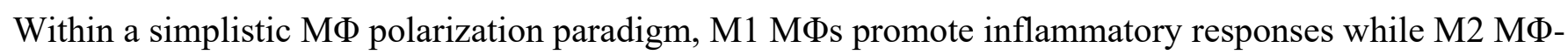
associated phenotypes drive anti-inflammatory, wound healing functions; however, resting MФs exposed to TLR-agonist, endogenous ligands coupled with FC $\gamma$ receptor ligation and signaling through SYK resulted in a 
polarized phenotype that defies the M1/M2 paradigm by secreting strong pro-inflammatory cytokines while promoting biological effector mechanisms associated with the M2 family of MФs [20, 70]. Indeed, Vogelpoel, et al. (2014) demonstrated that TLR agonists alone, using both LPS and Pam3CSK4, could not produce this phenotype that is commonly known as M2b MФs [8]. Using polarization stimulus through LPS and IC, this M $\Phi$ phenotype was demonstrated to secrete proinflammatory cytokines TNF $\alpha$, IL-1 $\beta$, and IL-6 (Fig. 2A \& B) and upregulated transcripts, including $T N F, I L-1 \beta, I L-23 A, I L-10, C C L 1$, and CCL5 (Supplemental Table 1), that promote Th17 responses [9] and profile as distinct from any other M2 MФ subtype.

Metabolically, M2b MФs displayed similar glycolysis, tryptophan, amino sugar, and pyrimidine metabolism

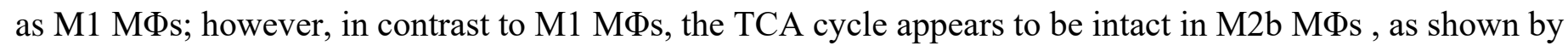
the lack of succinate accumulation (Fig. 9). In LPS-activated MФ, HIF-1 $\alpha$ stabilization and IL-1 $\beta$ production has been linked to succinate accumulation via glutamine-dependent anaplerosis or the $\gamma$-aminobutyric acid (GABA) shunt [27]; however, this does not appear to be an active mechanism in M2b MФs and thus warrants further investigation. Additionally, the M2b MФ lipid metabolite profile suggests active FAO or OXPHOS similar to the other M2 subtypes (Supplemental Fig. 4). M2b MФs demonstrate complex functionality. For example, the inflammatory signature can be readily seen contributing to the pathology of chronic inflammatory conditions such as rheumatoid arthritis [8] and SLE [9]; however, M2b MФs have also been associated with aberrant antimicrobial responses [21, 71], reduced cardiac fibrosis after ischemia/reperfusion injuries [72], and improvement of intestinal colitis [73].

IL-10, the polarizing stimuli for M2c MФs in this study, is a known inducer of CD163 expression that has historically defined this functional phenotype [23]; therefore, it was not surprising that cell-surface marker analysis characterized this M2 MФ subtype as $\mathrm{CD} 86^{\text {low }} \mathrm{CD} 163^{\text {high }}$ (Fig. 1). These findings, along with the observed upregulation of MerTk (Supplemental Table 1), a key component of efferocytosis, is consistent with phagocytosis GO processes being profiled (Fig. 4). CD163, the hemoglobin scavenger receptor, mediates Hboxidative tissue damage following hemolysis and induces the expression of the anti-inflammatory enzyme heme 
oxidase 1 (HMOX1; data not shown) [74]. Additionally, CD163 expression accompanied by other antiinflammatory mediators was observed in cardiac bypass patients post-surgery and in volunteers recovering from cantharidin-induced skin blisters [75]. Our findings are in line with these studies and are consistent with a functional phenotype responsible for the regulation and mediation of inflammation, vascular insult, and oxidative stress $[15,74,75]$.

To date, extraordinarily little is known regarding the metabolism of M2c MФs and has been limited to carbohydrate and glutamate metabolism. Rodriguez-Prados, et al. (2010) concluded that M1, M2a, and M2c polarized MФs were glycolytic cells that converted up to $95 \%$ of glucose into lactate. While this conversion was accelerated for the M1 cells, both M2a and M2c MФs exhibited basal glucose consumption levels comparable to the M0 parent cells [30]. Interestingly, our results demonstrated that M2c MФs accumulated a significant amount of glucose relative to the M0 MФs (Fig. 9 \& Supplemental Table 2), indicating either a slower glycolytic capacity or the ability the import glucose at higher concentrations. Additionally, these authors found differences in glutamine consumption $(\mathrm{M} 1>>\mathrm{M} 2 \mathrm{a}>>\mathrm{M} 0>\mathrm{M} 2 \mathrm{c})$ and glutamate production $(\mathrm{M} 1>\mathrm{M} 2 \mathrm{c}>\mathrm{M} 2 \mathrm{a}>\mathrm{M} 0)$ in their study [30]. While we observed different accumulations of glutamine and glutamate in these phenotypes, the M2c MФs displayed considerably diminished pools of glutamate in comparison to the M0 parent cells (Fig. 9 \& Supplemental Table 2). Given the necessity of glutamate in ECM remodeling, it is probable that glutamate is being diverted to collagen synthesis (as reviewed in Karna, et al. 2020 [76]). In addition to active glycolysis, an observed shift to the PPP via glucuronate and the accumulation of UDP-glucuronate (Fig. 9 and Supplemental Table 2) suggests a potential investment in the synthesis of hyaluronic acid for tissue regeneration and anti-inflammatory responses during wound healing [77]. M2c MФs also demonstrated an intact TCA cycle, enhanced OXPHOS, and lipid catabolism (data not shown and Supplemental Fig. 4). There also appears to be concurrent urea and aspartate-arginosuccinate cycles (Fig. 9) as well as investment in BCAA metabolism (Fig. 9). 
Given the remarkable plasticity of MФs and the equally disparate array of tumor-specific

microenvironments, it is not surprising that TAMs, as a collective, exhibit diverse functionality resembling both M1 and M2 polarized phenotypes [78]. As such, TAMs have been associated with protein expression patterns observed in both acute wounding (i.e. IL-6, and IL-1 $\beta$ ) and wound resolution (i.e. TGF- $\beta$, PDGF and VEGFA) [26]. IL-6 appears to be central to many of the underlying processes that drive TAM functionality. Muliaditan, et al. (2018) demonstrated that TAM-produced IL-6 regulates the expression of HMOX1 which has been associated with poor overall survival rates. In our IL-6 polarized M2d MФs, we also observe the upregulation of HMOX1 (data not shown), supporting these findings [26]. In terms of overall biological functionality, TAMs have been shown to facilitate angiogenesis [79], tumor progression [6], immunosuppression [25], and therapeutic resistance [5]. Our transcriptional profile of these MФs directly supports all these functions (Fig. 4). Furthermore, TGF- $\beta$ and PDGF-BB (Fig. 2C), secreted at significant levels in these cells, are well-documented factors associated with tumor progression and generally poor outcomes [80].

The tumor metabolic environment typically has some degree of hypoxia, which promotes TAM functional plasticity in support of tumor survival. This hypoxia shifts TAMs towards oxidative metabolism and promotion of neoangiogenesis and metastasis [79]. In our model, M2d MФs were not cultured under hypoxic conditions and glucose accumulation was observed (Fig. 9), indicating functional glycolysis, as has been observed in primary tumors $[4,81,82]$. The tumor environment has also been characterized as abundant in lipids and M2d MФs were observed to highly express CD36, the fatty acid translocase integral membrane protein (Fig. 7). Interestingly, in a murine melanoma model, CD36 expression on TAMs was found to mediate uptake of oxidized LDL and promote tumorigenesis [83]. FAO utilization in TAMS has also been proposed to be mediated through epigenetic reprogramming and pharmacological inhibition of FAO favors M2-to-M1 repolarization in murine cancer models [25, 84]. In our model system, the M2d MФs displayed significant FAO, indicating a key role for this functional phenotype (Fig S8); however, investigation into potential epigenetic modifications cellular cross-talk with tumorigenic cells is outside of the scope of the present study. 
Finally, macrophage functional plasticity and immunomodulatory effects of metabolism have rarely been observed over an extended period. By extending our ex vivo MФ polarization model out to 72 hours post polarization, the temporal effects of stimulus were observed. While pro-inflammatory MФs (M1) shifted to programmed cell death and anti-inflammatory MФs (M2a and M2c) continued to promote tissue remodeling/repair function as would be expected with extended stimulus, M2b and M2d MФs demonstrated significant and opposite shifts in metabolic programming. For example, apart from glycerophospholipid metabolism and BCAA degradation, the M2d MФs displayed a massive contraction of metabolic activity, while the M2b MФs display a massive increase in metabolic activity (Fig. 12C, E). The functional consequences of this metabolic shift are suggested by down regulation of TGF $\beta$ in M2d MФs and up regulation of IL-1 $\beta$ in M2b MФs; however, further investigation is warranted and ongoing.

In conclusion, this study presents the most comprehensive functional phenotyping of MФ polarization plasticity and associated metabotypes to date. While the ex vivo MФ model system utilized primary, human blood derived MФs and allowed homogeneous polarization of $\mathrm{M} \Phi$ phenotypes, the functional phenotypes and associated metabotypes presented herein must be established in vivo and in functional context. In situ tissue profiling within primary, human wound tissue is ongoing; however, demonstrating the potential in vivo context across numerous tissues and pathologies provides unlimited opportunity for investigation. In addition, the work presented herein demonstrates correlation between MФ functional phenotype and metabotype but deciphering the intricate interplay between metabolism and immunomodulation at the heart of causation is outside of the scope of the present investigation. Clearly, next steps must include contextualizing these profiles and teasing out the mechanistic structure of metabolic immunomodulation of $М \Phi$ functional plasticity.

\section{Authorship}

C.B.A, M.C.B.A, and M.M.D. conceptualized and designed the experimental approach and supervised sample collection for this research. C.B.A, T.M.W.L., H.L.S., and J.G. contributed equally to the execution of the 
experiments and data collection. C.B.A and M.C.B.A. performed the data analysis, generated the figures, and wrote the manuscript. All authors participated in the editing process.

\section{Acknowledgments}

This work was supported in part by the U.S. Department of Veterans Affairs, Office of Research and

Development Biomedical Laboratory Research Program, the Idaho INBRE Program (NIH NIGMS P20

GM103408), a NIAID-NIH award (RO3AI135998; PI Ammons), and the IVREF Center of Biomedical

Research Excellence in Emerging/Reemerging Infectious Diseases (NIH NIGMS P20GM109007). This content is solely the responsibility of the authors and does not necessarily represent the official views of the National Institutes of Health, U.S. Department of Veterans Affairs, or the United States Government.

\section{Disclosures}

The authors declare no conflicts of interest.

\section{References}

1. Kodelja, V., Müller, C., Tenorio, S., Schebesch, C., Orfanos, C. E., Goerdt, S. Differences in angiogenic potential of classically vs alternatively activated macrophages. Immunobiology 1997; 197: 478-493.

2. Stout, R. D., Jiang, C., Matta, B., Tietzel, I., Watkins, S. K., Suttles, J. Macrophages sequentially change their functional phenotype in response to changes in microenvironmental influences. The Journal of Immunology 2005; 175: 342-349.

3. Daley, J. M., Brancato, S. K., Thomay, A. A., Reichner, J. S., Albina, J. E. The phenotype of murine wound macrophages. Journal of Leukocyte Biology 2010; 87: 59-67.

4. Penny, H. L., Sieow, J. L., Adriani, G., Yeap, W. H., See Chi Ee, P., San Luis, B., Lee, B., Lee, T., Mak, S. Y., Ho, Y. S. Warburg metabolism in tumor-conditioned macrophages promotes metastasis in human pancreatic ductal adenocarcinoma. Oncoimmunology 2016; 5: e1191731.

5. Halbrook, C. J., Pontious, C., Kovalenko, I., Lapienyte, L., Dreyer, S., Lee, H.-J., Thurston, G., Zhang, Y., Lazarus, J., Sajjakulnukit, P. Macrophage-released pyrimidines inhibit gemcitabine therapy in pancreatic cancer. Cell metabolism 2019; 29: 1390-1399. e6. 
6. Cao, W., Peters, J. H., Nieman, D., Sharma, M., Watson, T., Yu, J. Macrophage subtype predicts lymph node metastasis in oesophageal adenocarcinoma and promotes cancer cell invasion in vitro. British journal of cancer 2015; 113: 738-746.

7. Gibbings, S. L., Goyal, R., Desch, A. N., Leach, S. M., Prabagar, M., Atif, S. M., Bratton, D. L., Janssen, W., Jakubzick, C. V. Transcriptome analysis highlights the conserved difference between embryonic and postnatal-derived alveolar macrophages. Blood 2015; 126: 1357-1366.

8. Vogelpoel, L. T. C., Hansen, I. S., Rispens, T., Muller, F. J. M., van Capel, T. M. M., Turina, M. C., Vos, J. B., Baeten, D. L. P., Kapsenberg, M. L., de Jong, E. C., den Dunnen, J. Fc gamma receptor-TLR cross-talk elicits pro-inflammatory cytokine production by human M2 macrophages. Nature communications 2014; 5: 5444-5444.

9. Tsanaktsi, A., Solomou, E. E., Liossis, S.-N. C. Th1/17 cells, a subset of Th17 cells, are expanded in patients with active systemic lupus erythematosus. Clinical Immunology 2018; 195: 101-106.

10. O'Neill, L. A. J., Kishton, R. J., Rathmell, J. A guide to immunometabolism for immunologists. Nat Rev Immunol 2016; 16: 553-565.

11. Lachmandas, E., Boutens, L., Ratter, J. M., Hijmans, A., Hooiveld, G. J., Joosten, L. A., Rodenburg, R. J., Fransen, J. A., Houtkooper, R. H., Van Crevel, R. Microbial stimulation of different Toll-like receptor signalling pathways induces diverse metabolic programmes in human monocytes. Nature microbiology 2017; 2: 16246.

12. Krausgruber, T., Blazek, K., Smallie, T., Alzabin, S., Lockstone, H., Sahgal, N., Hussell, T., Feldmann, M., Udalova, I. A. IRF5 promotes inflammatory macrophage polarization and $\mathrm{T}_{\mathrm{H}} 1-\mathrm{T}_{\mathrm{H}} 17$ responses. Nature immunology 2011; 12: 231.

13. Rak, G. D., Osborne, L. C., Siracusa, M. C., Kim, B. S., Wang, K., Bayat, A., Artis, D., Volk, S. W. IL33-Dependent Group 2 Innate Lymphoid Cells Promote Cutaneous Wound Healing. Journal of Investigative Dermatology 2016; 136: 487-496.

14. Edwards, J. P., Zhang, X., Frauwirth, K. A., Mosser, D. M. Biochemical and functional characterization of three activated macrophage populations. Journal of Leukocyte Biology 2006; 80: 1298-1307.

15. Lurier, E. B., Dalton, D., Dampier, W., Raman, P., Nassiri, S., Ferraro, N. M., Rajagopalan, R., Sarmady, M., Spiller, K. L. Transcriptome analysis of IL-10-stimulated (M2c) macrophages by nextgeneration sequencing. Immunobiology 2017; 222: 847-856.

16. Duluc, D., Delneste, Y., Tan, F., Moles, M.-P., Grimaud, L., Lenoir, J., Preisser, L., Anegon, I., Catala, L., Ifrah, N., Descamps, P., Gamelin, E., Gascan, H., Hebbar, M., Jeannin, P. Tumor-associated leukemia inhibitory factor and IL-6 skew monocyte differentiation into tumor-associated macrophagelike cells. Blood 2007; 110: 4319-4330.

17. Fenton, M. J., Buras, J., Donnelly, R. IL-4 reciprocally regulates IL-1 and IL-1 receptor antagonist expression in human monocytes. The Journal of Immunology 1992; 149: 1283-1288.

18. Jetten, N., Verbruggen, S., Gijbels, M. J., Post, M. J., De Winther, M. P. J., Donners, M. M. P. C. Antiinflammatory M2, but not pro-inflammatory M1 macrophages promote angiogenesis in vivo. Angiogenesis 2014; 17: 109-118.

19. Gerber, J. S. and Mosser, D. M. Reversing lipopolysaccharide toxicity by ligating the macrophage Fc $\gamma$ receptors. The Journal of Immunology 2001; 166: 6861-6868.

20. Ambarus, C. A., Santegoets, K. C. M., van Bon, L., Wenink, M. H., Tak, P. P., Radstake, T. R. D. J., Baeten, D. L. P. Soluble immune complexes shift the TLR-induced cytokine production of distinct polarized human macrophage subsets towards IL-10. PloS one 2012; 7: e35994-e35994.

21. Nishiguchi, T., Ito, I., Lee, J. O., Suzuki, S., Suzuki, F., Kobayashi, M. Macrophage polarization and MRSA infection in burned mice. Immunology and cell biology 2017; 95: 198-206.

22. Novak, M. L. and Koh, T. J. Macrophage phenotypes during tissue repair. Journal of leukocyte biology 2013; 93: 875-881. 
23. Ohlsson, S. M., Linge, C. P., Gullstrand, B., Lood, C., Johansson, Å., Ohlsson, S., Lundqvist, A., Bengtsson, A. A., Carlsson, F., Hellmark, T. Serum from patients with systemic vasculitis induces alternatively activated macrophage M2c polarization. Clinical Immunology 2014; 152: 10-19.

24. Gabriel, V. A., McClellan, E. A., Scheuermann, R. H. Response of human skin to esthetic scarification. Burns 2014; 40: 1338-1344.

25. Hossain, F., Al-Khami, A. A., Wyczechowska, D., Hernandez, C., Zheng, L., Reiss, K., Del Valle, L., Trillo-Tinoco, J., Maj, T., Zou, W. Inhibition of fatty acid oxidation modulates immunosuppressive functions of myeloid-derived suppressor cells and enhances cancer therapies. Cancer immunology research 2015; 3: 1236-1247.

26. Muliaditan, T., Caron, J., Okesola, M., Opzoomer, J. W., Kosti, P., Georgouli, M., Gordon, P., Lall, S., Kuzeva, D. M., Pedro, L. Macrophages are exploited from an innate wound healing response to facilitate cancer metastasis. Nature communications 2018; 9: 1-15.

27. Tannahill, G. M., Curtis, A. M., Adamik, J., Palsson-McDermott, E. M., McGettrick, A. F., Goel, G., Frezza, C., Bernard, N. J., Kelly, B., Foley, N. H., Zheng, L., Gardet, A., Tong, Z., Jany, S. S., Corr, S. C., Haneklaus, M., Caffrey, B. E., Pierce, K., Walmsley, S., Beasley, F. C., Cummins, E., Nizet, V., Whyte, M., Taylor, C. T., Lin, H., Masters, S. L., Gottlieb, E., Kelly, V. P., Clish, C., Auron, P. E., Xavier, R. J., O'Neill, L. A. J. Succinate is an inflammatory signal that induces IL-1 $\beta$ through HIF- $1 \alpha$. Nature 2013; 496: 238-242.

28. Jha, Abhishek K., Huang, Stanley C.-C., Sergushichev, A., Lampropoulou, V., Ivanova, Y., Loginicheva, E., Chmielewski, K., Stewart, Kelly M., Ashall, J., Everts, B., Pearce, Edward J., Driggers, Edward M., Artyomov, Maxim N. Network Integration of Parallel Metabolic and Transcriptional Data Reveals Metabolic Modules that Regulate Macrophage Polarization. Immunity 2015; 42: 419-430.

29. Michelucci, A., Cordes, T., Ghelfi, J., Pailot, A., Reiling, N., Goldmann, O., Binz, T., Wegner, A., Tallam, A., Rausell, A. Immune-responsive gene 1 protein links metabolism to immunity by catalyzing itaconic acid production. Proceedings of the National Academy of Sciences 2013; 110: 7820-7825.

30. Rodríguez-Prados, J.-C., Través, P. G., Cuenca, J., Rico, D., Aragonés, J., Martín-Sanz, P., Cascante, M., Boscá, L. Substrate Fate in Activated Macrophages: A Comparison between Innate, Classic, and Alternative Activation. The Journal of Immunology 2010; 185: 605.

31. Palsson-McDermott, E. M., Curtis, A. M., Goel, G., Lauterbach, M. A. R., Sheedy, F. J., Gleeson, L. E., van den Bosch, M. W. M., Quinn, S. R., Domingo-Fernandez, R., Johnston, D. G. W., Jiang, J.-K., Israelsen, W. J., Keane, J., Thomas, C., Clish, C., Vander Heiden, M., Xavier, R. J., O'Neill, L. A. J. Pyruvate kinase $\mathrm{M} 2$ regulates Hif- $1 \alpha$ activity and IL-1 $\beta$ induction and is a critical determinant of the warburg effect in LPS-activated macrophages. Cell metabolism 2015; 21: 65-80.

32. Huang, S. C.-C., Everts, B., Ivanova, Y., O'Sullivan, D., Nascimento, M., Smith, A. M., Beatty, W., Love-Gregory, L., Lam, W. Y., O'Neill, C. M., Yan, C., Du, H., Abumrad, N. A., Urban, J. F., Jr., Artyomov, M. N., Pearce, E. L., Pearce, E. J. Cell-intrinsic lysosomal lipolysis is essential for alternative activation of macrophages. Nature immunology 2014; 15: 846-855.

33. Tan, Z., Xie, N., Cui, H., Moellering, D. R., Abraham, E., Thannickal, V. J., Liu, G. Pyruvate dehydrogenase kinase 1 participates in macrophage polarization via regulating glucose metabolism. The Journal of immunology 2015; 194: 6082-6089.

34. Malandrino, M. I., Fucho, R., Weber, M., Calderon-Dominguez, M., Mir, J. F., Valcarcel, L., Escoté, X., Gómez-Serrano, M., Peral, B., Salvadó, L. Enhanced fatty acid oxidation in adipocytes and macrophages reduces lipid-induced triglyceride accumulation and inflammation. American Journal of Physiology-Endocrinology and Metabolism 2015; 308: E756-E769.

35. Rath, M., Müller, I., Kropf, P., Closs, E. I., Munder, M. Metabolism via arginase or nitric oxide synthase: two competing arginine pathways in macrophages. Frontiers in immunology 2014; 5: 532.

36. Chandra, P., He, L., Zimmerman, M., Yang, G., Köster, S., Ouimet, M., Wang, H., Moore, K. J., Dartois, V., Schilling, J. D., Philips, J. A. Inhibition of Fatty Acid Oxidation Promotes Macrophage Control of Mycobacterium tuberculosis. mBio 2020; 11: e01139-20. 
37. Van den Bossche, J., Baardman, J., Otto, N. A., van der Velden, S., Neele, A. E., van den Berg, S. M., Luque-Martin, R., Chen, H.-J., Boshuizen, M. C., Ahmed, M. Mitochondrial dysfunction prevents repolarization of inflammatory macrophages. Cell reports 2016; 17: 684-696.

38. Anders, C. B., Lawton, T. M. W., Ammons, M. C. B. Metabolic immunomodulation of macrophage functional plasticity in nonhealing wounds. Current opinion in infectious diseases 2019; 32: 204-209.

39. Tomfohr, J., Lu, J., Kepler, T. B. Pathway level analysis of gene expression using singular value decomposition. BMC bioinformatics 2005; 6: 225-225.

40. Mudunuri, U., Che,A., Yi,M. and Stephens,R.M bioDBnet: the biological database network. Bioinformatics 2009; 25: 2.

41. Huang, D. W., Sherman, B. T., Lempicki, R. A. Systematic and integrative analysis of large gene lists using DAVID bioinformatics resources. Nature Protocols 2009; 4: 44-57.

42. Chong, J., Wishart, D. S., Xia, J. Using MetaboAnalyst 4.0 for Comprehensive and Integrative Metabolomics Data Analysis. Current Protocols in Bioinformatics 2019; 68: e86.

43. Wiklund, S., Johansson, E., Sjöström, L., Mellerowicz, E. J., Edlund, U., Shockcor, J. P., Gottfries, J., Moritz, T., Trygg, J. Visualization of GC/TOF-MS-Based Metabolomics Data for Identification of Biochemically Interesting Compounds Using OPLS Class Models. Analytical Chemistry 2008; 80: 115122.

44. Ambarus, C. A., Krausz, S., van Eijk, M., Hamann, J., Radstake, T. R. D. J., Reedquist, K. A., Tak, P. P., Baeten, D. L. P. Systematic validation of specific phenotypic markers for in vitro polarized human macrophages. Journal of Immunological Methods 2012; 375: 196-206.

45. Gause, W. C., Wynn, T. A., Allen, J. E. Type 2 immunity and wound healing: evolutionary refinement of adaptive immunity by helminths. Nature Reviews Immunology 2013; 13: 607.

46. Fan, J.-B., Miyauchi-Ishida, S., Arimoto, K.-i., Liu, D., Yan, M., Liu, C.-W., Győrffy, B., Zhang, D.-E. Type I IFN induces protein ISGylation to enhance cytokine expression and augments colonic inflammation. Proceedings of the National Academy of Sciences 2015; 112: 14313-14318.

47. Sudan, B., Wacker, M. A., Wilson, M. E., Graff, J. W. A systematic approach to identify markers of distinctly activated human macrophages. Frontiers in Immunology 2015; 6: 18.

48. Munn, D. H., Shafizadeh, E., Attwood, J. T., Bondarev, I., Pashine, A., Mellor, A. L. Inhibition of T cell proliferation by macrophage tryptophan catabolism. The Journal of experimental medicine 1999; 189: 1363-1372.

49. Tamada, M., Nagano, O., Tateyama, S., Ohmura, M., Yae, T., Ishimoto, T., Sugihara, E., Onishi, N., Yamamoto, T., Yanagawa, H., Suematsu, M., Saya, H. Modulation of Glucose Metabolism by CD44 Contributes to Antioxidant Status and Drug Resistance in Cancer Cells. Cancer research 2012; 72: 1438.

50. Nishiyama, A., Yokote, Y., Sakagami, H. Changes in Amino Acid Metabolism During Activation of Mouse Macrophage-like Cell Lines. In Vivo 2010; 24: 857-860.

51. Curran, J. N., Winter, D. C., Bouchier-Hayes, D. Biological fate and clinical implications of arginine metabolism in tissue healing. Wound Repair and Regeneration 2006; 14: 376-386.

52. Ji, L., Zhao, X., Zhang, B., Kang, L., Song, W., Zhao, B., Xie, W., Chen, L., Hu, X. Slc6a8-Mediated Creatine Uptake and Accumulation Reprogram Macrophage Polarization via Regulating Cytokine Responses. Immunity 2019; 51: 272-284.e7.

53. Kwon, H., Kim, S., Kim, Y., Lee, Y. The contribution of arachidonate 15-lipoxygenase in tissue macrophages to adipose tissue remodeling. Cell death \& disease 2016; 7: e2285-e2285.

54. Lefèvre, L., Galès, A., Olagnier, D., Bernad, J., Perez, L., Burcelin, R., Valentin, A., Auwerx, J., Pipy, B., Coste, A. PPAR $\gamma$ ligands switched high fat diet-induced macrophage M2b polarization toward M2a thereby improving intestinal Candida elimination. PloS one 2010; 5: e12828-e12828.

55. Talamonti, E., Pauter, A. M., Asadi, A., Fischer, A. W., Chiurchiù, V., Jacobsson, A. Impairment of systemic DHA synthesis affects macrophage plasticity and polarization: implications for DHA supplementation during inflammation. Cellular and Molecular Life Sciences 2017; 74: 2815-2826. 
56. Girodet, P.-O., Nguyen, D., Mancini, J. D., Hundal, M., Zhou, X., Israel, E., Cernadas, M. Alternative macrophage activation is increased in asthma. American journal of respiratory cell and molecular biology 2016; 55: 467-475.

57. Xie, M., Yu, Y., Kang, R., Zhu, S., Yang, L., Zeng, L., Sun, X., Yang, M., Billiar, T. R., Wang, H., Cao, L., Jiang, J., Tang, D. PKM2-dependent glycolysis promotes NLRP3 and AIM2 inflammasome activation. Nature Communications 2016; 7: 13280.

58. Millet, P., Vachharajani, V., McPhail, L., Yoza, B., McCall, C. E. GAPDH binding to TNF- $\alpha$ mRNA contributes to posttranscriptional repression in monocytes: a novel mechanism of communication between inflammation and metabolism. The Journal of Immunology 2016; 196: 2541-2551.

59. Bae, S., Kim, H., Lee, N., Won, C., Kim, H.-R., Hwang, Y.-i., Song, Y. W., Kang, J. S., Lee, W. J. $\alpha-$ Enolase expressed on the surfaces of monocytes and macrophages induces robust synovial inflammation in rheumatoid arthritis. The Journal of Immunology 2012; 189: 365-372.

60. Cordes, T., Wallace, M., Michelucci, A., Divakaruni, A. S., Sapcariu, S. C., Sousa, C., Koseki, H., Cabrales, P., Murphy, A. N., Hiller, K. Immunoresponsive gene 1 and itaconate inhibit succinate dehydrogenase to modulate intracellular succinate levels. Journal of Biological Chemistry 2016; 291: 14274-14284.

61. Lampropoulou, V., Sergushichev, A., Bambouskova, M., Nair, S., Vincent, E. E., Loginicheva, E., Cervantes-Barragan, L., Ma, X., Huang, S. C.-C., Griss, T. Itaconate links inhibition of succinate dehydrogenase with macrophage metabolic remodeling and regulation of inflammation. Cell metabolism 2016; 24: 158-166.

62. Littlewood-Evans, A., Sarret, S., Apfel, V., Loesle, P., Dawson, J., Zhang, J., Muller, A., Tigani, B., Kneuer, R., Patel, S. GPR91 senses extracellular succinate released from inflammatory macrophages and exacerbates rheumatoid arthritis. Journal of Experimental Medicine 2016; 213: 1655-1662.

63. Bozza, P. T., Magalhães, K. G., Weller, P. F. Leukocyte lipid bodies - Biogenesis and functions in inflammation. Biochimica et biophysica acta 2009; 1791: 540-551.

64. Infantino, V., Iacobazzi, V., Menga, A., Avantaggiati, M. L., Palmieri, F. A key role of the mitochondrial citrate carrier (SLC25A1) in TNF $\alpha$-and IFN $\gamma$-triggered inflammation. Biochimica et Biophysica Acta (BBA)-Gene Regulatory Mechanisms 2014; 1839: 1217-1225.

65. Lee, J.-H., Phelan, P., Shin, M., Oh, B.-C., Han, X., Im, S.-S., Osborne, T. F. SREBP-1a-stimulated lipid synthesis is required for macrophage phagocytosis downstream of TLR4-directed mTORC1. Proceedings of the National Academy of Sciences 2018; 115: E12228-E12234.

66. Driscoll, W. S., Vaisar, T., Tang, J., Wilson, C. L., Raines, E. W. Macrophage ADAM17 deficiency augments CD36-dependent apoptotic cell uptake and the linked anti-inflammatory phenotype. Circulation research 2013; 113: 52-61.

67. Sallusto, F., Cella, M., Danieli, C., Lanzavecchia, A. Dendritic cells use macropinocytosis and the mannose receptor to concentrate macromolecules in the major histocompatibility complex class II compartment: downregulation by cytokines and bacterial products. The Journal of experimental medicine 1995; 182: 389-400.

68. Lee, S. J., Evers, S., Roeder, D., Parlow, A. F., Risteli, J., Risteli, L., Lee, Y. C., Feizi, T., Langen, H., Nussenzweig, M. C. Mannose Receptor-Mediated Regulation of Serum Glycoprotein Homeostasis. Science 2002; 295: 1898.

69. Wilkinson, H. N., Roberts, E. R., Stafford, A. R., Banyard, K. L., Matteucci, P., Mace, K. A., Hardman, M. J. Tissue Iron Promotes Wound Repair via M2 Macrophage Polarization and the Chemokine (C-C Motif) Ligands 17 and 22. The American journal of pathology 2019; 189: 2196-2208.

70. Sironi, M., Martinez, F. O., D’Ambrosio, D., Gattorno, M., Polentarutti, N., Locati, M., Gregorio, A., Iellem, A., Cassatella, M. A., Van Damme, J., Sozzani, S., Martini, A., Sinigaglia, F., Vecchi, A., Mantovani, A. Differential regulation of chemokine production by Fc $\gamma$ receptor engagement in human monocytes: association of CCL1 with a distinct form of M2 monocyte activation (M2b, Type 2). Journal of Leukocyte Biology 2006; 80: 342-349. 
71. Tsuchimoto, Y., Asai, A., Tsuda, Y., Ito, I., Nishiguchi, T., Garcia, M. C., Suzuki, S., Kobayashi, M., Higuchi, K., Suzuki, F. M2b Monocytes Provoke Bacterial Pneumonia and Gut Bacteria-Associated Sepsis in Alcoholics. The Journal of Immunology 2015; 195: 5169-5177.

72. Yue, Y., Yang, X., Feng, K., Wang, L., Hou, J., Mei, B., Qin, H., Liang, M., Chen, G., Wu, Z. M2b macrophages reduce early reperfusion injury after myocardial ischemia in mice: A predominant role of inhibiting apoptosis via A20. International Journal of Cardiology 2017; 245: 228-235.

73. Yang, R., Liao, Y., Wang, L., He, P., Hu, Y., Yuan, D., Wu, Z., Sun, X. Exosomes Derived From M2b Macrophages Attenuate DSS-Induced Colitis. Frontiers in immunology 2019; 10.

74. Philippidis, P., Mason, J., Evans, B., Nadra, I., Taylor, K., Haskard, D., Landis, R. Hemoglobin scavenger receptor CD163 mediates interleukin-10 release and heme oxygenase-1 synthesis: antiinflammatory monocyte-macrophage responses in vitro, in resolving skin blisters in vivo, and after cardiopulmonary bypass surgery. Circulation research 2004; 94: 119-126.

75. Jenner, W., Motwani, M., Veighey, K., Newson, J., Audzevich, T., Nicolaou, A., Murphy, S., MacAllister, R., Gilroy, D. W. Characterisation of Leukocytes in a Human Skin Blister Model of Acute Inflammation and Resolution. PloS one 2014; 9: e89375.

76. Karna, E., Szoka, L., Huynh, T. Y. L., Palka, J. A. Proline-dependent regulation of collagen metabolism. Cellular and Molecular Life Sciences 2020; 77: 1911-1918.

77. Litwiniuk, M., Krejner, A., Speyrer, M. S., Gauto, A. R., Grzela, T. Hyaluronic acid in inflammation and tissue regeneration. Wounds 2016; 28: 78-88.

78. Galdiero, M. R., Garlanda, C., Jaillon, S., Marone, G., Mantovani, A. Tumor associated macrophages and neutrophils in tumor progression. Journal of cellular physiology 2013; 228: 1404-1412.

79. Wenes, M., Shang, M., Di Matteo, M., Goveia, J., Martín-Pérez, R., Serneels, J., Prenen, H., Ghesquière, B., Carmeliet, P., Mazzone, M. Macrophage Metabolism Controls Tumor Blood Vessel Morphogenesis and Metastasis. Cell Metabolism 2016; 24: 701-715.

80. Yan, D., Kowal, J., Akkari, L., Schuhmacher, A. J., Huse, J. T., West, B. L., Joyce, J. A. Inhibition of colony stimulating factor-1 receptor abrogates microenvironment-mediated therapeutic resistance in gliomas. Oncogene 2017; 36: 6049-6058.

81. Arts, R. J., Plantinga, T. S., Tuit, S., Ulas, T., Heinhuis, B., Tesselaar, M., Sloot, Y., Adema, G. J., Joosten, L. A., Smit, J. W. Transcriptional and metabolic reprogramming induce an inflammatory phenotype in non-medullary thyroid carcinoma-induced macrophages. Oncoimmunology 2016; 5: e1229725.

82. Liu, D., Chang, C., Lu, N., Wang, X., Lu, Q., Ren, X., Ren, P., Zhao, D., Wang, L., Zhu, Y. Comprehensive proteomics analysis reveals metabolic reprogramming of tumor-associated macrophages stimulated by the tumor microenvironment. Journal of Proteome Research 2017; 16: 288-297.

83. Xu, Z. Z., Xu, S., Kuhlmann, A., Kaech, S. M. The role of CD36 in macrophage lipid metabolism and function in tumor microenvironment. The Journal of Immunology 2020; 204: 240.9.

84. Vats, D., Mukundan, L., Odegaard, J. I., Zhang, L., Smith, K. L., Morel, C. R., Greaves, D. R., Murray, P. J., Chawla, A. Oxidative metabolism and PGC-1 $\beta$ attenuate macrophage-mediated inflammation. Cell metabolism 2006; 4: 13-24.

\section{Figure Legends:}


Figure 1: Macrophage Functional Phenotyping by Common Cell-Surface Markers. Cell surface marker profile of six MФ phenotypes were detected by FACS. CD14+ monocytes were isolated from human bloodderived PBMCs, differentiated into resting МФ (M0, shown in blue) with M-CSF ex vivo, and polarized into five activated phenotypes using IFN- $\gamma /$ LPS (M1, shown in red), IL-4/IL-13 (M2a, shown in yellow), IC/LPS (M2b, shown in green), IL-10 (M2c, shown in gray), or IL-6/LIF (M2d, shown in purple) for 72 hours. FACS gating on four common $\mathrm{M} \Phi$ cell surface markers for all six $\mathrm{M} \Phi$ phenotypes is shown as live cell count for each marker gate (A-D) and mean fluorescence intensity (MFI) profile of gate (E-H). The cell surface markers depicted include CD40 (A, E), CD86 (B, F), CD163 (C, G), and HLA-DR MHC class II receptor (D, H). Histograms are total cell count and representative of three biological replicates. MFI is normalized to total cell count and includes all three biological replicates $(\mathrm{N}=3$, mean $\pm \mathrm{SEM})$.

\section{Figure 2: МФ Functional Phenotyping by Gene and Protein Expression of Immunomodulatory Factors.}

Multiplex detection of immunomodulatory factors in six MФ phenotypes harvested at 24 hours were detected using magnetic bead-based quantification of mRNA and secreted protein. CD14+ monocytes were isolated from human blood-derived PBMCs, differentiated into resting MФ (M0, shown in blue) with M-CSF ex vivo, and polarized into five activated phenotypes using IFN- $\gamma /$ LPS (M1, shown in red), IL-4/IL-13 (M2a, shown in yellow), IC/LPS (M2b, shown in green), IL-10 (M2c, shown in gray), or IL-6/LIF (M2d, shown in purple). Gene and protein expression profile (diamond-whisker plots and bar charts, respectively) of key functional molecules were profiled by multiplex assay after 24 hours of ex vivo polarization. Immunomodulatory function includes pro-inflammatory (TNF $\alpha$, IL-12p70, CCL3, CXCL10, CXCL8, and IFN $\alpha$ ) (A), immuneregulatory/tissue-repair (IL-10, IL-6, IL-1b, IL-1 $\alpha$, TGF-b, and CCL2) (B), and growth factors (GM-CSF, PDGF-BB, and VEGF-A) (C). Diamond-whisker plots display 25\%-75\% quartile range, median, and mean. Bar charts indicate mean \pm SEM. Expression profiles were normalized to total cell count and include three biological replicates $(\mathrm{N}=3)$. 


\section{Figure 3: МФ Polarization into Functional Phenotypes Activates Distinct Gene Expression Profiles}

Relative to the Parent, Resting МФ Phenotype (M0). Gene set enrichment analysis (GSEA) and pathway impact scoring of global myeloid gene expression demonstrates distinct patterns in five polarized MФ phenotypes. Briefly, multiplexed myeloid gene expression was directly detected through molecular barcode probes and normalized to the geometric mean of the housekeeping gene set. GSEA for each phenotype was calculated for pathway impact relative to the non-polarized, resting $\mathrm{M} \Phi(\mathrm{M} 0)$. Radar plots for each polarized MФ phenotype display pathway score based on impact regardless of whether genes were up- or down-regulated (Undirected Global Significance [UGS], solid color) and pathway score based on impact incorporating t-statistic comparison of gene regulation as relatively increased or decreased (Directed Global Significance [DGS], hatch marks). Radar plots include UGS and DGS scores for all five polarized MФ phenotypes: M1 MФs (IFN- $\gamma /$ LPS treated, shown in red) (A), M2a MФs (IL-4/IL-13 treated, shown in yellow) (B), M2b MФs (IC/LPS treated, shown in green) (C), M2c MФs (IL-10 treated, shown in gray) (D), and M2d MФs (IL-6/LIF treated, shown in

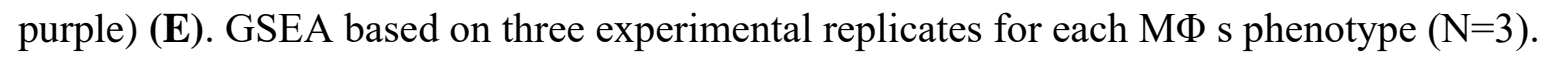

\section{Figure 4: Gene Expression Profile Comparison of Polarized MФ Phenotypes Identifies Unique and} Overlapping Immunomodulatory Functions. Gene Ontology (GO) annotation of global myeloid gene expression in five MФ phenotypes identifies relative biological functionality across polarized states.

Multiplexed myeloid gene expression profile of all five polarized $M \Phi$ phenotypes was used to annotate impacted biological functionality based on GO Processes. For each MФ phenotype, the top 15 impacted GO processes significantly enriched $(\mathrm{p} \leq 0.05)$ were plotted relative to the other four polarized $M \Phi$ phenotypes. Enrichment plots for prioritized GO process profile include all five polarized MФ phenotypes: M1 MФs (IFN$\gamma /$ LPS treated, shown in red) (A), M2a MФs (IL-4/IL-13 treated, shown in yellow) (B), M2b MФs (IC/LPS treated, shown in green) $(\mathbf{C}), \mathrm{M} 2 \mathrm{c}$ MФs (IL-10 treated, shown in gray) $(\mathbf{D})$, and M2d MФs (IL-6/LIF treated, shown in purple) (E). GO process annotation was based on three experimental replicates for each MФ phenotype $(\mathrm{N}=3)$. 


\section{Figure 5: Untargeted Metabolomics of Five Polarized MФ Phenotypes Identifies Biomarkers of Shared} and Unique Profile Relative to the Parent, Resting МФ Phenotype. Global metabolomics profile of the parent, resting $М \Phi$ phenotype (M0) and five polarized MФ phenotypes identified 498 compounds of known identity, normalized to cell count. MФ metabotype clustering was determined through Orthogonal Projections to Latent Structures Discriminant Analysis (OPLSDA), inset clustering scores plots with 2D T-scores (A.1, B.1, C.1, D.1, \& E.1). S-plot comparison of each polarized $M \Phi$ phenotype was plotted relative to the resting, parent MФ phenotype (denoted in blue) (A.1, B.1, C.1, D.1, \& E.1) and normalized peak intensity for biomarkers of particular note (a, b, c) are displayed as box-whisker plots (A.2, B.2, C.2, D.2, \& E.2). Multivariate, cluster analysis is displayed for all five polarized MФ phenotypes: M1 MФs (IFN- $\gamma /$ LPS treated, shown in red) (A), M2a MФs (IL-4/IL-13 treated, shown in yellow) (B), M2b MФs (IC/LPS treated, shown in green) (C), M2c MФs (IL-10 treated, shown in gray) (D), and M2d MФs (IL-6/LIF treated, shown in purple) (E). Untargeted metabolomics profiles are based on three experimental replicates for each $M \Phi$ phenotype $(\mathrm{N}=3)$.

\section{Figure 6: Global Metabolomics Comparison of the Classically Activated МФ Phenotype (M1 МФ) to} Four Subtypes of Alternately Activated MФs (M2a, M2b, M2c, \& M2d MФ). OPLSDA clustering relative to the parent, resting $\mathrm{M} \Phi$ phenotype $(\mathrm{M} 0 \mathrm{M} \Phi)$ identified metabolite biomarkers correlated with all five polarized MФ phenotypes based on OPLSDA variable influence on projection (VIP) values. VIP values were used to identified both shared and unique metabolite biomarkers of the classically-activated M1 M $\Phi$ phenotype and four subtypes of the alternately-activated M2 MФ phenotypes, displayed as OPLSDA Shared and Unique Structures (SUS) plots (A.1, B.1, C.1, and D.1). For each SUS plot, metabolite biomarkers uniquely correlated to the M1 MФ phenotype (red diamonds) are plotted along the x-axis and those metabolite biomarkers uniquely correlated to the M2 MФ phenotype are plotted along the y-axis. Shared metabolite biomarkers (black circles) are plotted along the diagonals, reflecting biomarker positive/negative correlation to each polarized MФ phenotype relative to the parent, resting $М \Phi$ phenotype (M0 M $\Phi)$. Associated biomarkers and correlation 
scores are shown in matching tables (A.2, B.2, C.2, and D.2). OPLSDA SUS plots are displayed for four polarized M2 MФ phenotypes, relative to the M1 MФs (IFN- $\gamma /$ LPS treated, shown in red): M2a MФs (IL-4/IL13 treated, shown in yellow) (A), M2b MФs (IC/LPS treated, shown in green) (B), M2c MФs (IL-10 treated, shown in gray) (C), and M2d MФs (IL-6/LIF treated, shown in purple) (D). Untargeted metabolomics profiles are based on three experimental replicates for each $\mathrm{M} \Phi$ phenotype $(\mathrm{N}=3)$.

\section{Figure 7: МФ Polarization into Functional Phenotypes Activates Distinct Metabolic Pathways.}

Untargeted, global metabolomics profile of polarized MФ phenotypes identified 498 known compounds, normalized to total cell count, and profiled relative to the resting M0 MФ phenotype. For normalized, M0relative metabolite profiles, over-representation analysis by hypergeometric testing and pathway topology analysis by two node centrality measures (degree centrality and betweenness centrality) was performed for each polarized MФ phenotype including M1 MФs (IFN- $\gamma /$ LPS treated, shown in red) (A), M2a MФs (IL-4/IL-13 treated, shown in yellow) (B), M2b MФs (IC/LPS treated, shown in green) (C), M2c MФs (IL-10 treated, shown in gray) (D), and M2d MФs (IL-6/LIF treated, shown in purple) (E). Metabolic Pathway Topology is plotted as Pathway Impact (x-axis) and significance of pathway topography (-Log [p value], y-axis) for each polarized MФ phenotype.

\section{Figure 8: МФ Polarization into Functional Phenotypes Activates Distinct Metabolic Gene Expression}

Profiles Relative to the Parent, Resting МФ Phenotype (M0). Gene set enrichment analysis (GSEA) and pathway impact scoring of global myeloid gene expression demonstrates distinct patterns in metabolism-related transcripts in the five polarized $\mathrm{M} \Phi$ phenotypes. Associated metabolism gene expression profiles for most impacted pathways are shown including central metabolism (A), amino acid metabolism (B), fatty acid metabolism (C), arachidonic acid metabolism (D), and miscellaneous pathways (E). Red bars represent gene targets that are differentially regulated $(\mathrm{p} \leq 0.05)$ when compared to the M0 phenotype. Myeloid gene 
expression was directly detected through molecular barcode probes and normalized to the geometric mean of the housekeeping gene set. Gene expression is mean \pm SEM. Pathway topology and metabolic gene expression profiles are based on triplicate, experimental replicates $(\mathrm{N}=3)$.

\section{Figure 9: Schematic Demonstrating Metabolic Pathway Flux Relative to the Parent, Resting MФ}

Phenotype (M0). Metabolic flux of selected metabolites within the specified pathways are denoted by arrow direction. Each of the colored boxes above the listed metabolite represents fold change relative to the parent M0 MФs $\left(1<\log _{2}<-1\right)$. Fold change values $\left(-\log _{2}<\mathrm{FC}>\log _{2}\right)$ are denoted in gray. Fold change values $\left(-\log _{2}<\right.$ FC) are denoted in shades of green and fold change values ( $\mathrm{FC}>\log _{2}$ ) are denoted in gray shades of blue.

\section{Figure 10: МФ Functional Phenotyping at 72 Hours Post Polarization by Gene and Protein Expression of}

Immunomodulatory Factors. Multiplex detection of immunomodulatory factors in six M $\Phi$ phenotypes harvested at 72 hours were detected using magnetic bead-based quantification of mRNA and secreted protein. Immunomodulatory function includes pro-inflammatory (TNF $\alpha$, IL-12p70, CCL3, CXCL10, CXCL8, and IFN $\alpha)(\mathbf{A})$, immune-regulatory/tissue-repair (IL-10, IL-6, IL-1b, IL-1 $\alpha$, TGF-b, and CCL2) (B), and growth factors (GM-CSF, PDGF-BB, and VEGF-A) (C) for the M0 resting MФs (shown in blue), M1 MФs (IFN- $\gamma / \mathrm{LPS}$ treated, shown in red), M2a MФs (IL-4/IL-13 treated, shown in yellow), M2b MФs (IC/LPS treated, shown in green), M2c MФs (IL-10 treated, shown in gray), and M2d MФs (IL-6/LIF treated, shown in purple). Diamondwhisker plots display 25\%-75\% quartile range, median, and mean. Bar charts indicate mean \pm SEM. Expression profiles were normalized to total cell count and include three biological replicates $(\mathrm{N}=3)$.

\section{Figure 11: Gene Expression Profile Comparison of Polarized MФ Phenotypes Identifies Unique and}

\section{Overlapping Immunomodulatory Functions between 24- and 72-hour Polarization Times. Gene Ontology}

(GO) annotation of global myeloid gene expression identifies the differential biological functionality between the 24 (light colored bars) and 72-hour (dark colored bars) time points in five MФ phenotypes. For each MФ phenotype, the top 15 impacted GO processes significantly enriched $(\mathrm{p} \leq 0.05)$ for both time points were plotted 
as such: M1 MФs (IFN- $\gamma /$ LPS treated, shown in red) (A), M2a MФs (IL-4/IL-13 treated, shown in yellow) (B), M2b MФs (IC/LPS treated, shown in green) (C), M2c MФs (IL-10 treated, shown in gray) (D), and M2d MФs (IL-6/LIF treated, shown in purple) (E). GO process annotation was based on three experimental replicates for each $\mathrm{M} \Phi$ s phenotype $(\mathrm{N}=3)$.

Figure 12: Metabotype Profiling of Polarized MФs at 24 and 72 Hours. Pathway impact scores derived from pathway topology analysis were compared at 24 and 72 hours for the M1 MФs (IFN- $\gamma /$ LPS treated, shown in red) (A), M2a MФs (IL-4/IL-13 treated, shown in yellow) (B), M2b MФs (IC/LPS treated, shown in green) (C), M2c MФs (IL-10 treated, shown in gray) (D), and M2d MФs (IL-6/LIF treated, shown in purple) (E). The top 15 enriched pathways are depicted in each spider plot with the hatched plots and solid plots representing the 24- and 72-hour time points, respectively. 


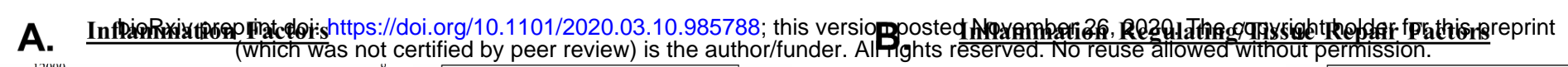
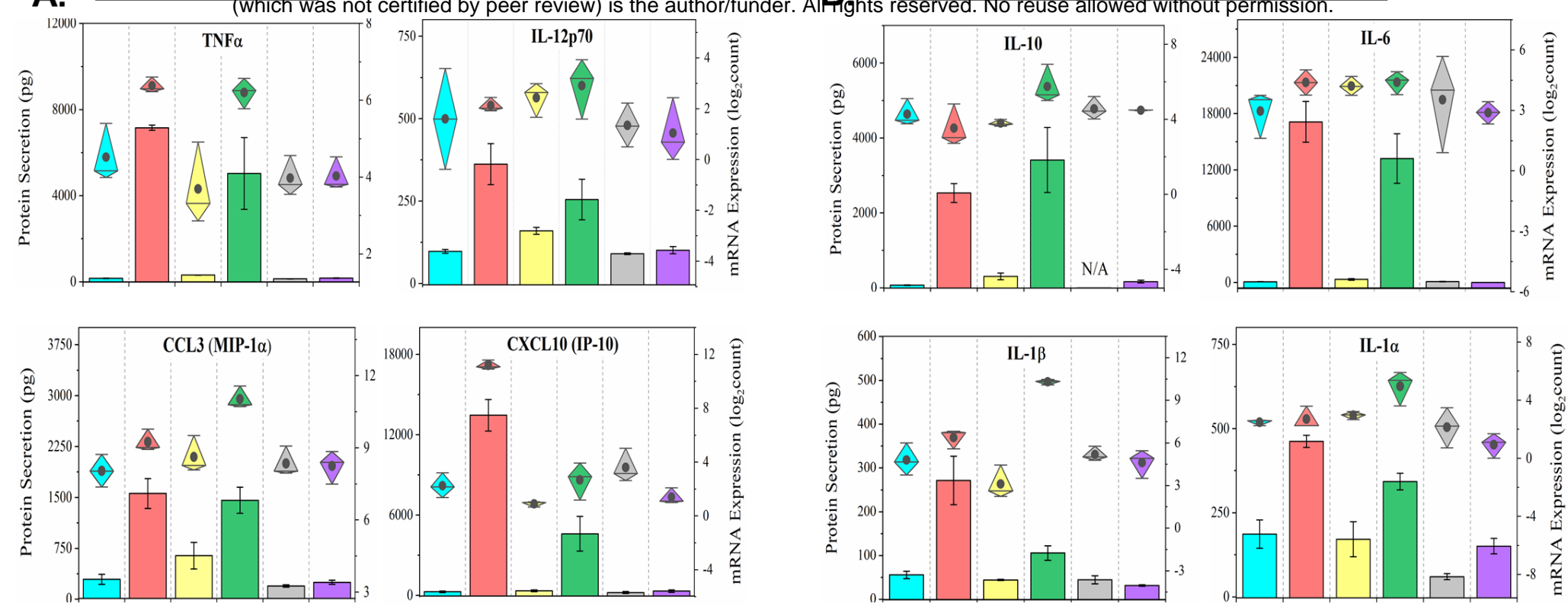

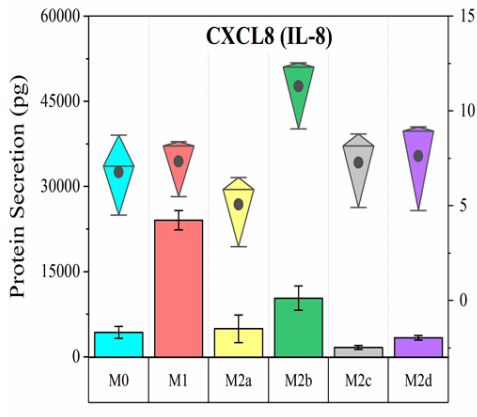

Phenotype

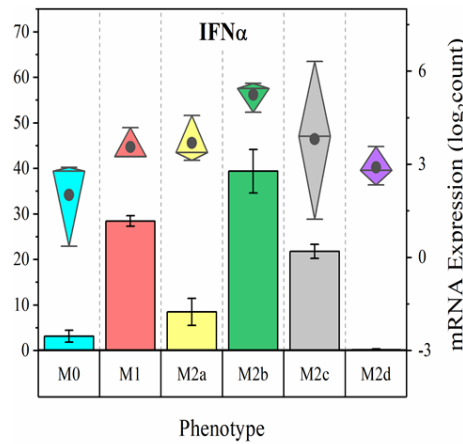

\section{Growth Factors}

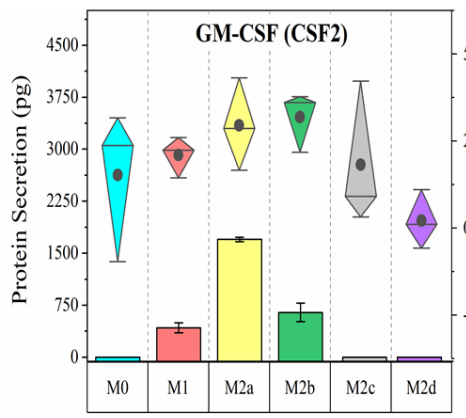

Phenotype

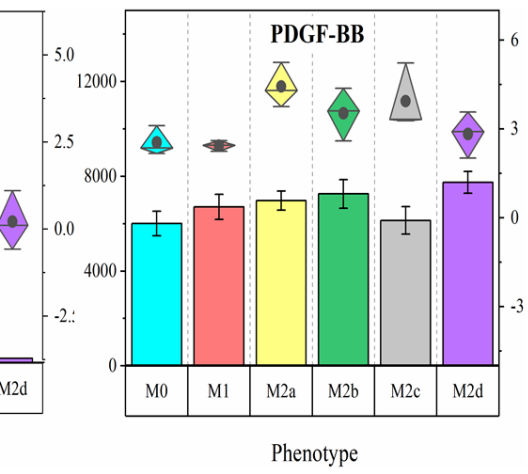

Legend

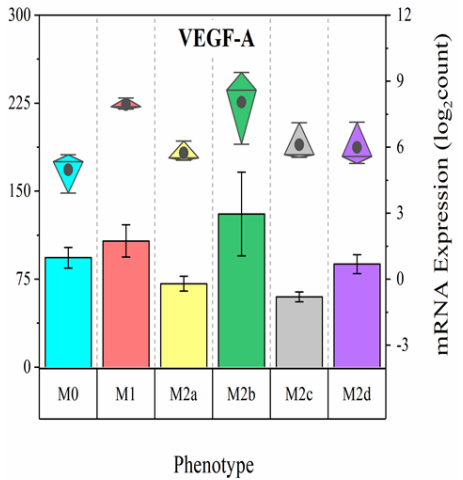

$\square \mathrm{M} 0 \bigotimes 25 \% \sim 75 \%$

I Range within 1.5IQR

- Median Line 

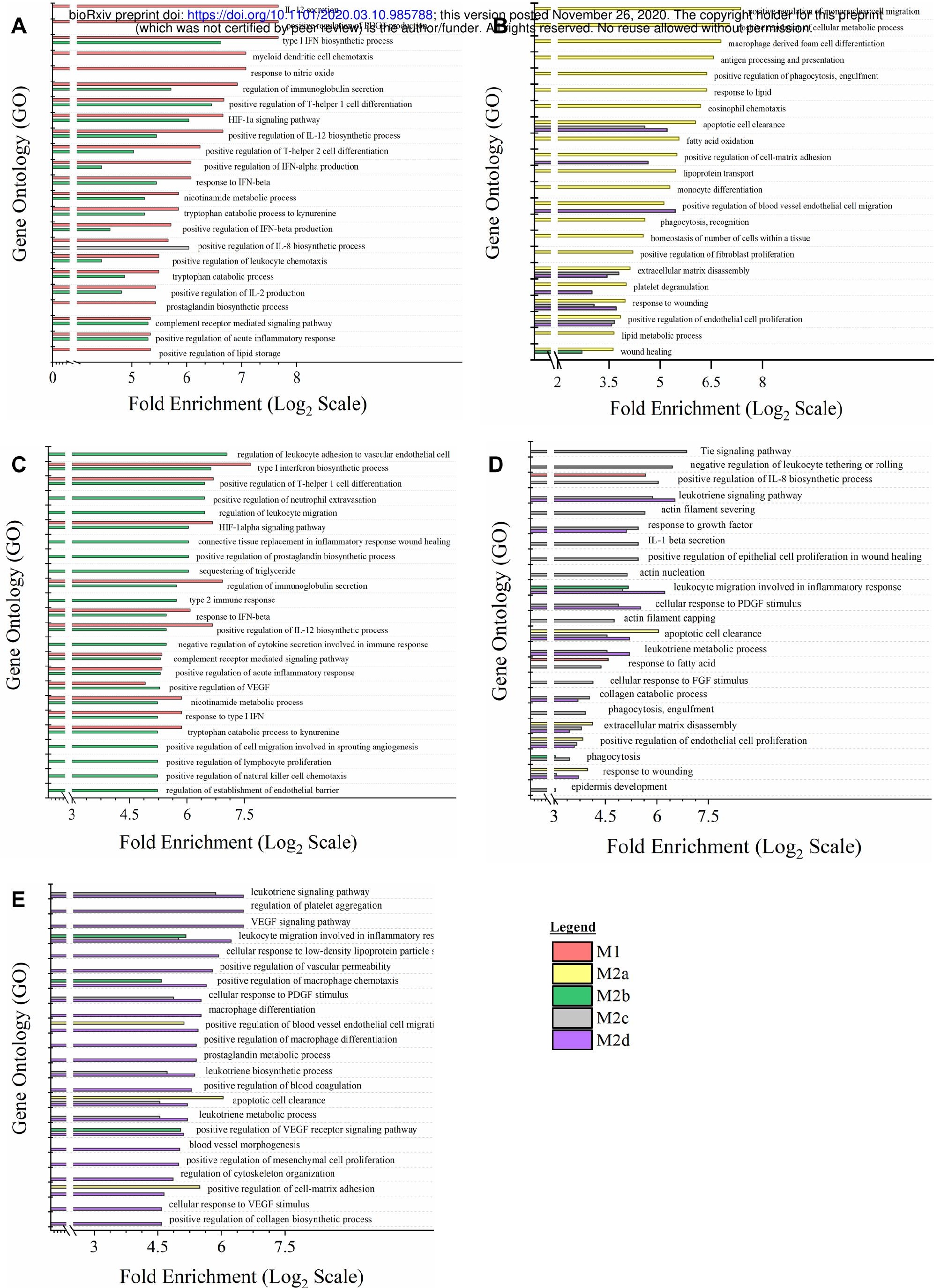
A. bioRxiy preprint doi: httpsi///doi.org/10.1101/2020.p3.10.985788;4hiइ version posted November 26, 2020. The copyright holder for this preprint

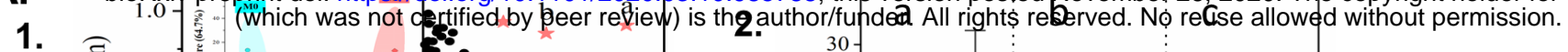
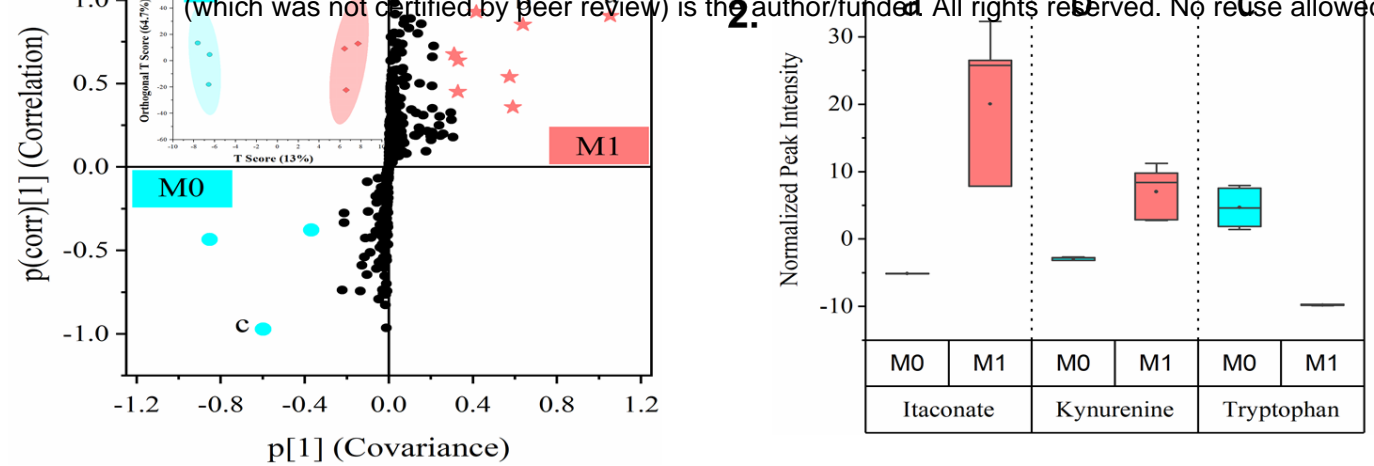

B.

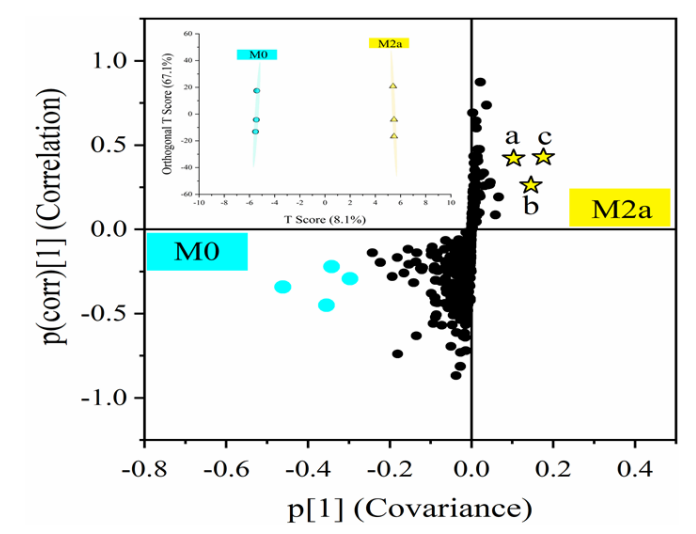

C

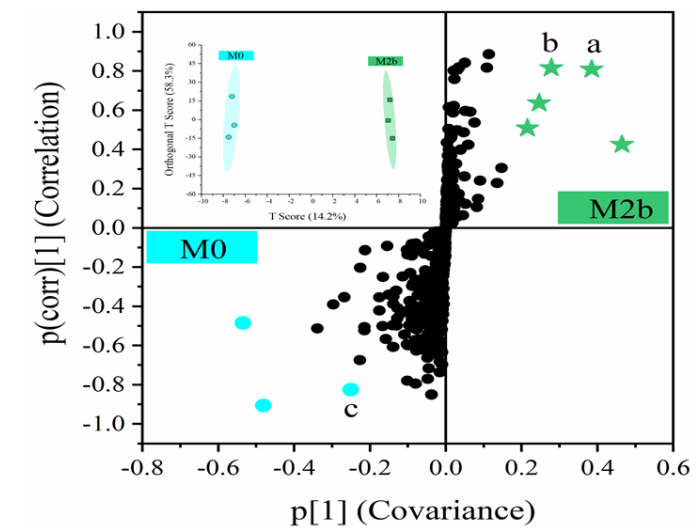

D

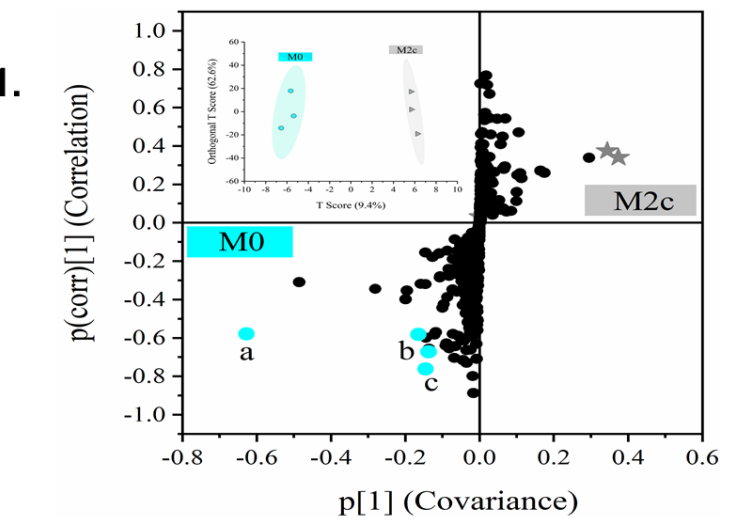

E

1.

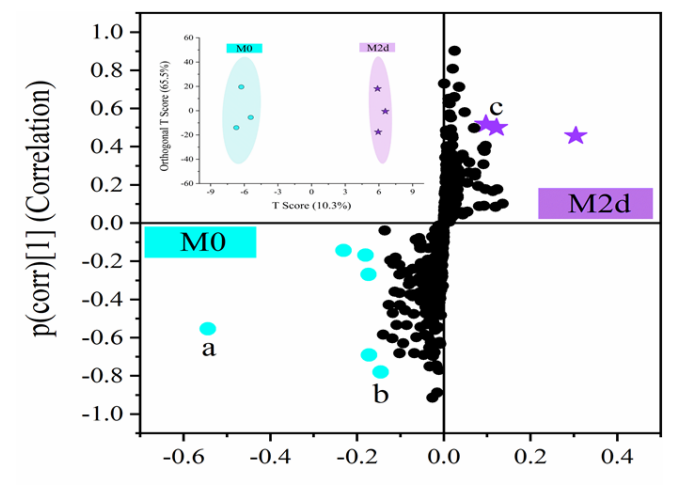

2.

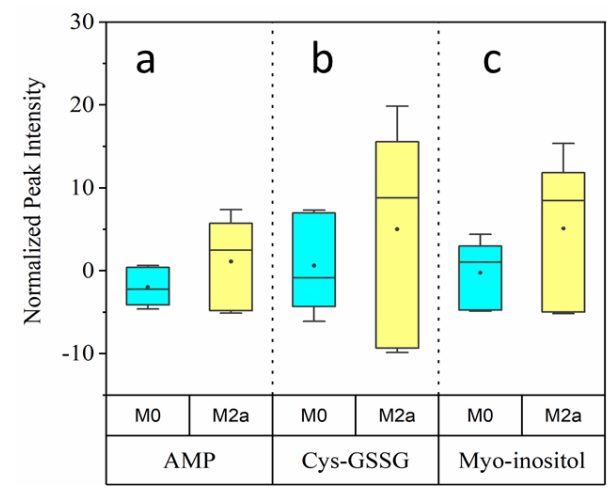

2.

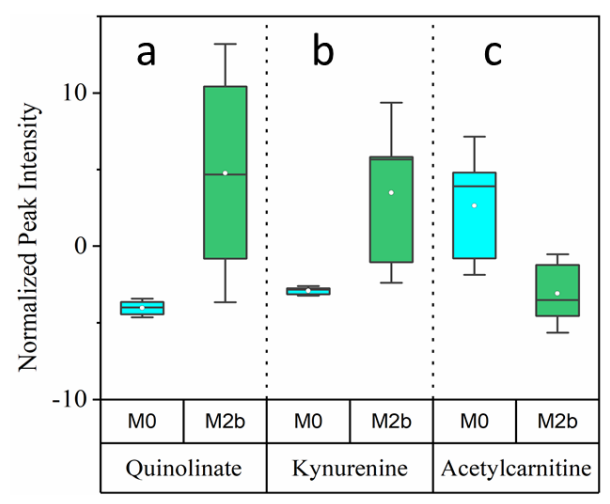

2.

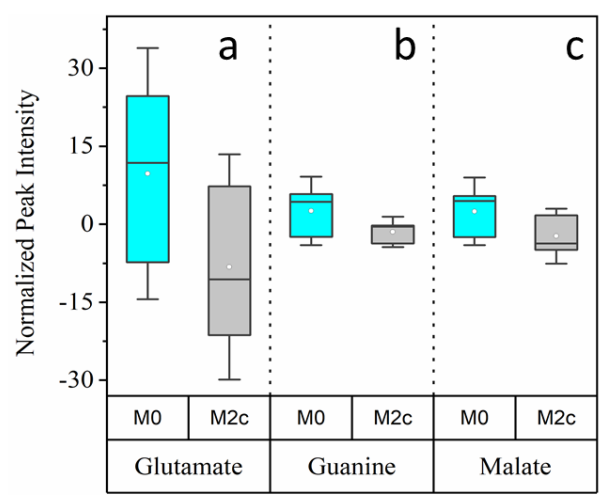

Legend

2.

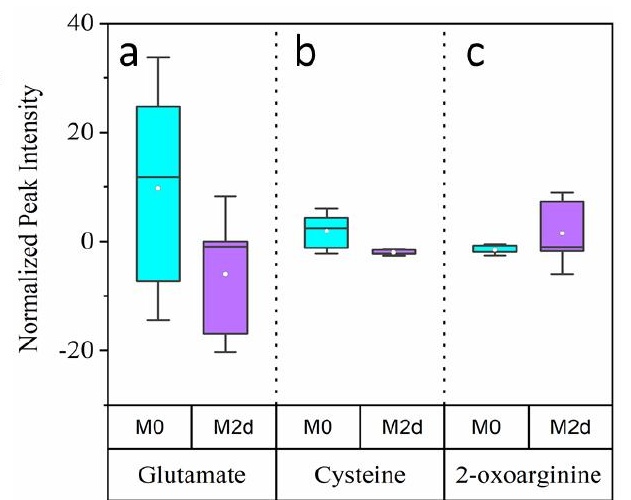

M1

M2a

$\mathrm{M} 2 \mathrm{~b}$

$\mathrm{M} 2 \mathrm{c}$

M2d

$25 \% \sim 75 \%$

I Mean $\pm 2 \mathrm{SE}$

- Median Line

- Mean 

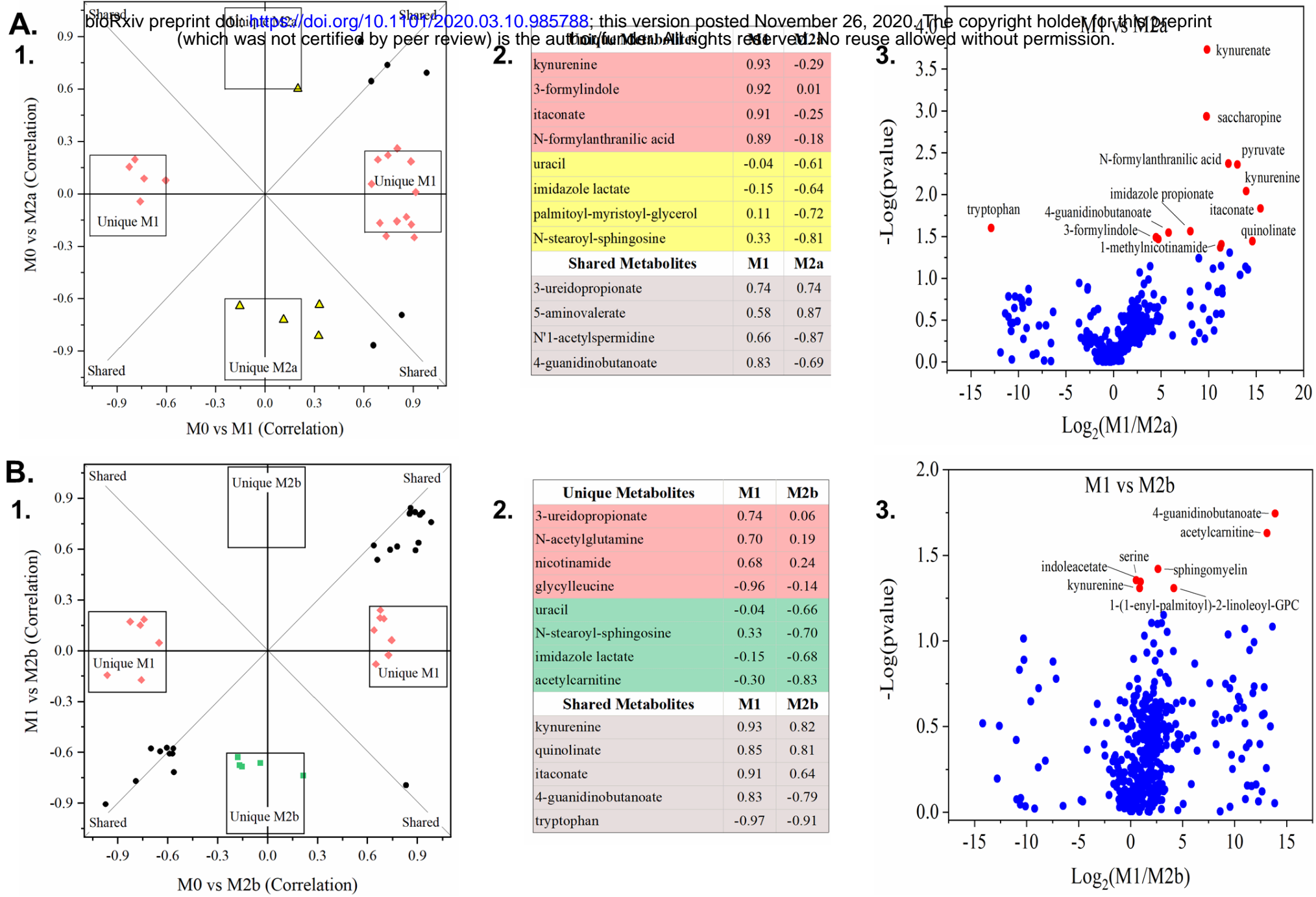

\begin{tabular}{|l|cc|}
\hline \multicolumn{1}{|c|}{ Unique Metabolites } & M1 & M2b \\
3-ureidopropionate & 0.74 & 0.06 \\
N-acetylglutamine & 0.70 & 0.19 \\
nicotinamide & 0.68 & 0.24 \\
glycylleucine & -0.96 & -0.14 \\
uracil & -0.04 & -0.66 \\
N-stearoyl-sphingosine & 0.33 & -0.70 \\
imidazole lactate & -0.15 & -0.68 \\
acetylearnitine & -0.30 & -0.83 \\
\hline \multicolumn{1}{|c|}{ Shared Metabolites } & M1 & M2b \\
\hline kynurenine & 0.93 & 0.82 \\
quinolinate & 0.85 & 0.81 \\
\hline itaconate & 0.91 & 0.64 \\
4-guanidinobutanoate & 0.83 & -0.79 \\
tryptophan & -0.97 & -0.91 \\
\hline
\end{tabular}
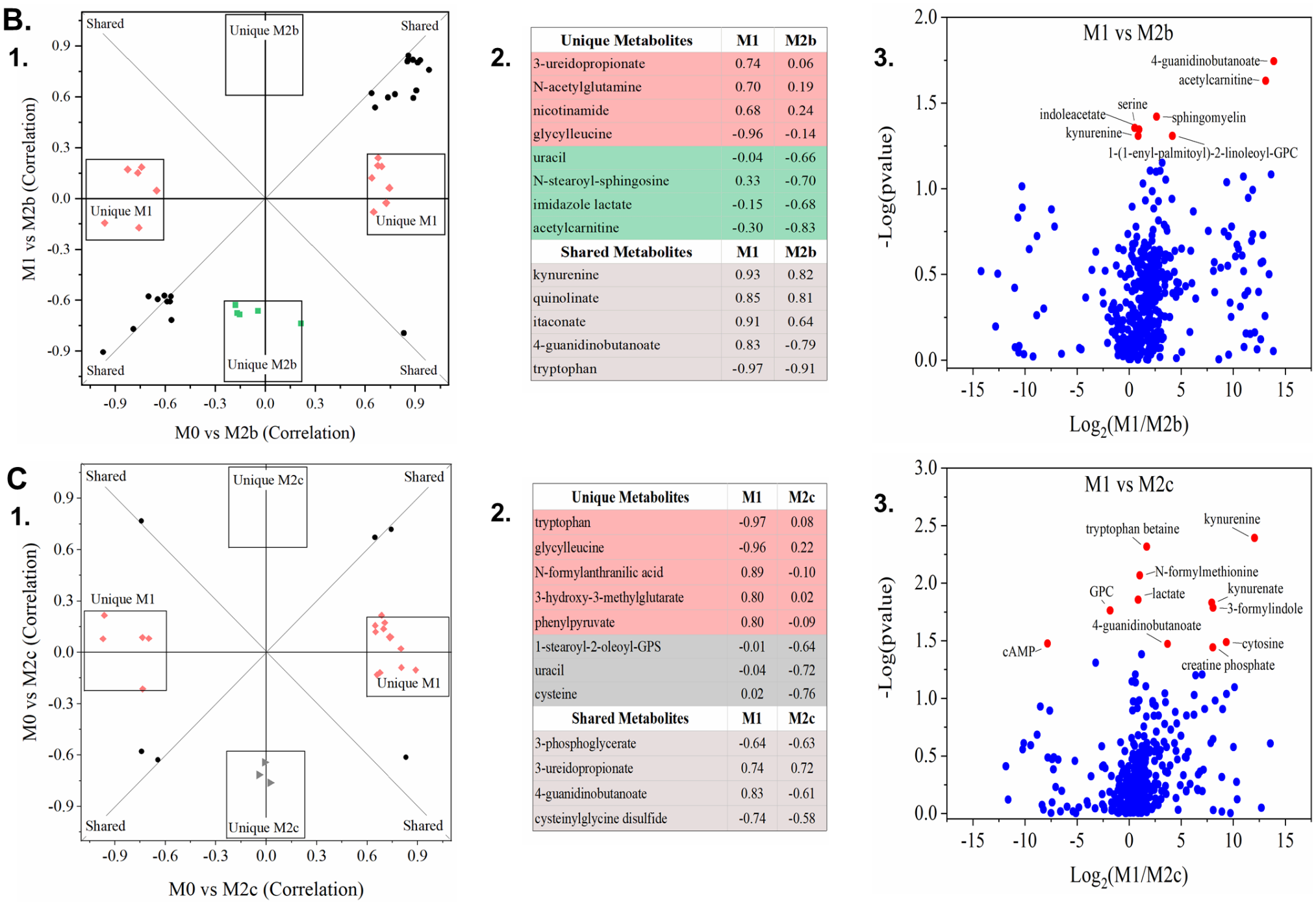

\begin{tabular}{|c|c|c|}
\hline 2. Unique Metabolites & M1 & M2c \\
tryptophni & -0.97 & 0.08
\end{tabular}
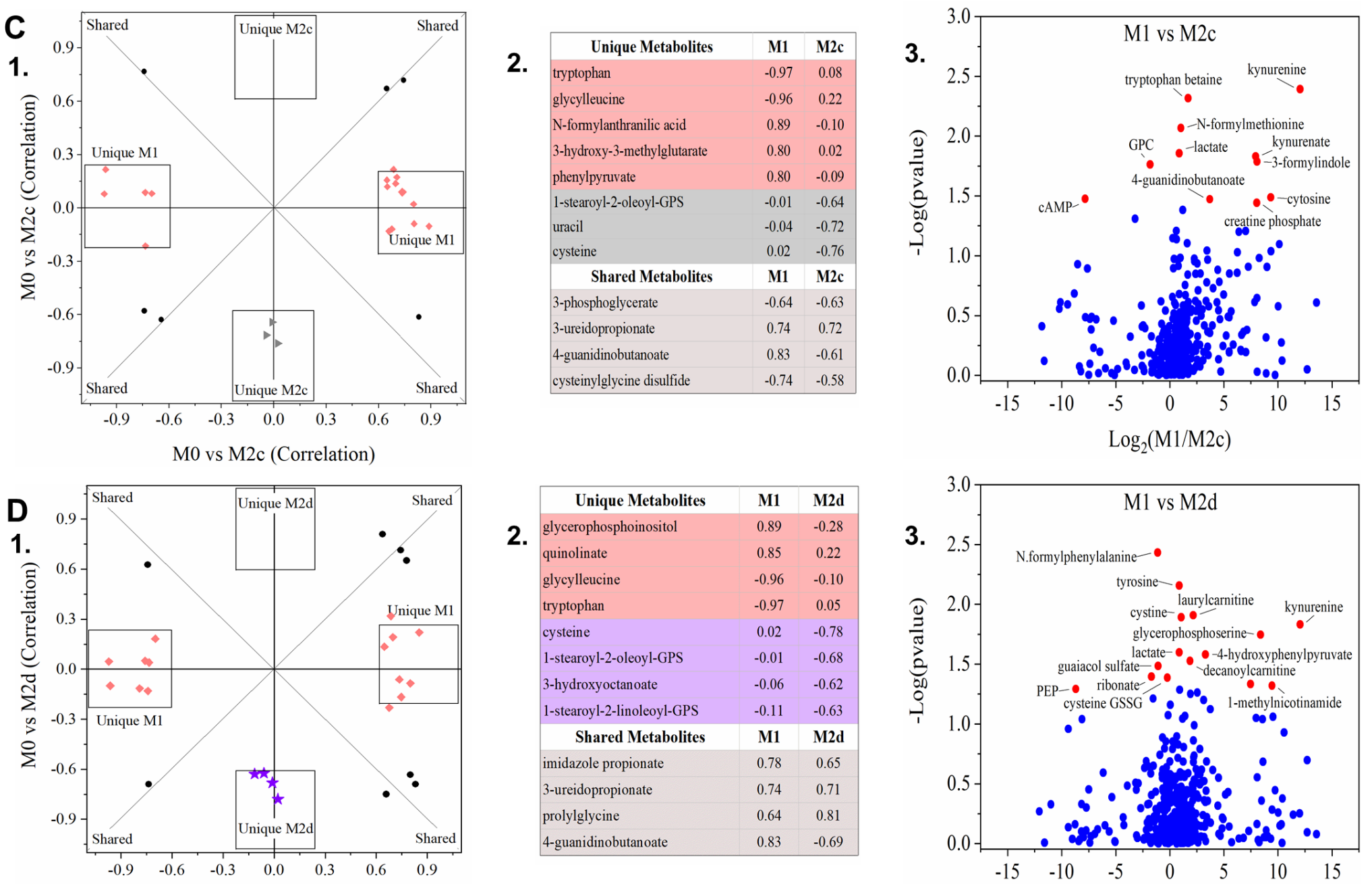

\begin{tabular}{|l|c|c|}
\hline \multicolumn{1}{|c|}{ Unique Metabolites } & M1 & M2d \\
glycerophosphoinositol & 0.89 & -0.28 \\
quinolinate & 0.85 & 0.22 \\
glycylleucine & -0.96 & -0.10 \\
tryptophan & -0.97 & 0.05 \\
cysteine & 0.02 & -0.78 \\
1-stearoyl-2-oleoyl-GPS & -0.01 & -0.68 \\
3-hydroxyoctanoate & -0.06 & -0.62 \\
1-stearoyl-2-linoleoyl-GPS & -0.11 & -0.63 \\
\hline \multicolumn{1}{|c|}{ Shared Metabolites } & M1 & M2d \\
\hline imidazole propionate & 0.78 & 0.65 \\
\hline 3-ureidopropionate & 0.74 & 0.71 \\
\hline prolylglycine & 0.64 & 0.81 \\
4-guanidinobutanoate & 0.83 & -0.69 \\
\hline
\end{tabular}

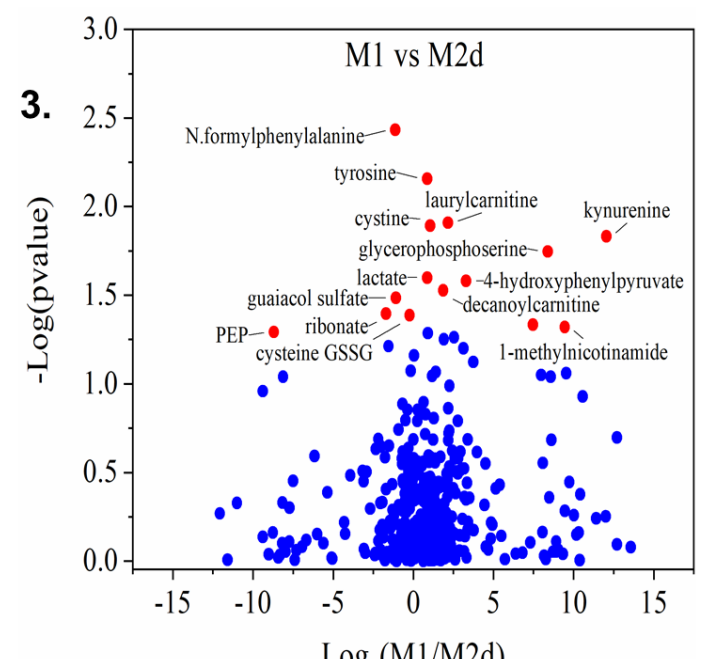




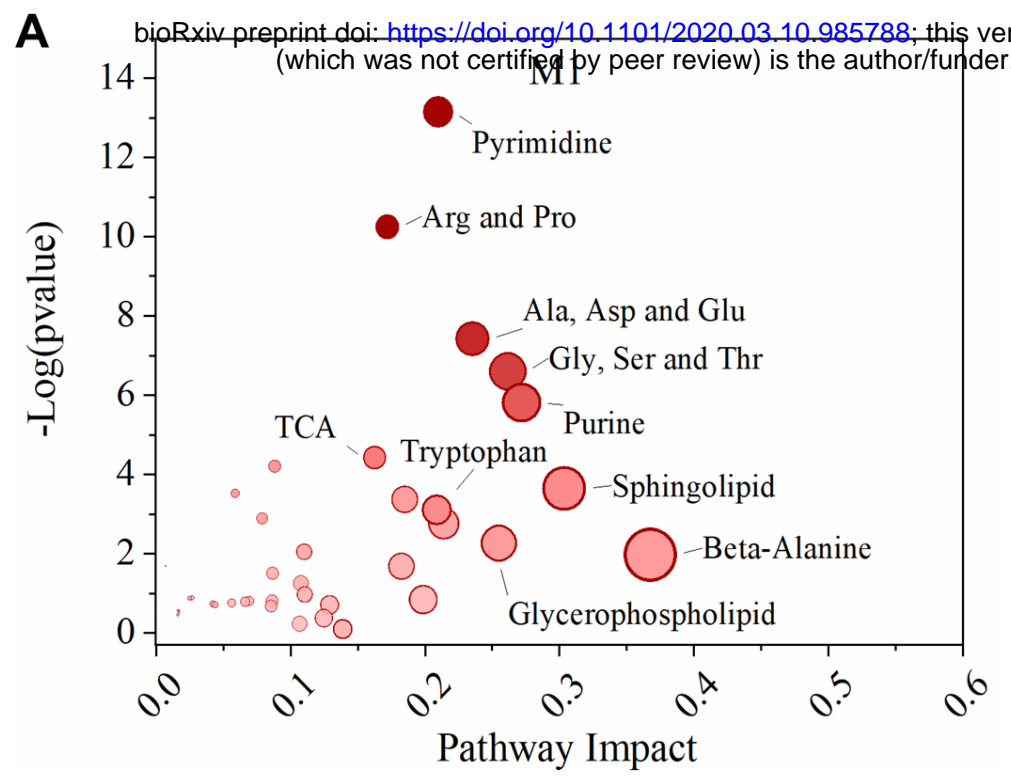

Bosted Nevember 26, 2020. The copyright holder for this preprint
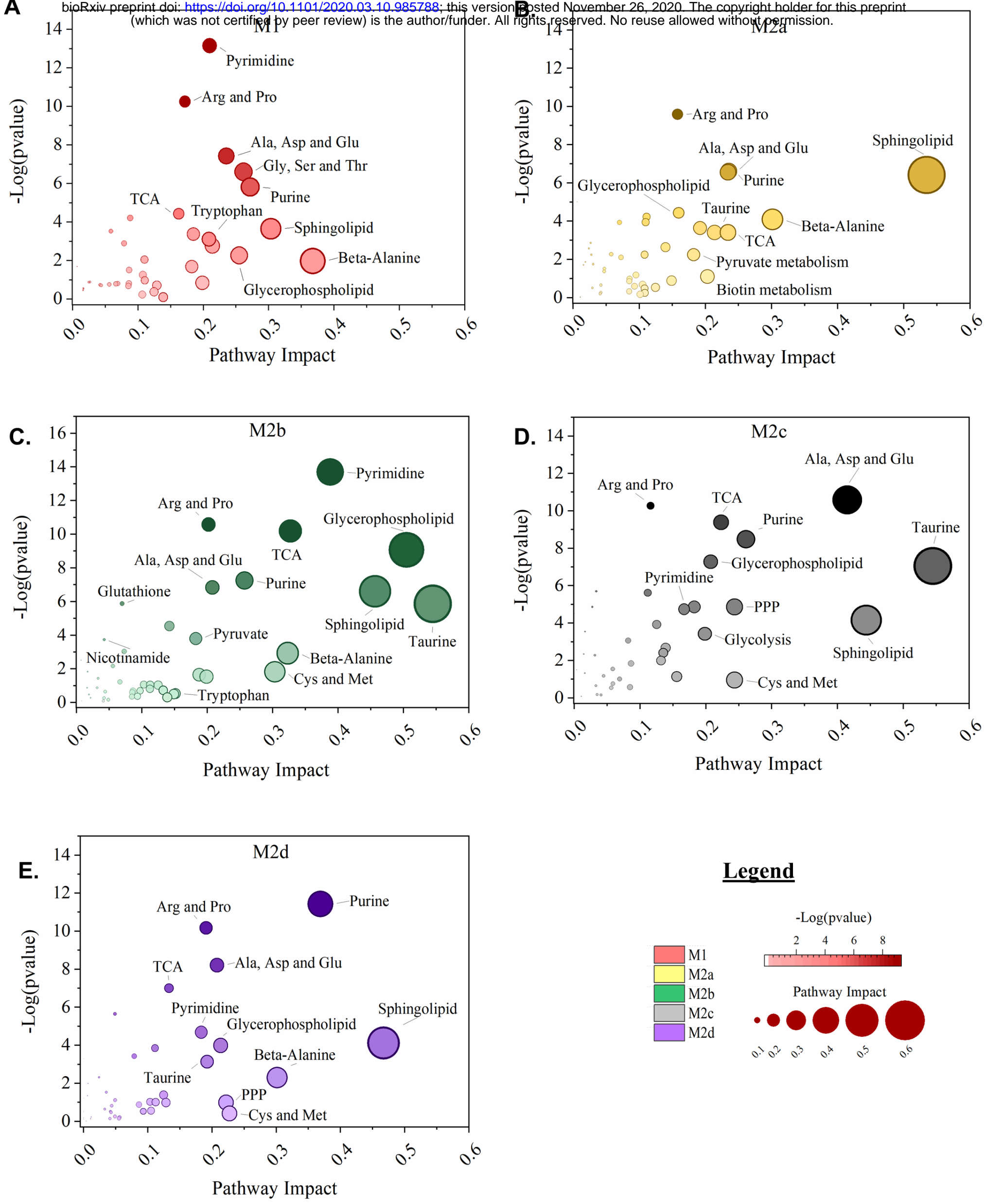

Legend

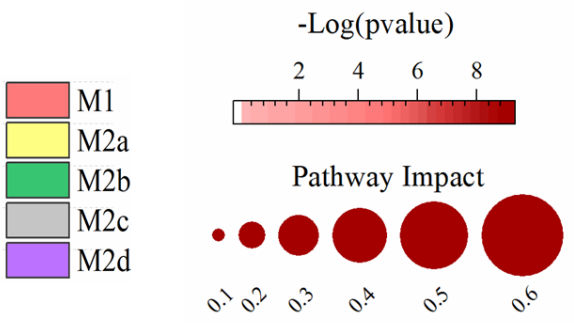


bioRxiv prepript doi: https://doi.prg/10.1101/2020 03 10.985788; this version posted Nevember 26, 2020. The copyright holder for this preprint

A ACLY (which was not certified by peer review) is the author/funder. All rights reserved. No reuse allowed without permission.

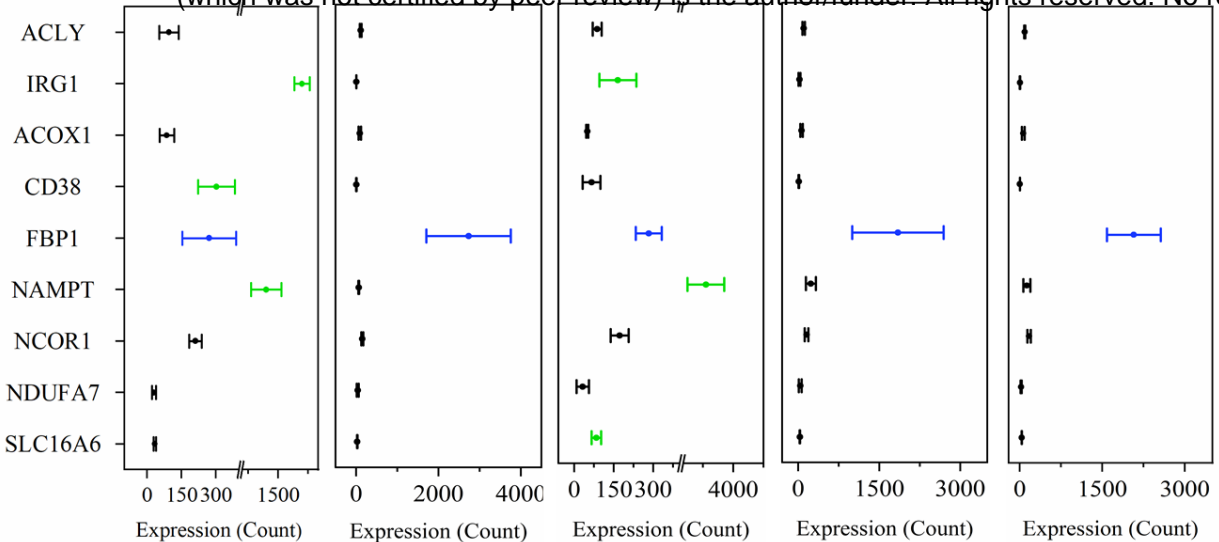

\section{Central \\ Metabolism}
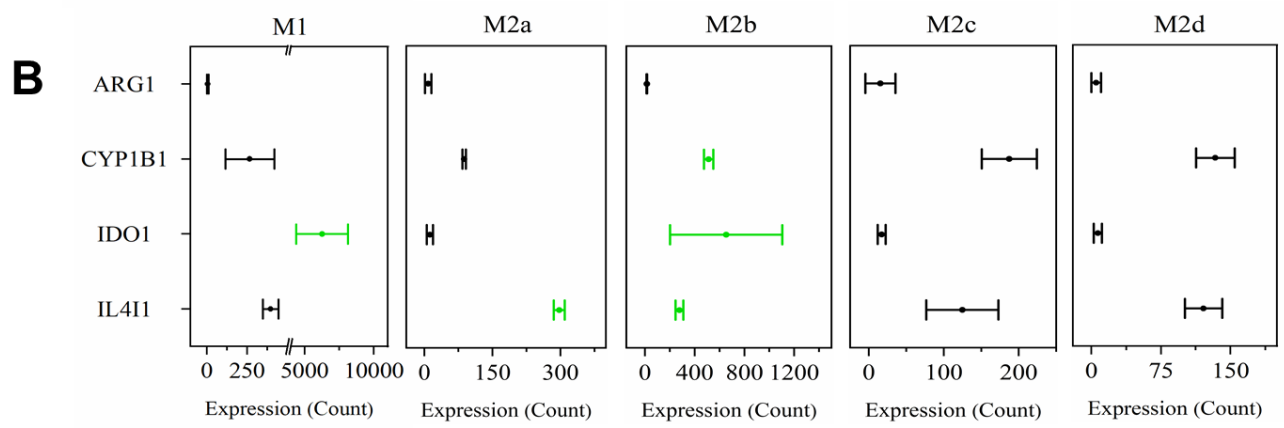

Amino Acid

Metabolism
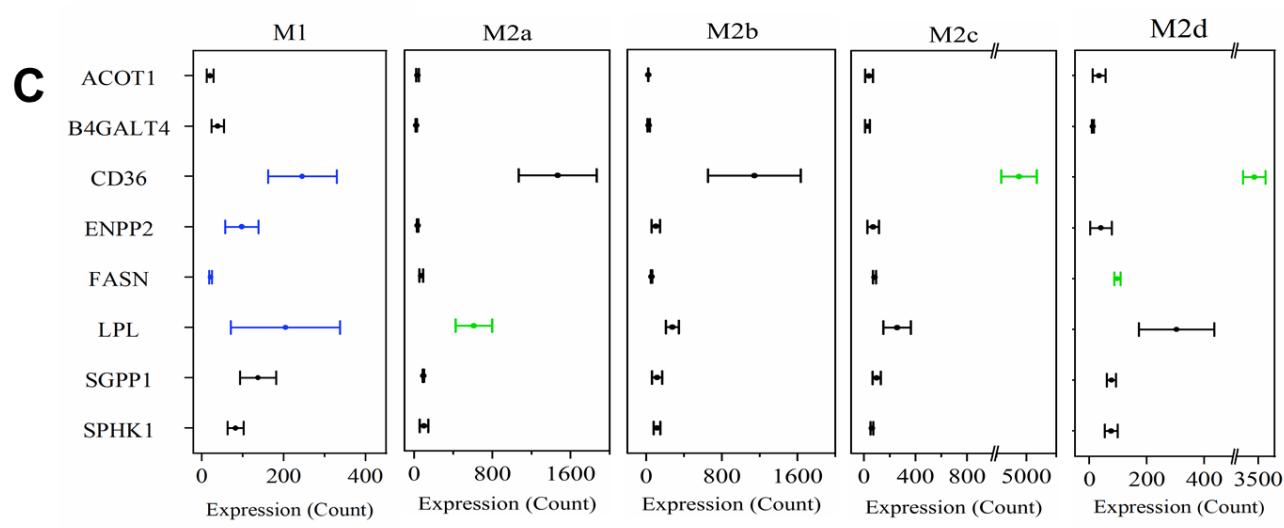

Fatty Acid

Metabolism
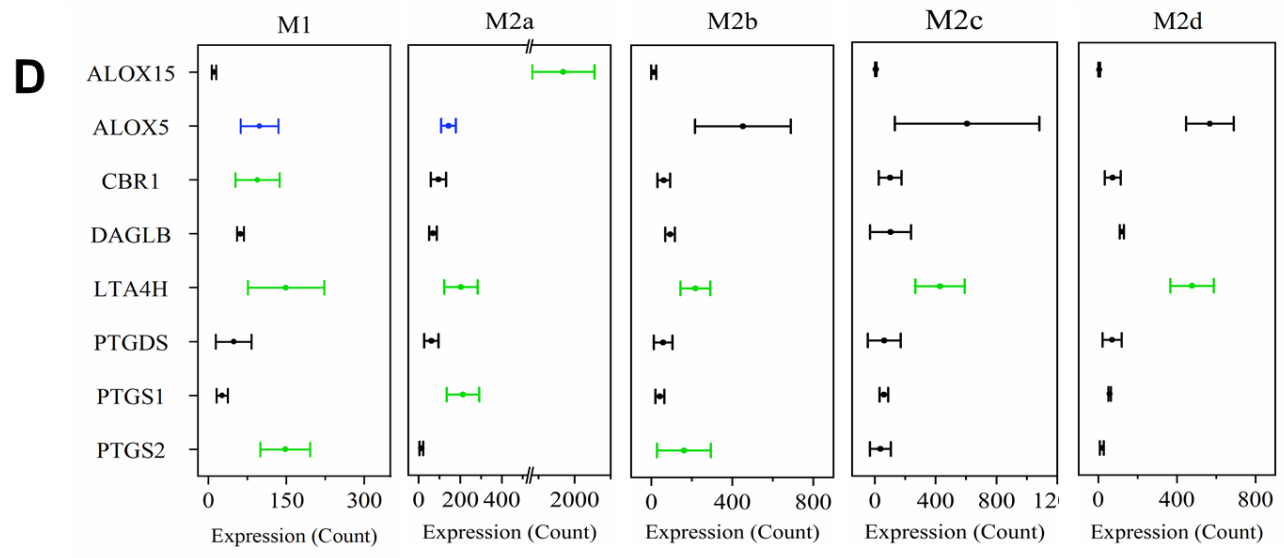

E

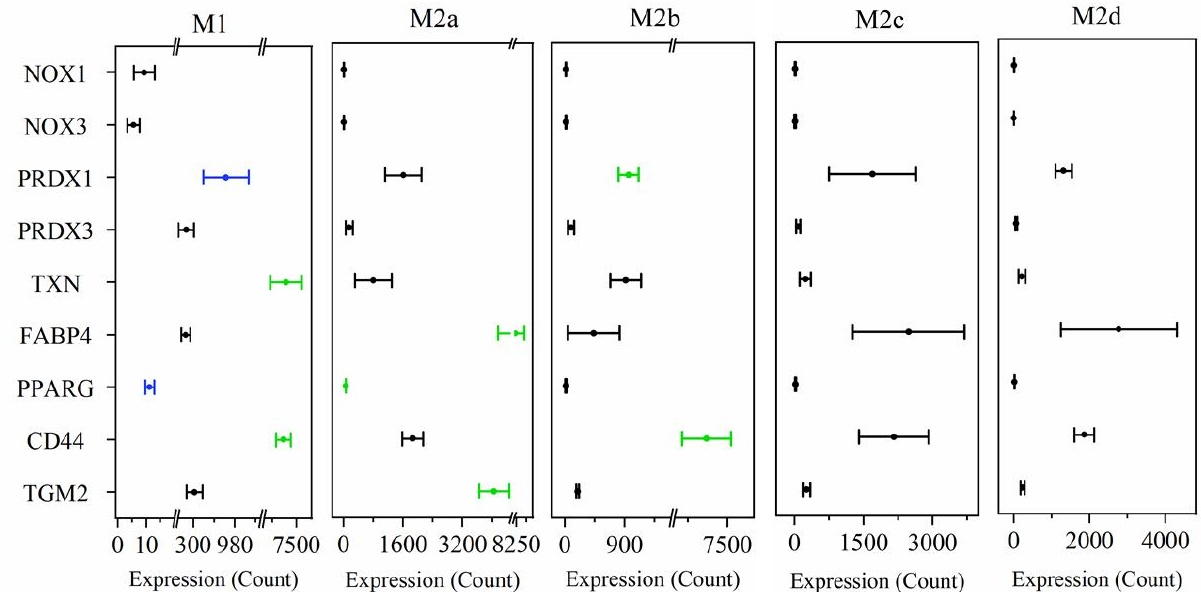

$$
\begin{aligned}
& \begin{array}{l}
\text { Miscellaneous } \\
\text { Pathways } \\
\\
\text { Legend } \\
\text { I Mean } \pm 1.5 \mathrm{SE} \\
\text { - Mean } \\
\log _{2}(\text { Expression })>1 \\
-1>\log _{2} \text { (Expression) }
\end{array}
\end{aligned}
$$


bioRxiv preprint doi: https://doi.org/10.1101/2020.03.10.985788; this version posted November 26, 2020. The copyright holder for this preprint (which was not certified by peer review) is the author/funder. All rights reserved. No reuse allowed without permission.

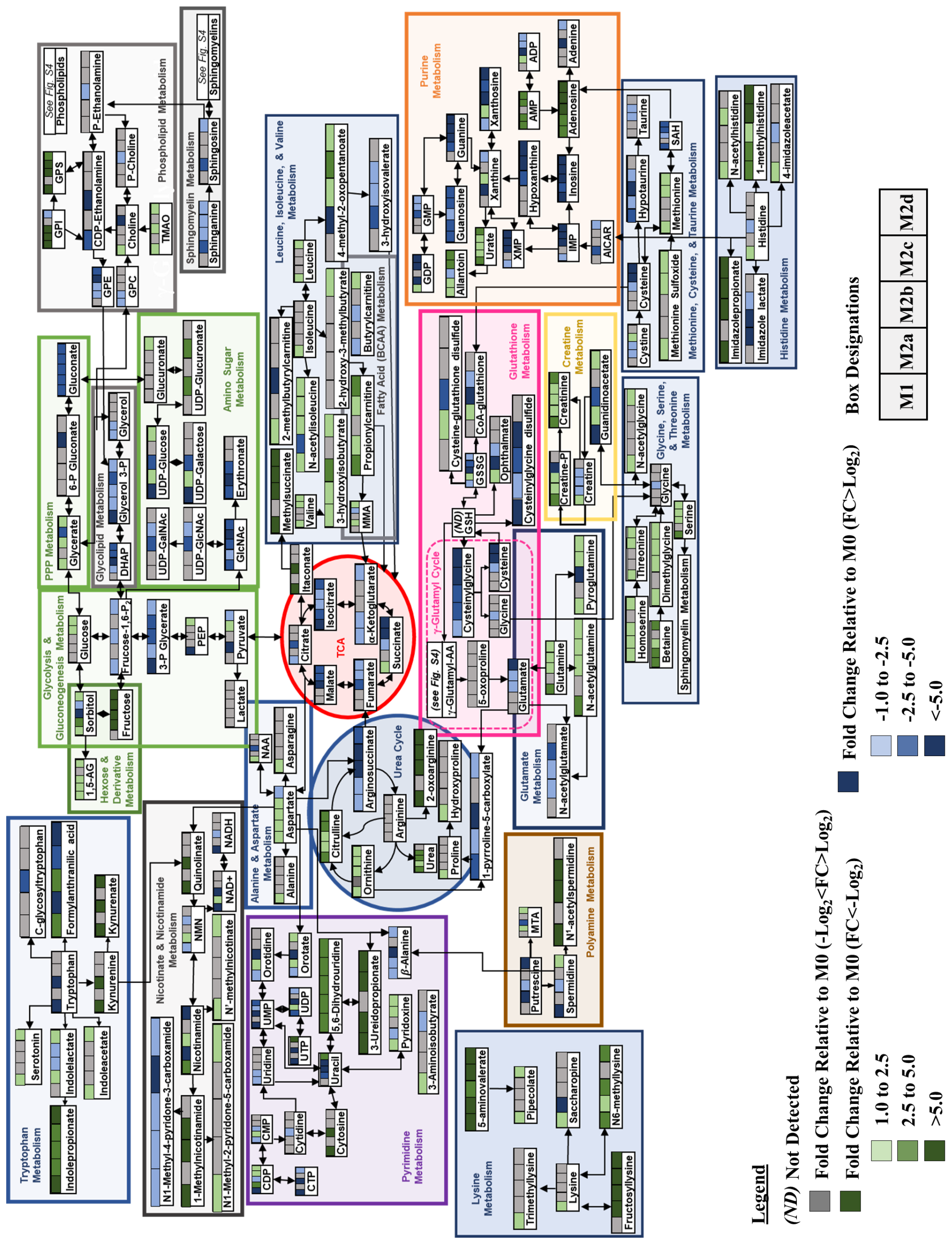




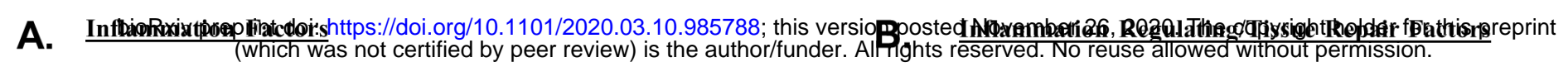
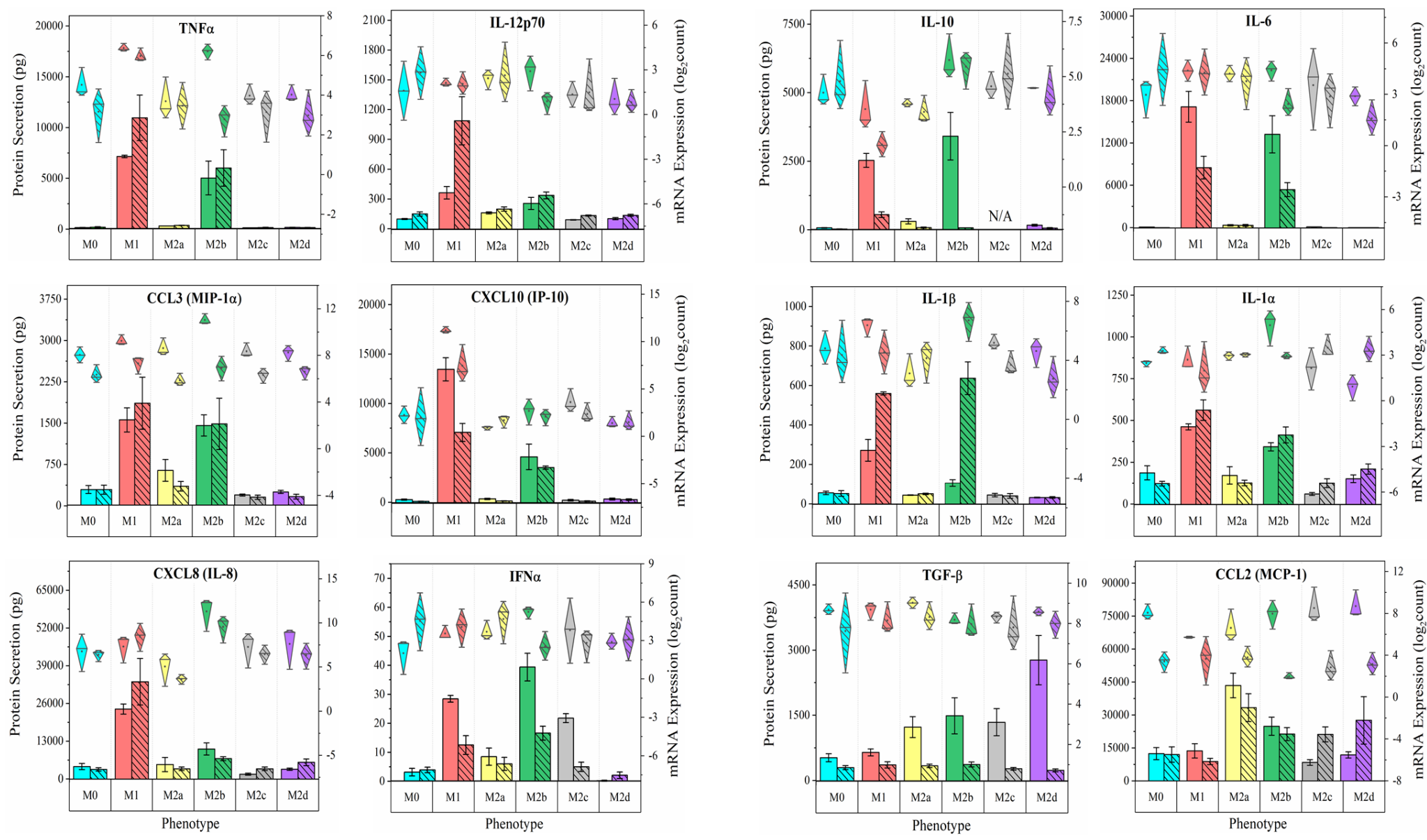

\section{Growth Factors}
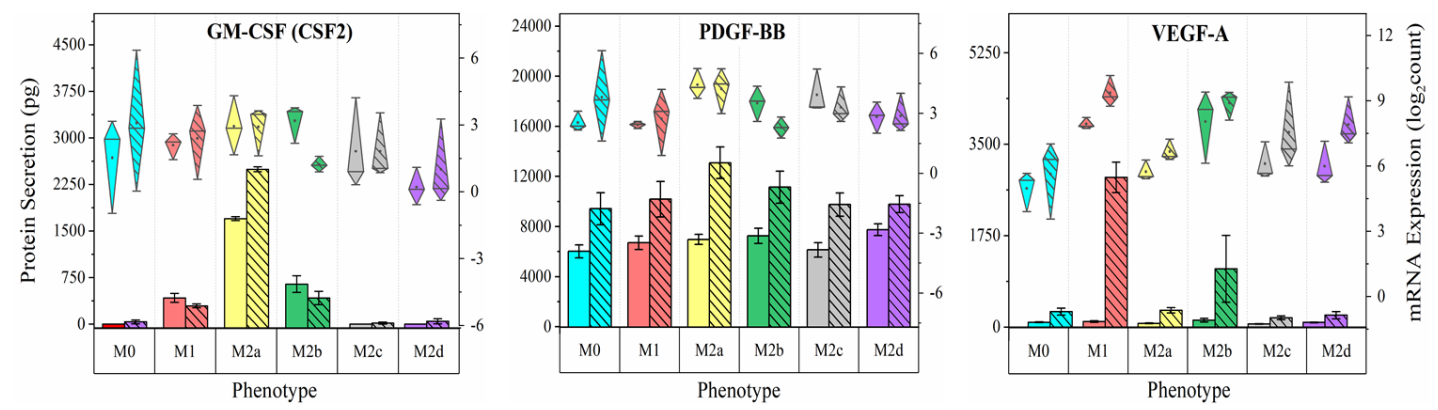

Legend

24 hours

72 hours
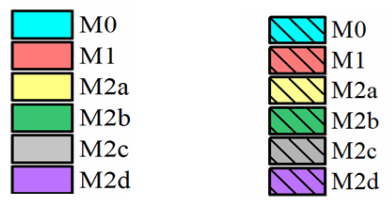

$\bigcirc 25 \% \sim 75 \%$

I Range within 1.5IQR

- Median Line

- Mean 


\section{bioRxiv preprint doi: https://doi.org/10.1101/2020.03.10.985788; this versign posted November 26, 2020. The copyright holder for this preprint (which was not certified by peer review) is the author/funder. A rights reserved. No reuse allowed without permission.}
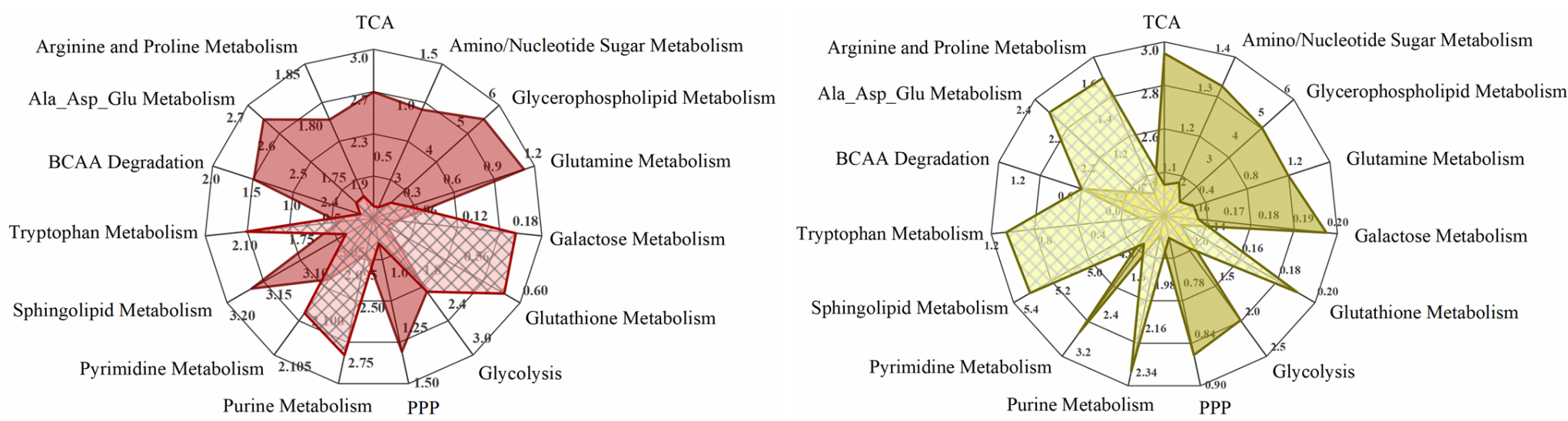

c.

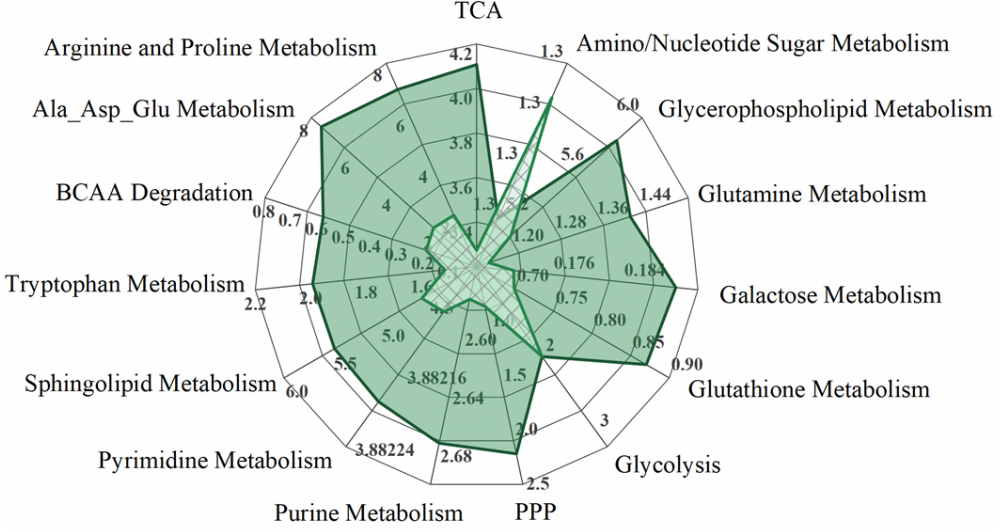

E.

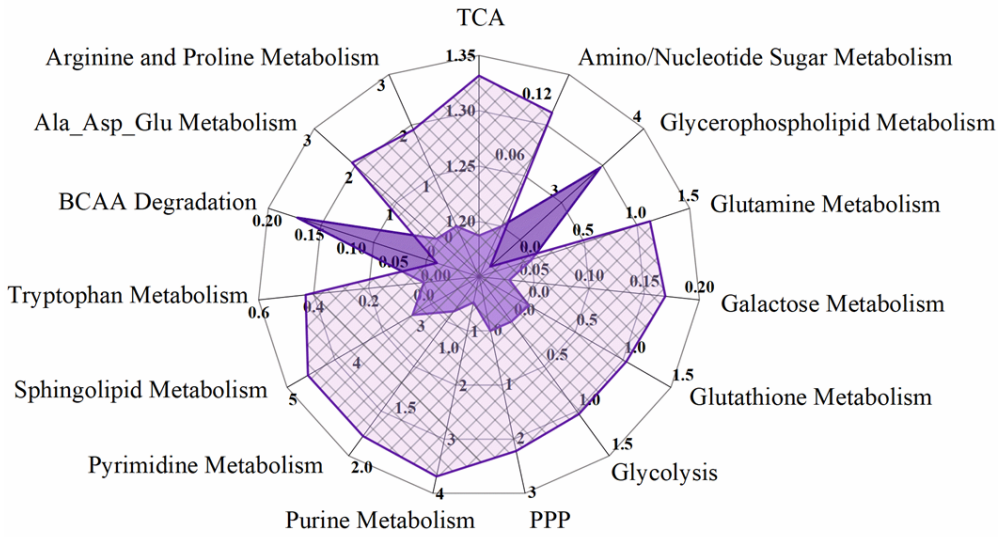

D.

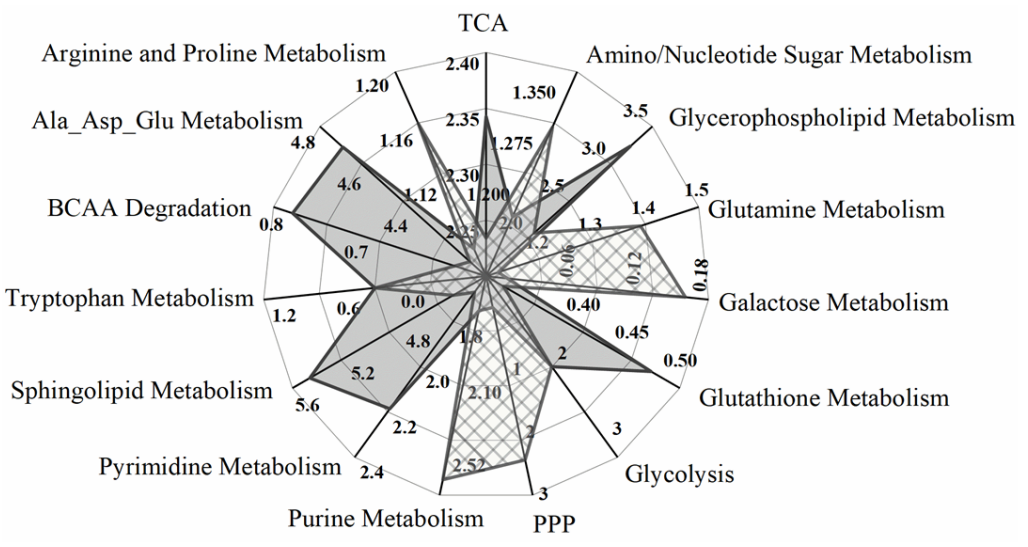

\section{Legend}

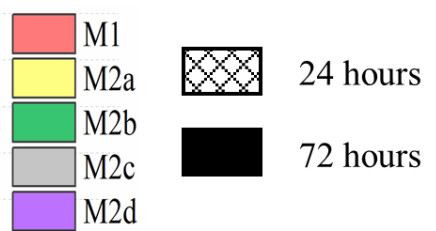



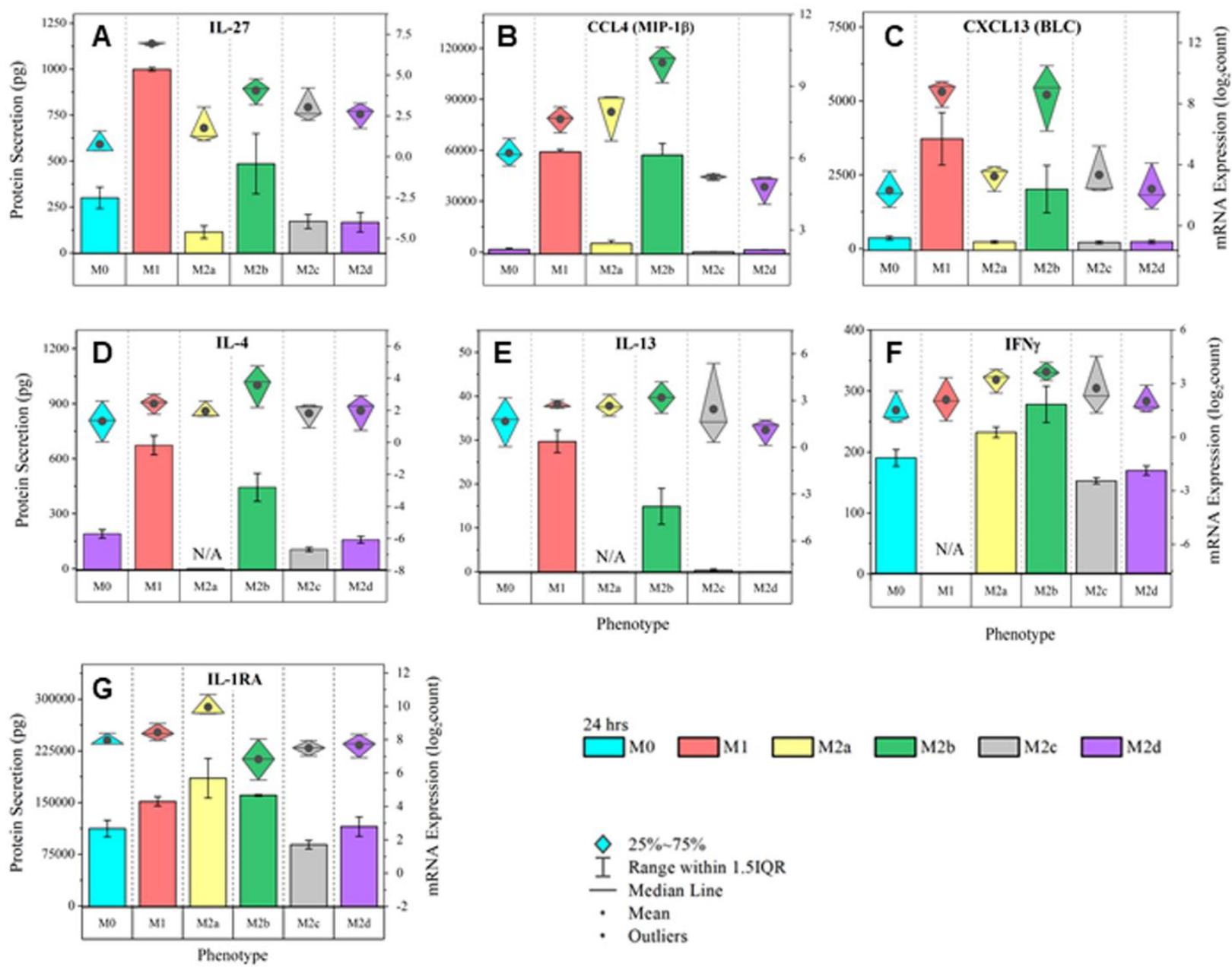

\section{Supplemental Figure 1: MФ Functional Phenotyping by Gene and Protein Expression of}

Immunomodulatory Factors. Multiplex detection of immunomodulatory factors in six M $\Phi$ phenotypes were detected using magnetic bead-based quantification of mRNA and secreted protein. CD14+ monocytes were isolated from human blood-derived PBMCs, differentiated into resting $\mathrm{M} \Phi(\mathrm{M} 0$, shown in blue) with M-CSF ex vivo, and polarized into five activated phenotypes using IFN- $\gamma /$ LPS (M1, shown in red), IL-4/IL-13 (M2a, shown in yellow), IC/LPS (M2b, shown in green), IL-10 (M2c, shown in gray), or IL-6/LIF (M2d, shown in purple). Gene and protein expression profile (diamond-whisker plots and bar charts, respectively) of key functional molecules were profiled by multiplex assay after 24 hours of ex vivo polarization. 
Immunomodulatory factors include IL-27 (A), CCL4 (B), CXCL13 (C) IL-4 (D), IL-13 (E), $\operatorname{IFN} \gamma(\mathbf{F})$, and IL-1RA (G). Diamond-whisker plots display 25\%-75\% quartile range, median, and mean. Bar charts indicate mean \pm SEM. Expression profiles were normalized to total cell count and include three biological replicates $(\mathrm{N}=3)$. 


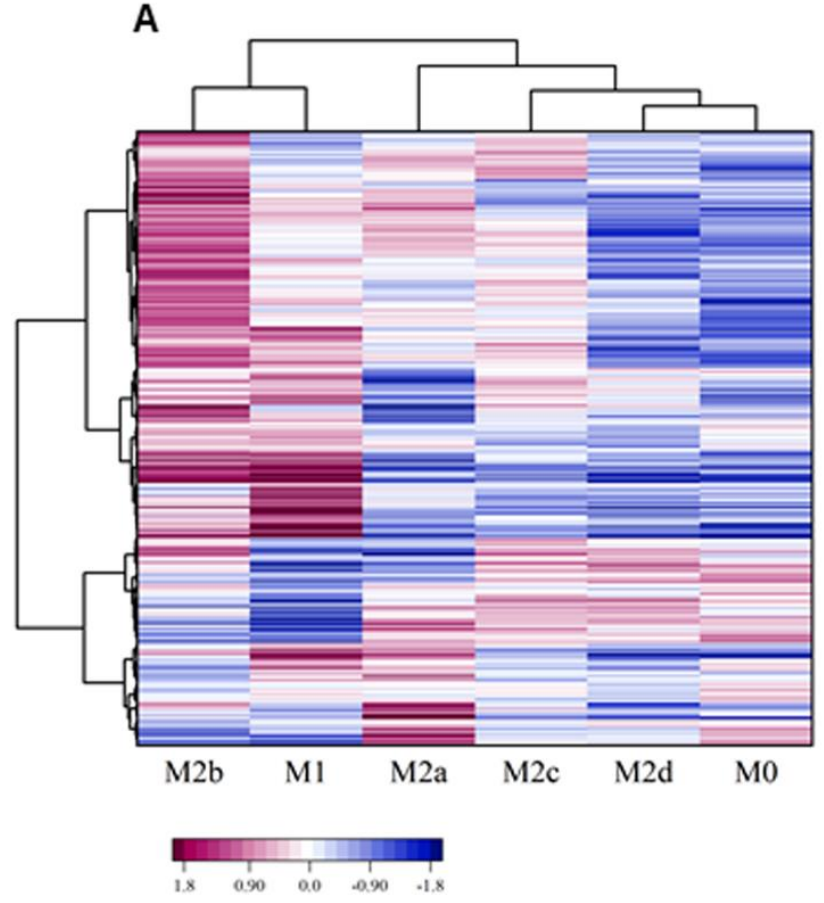

B

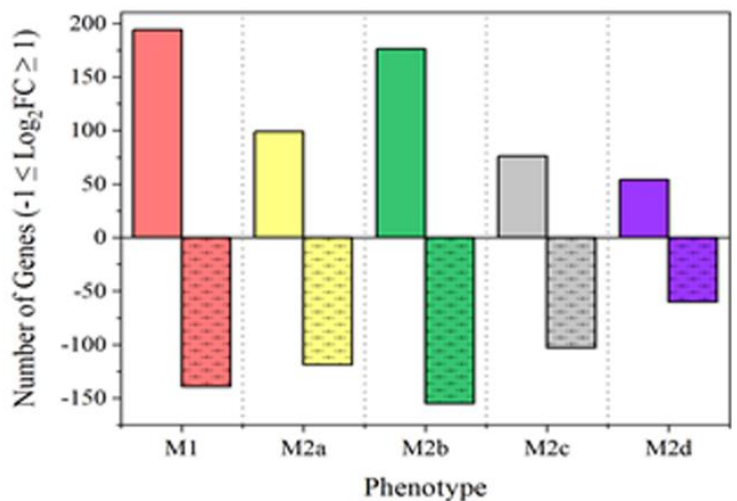

Up Down

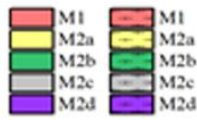

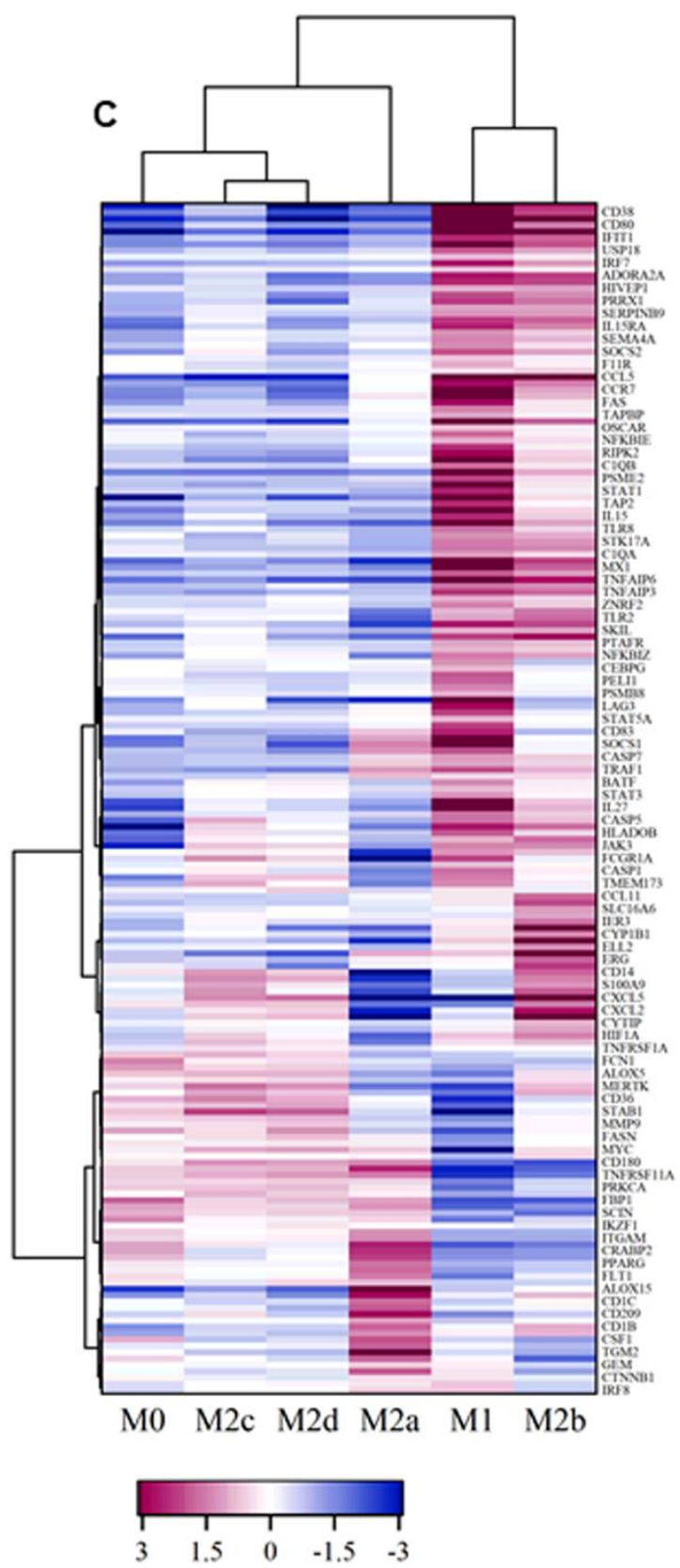




\section{Supplemental Figure 2: Global Transcriptomics Differentiates the MФ Functional}

Phenotypes from the Parent, Resting M0 Phenotype. Hierarchical cluster analysis performed on the normalized expression data identified differences in the transcriptional profiles of the polarized MФ phenotypes relative to the M0 parent MФ. Distance measure is by Pearson correlation with clustering determined using the Ward algorithm. Heatmap scale (-1.8 to 1.8) is colored from blue to red (A). The fold change for each gene was calculated relative to the M0 phenotype and the number of up- and down-regulated genes $\left(-1 \leq \log _{2} \mathrm{FC} \geq 1\right)$ graphed in (B). Gene set enrichment analysis (GSEA) of the global myeloid gene expression revealed 189 significantly differentially expressed genes (SDEGs) identified using a one-way ANOVA with an adjusted p-value (FDR) cutoff of 0.05 . Hierarchical cluster analysis was performed on this data using a distance measure calculated using Pearson correlation with clustering determined using the Ward algorithm. Heatmap scale (-1.8 to 1.8$)$ is colored from blue to red (C). 
A

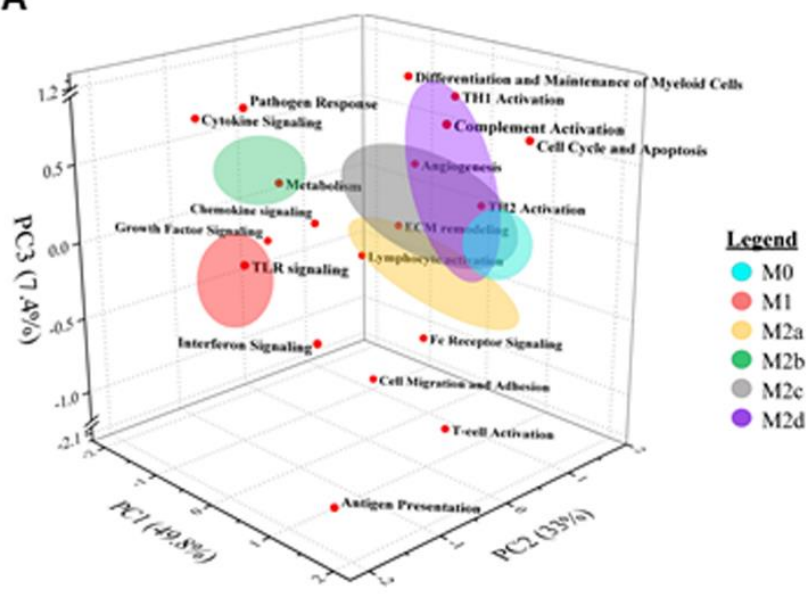

B

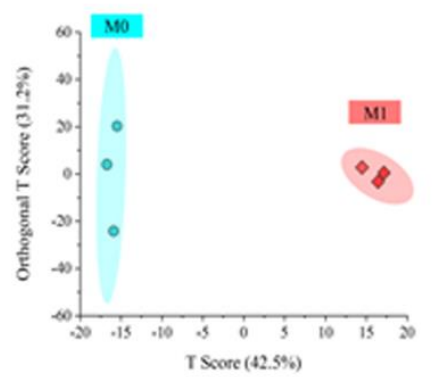

D

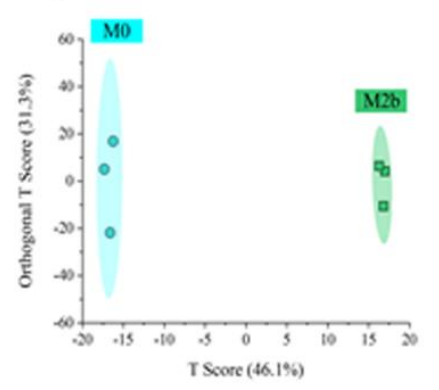

F

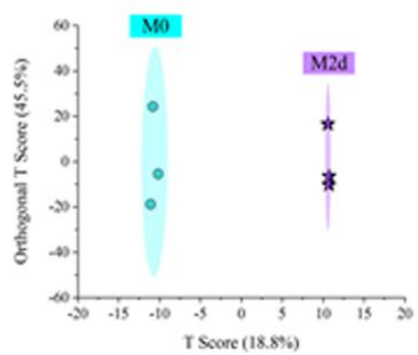

G

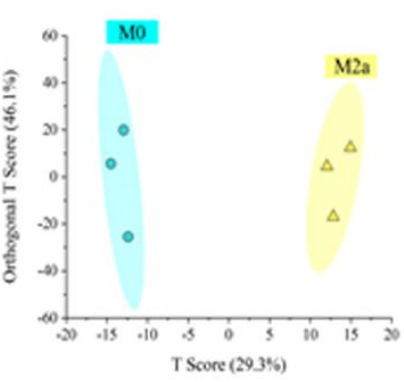

E

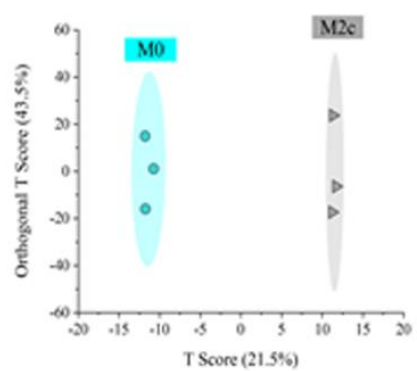

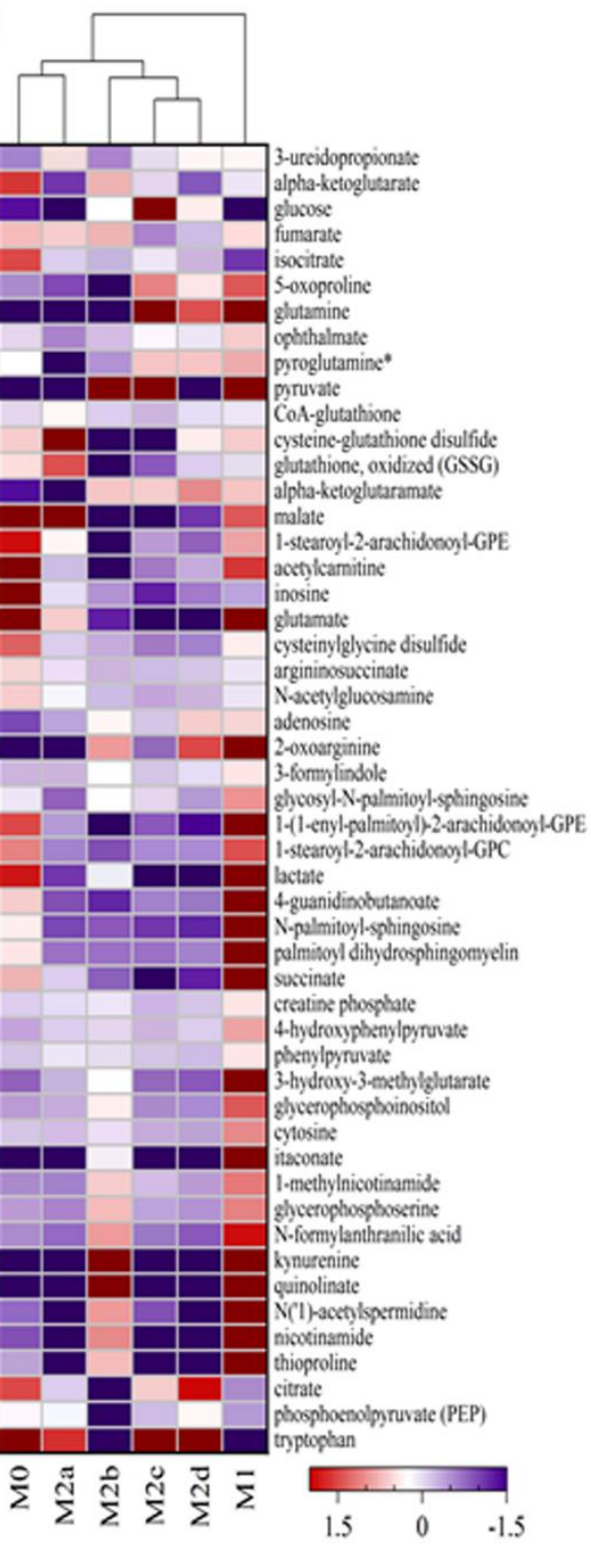




\section{Supplemental Figure 3: МФ Polarization into Functional Phenotypes Activates Distinct Gene Expression Profiles and Metabolic Differences Relative to the Parent, Resting MФ}

Phenotype (M0). Principal component analysis (PCA) 3D loadings plot utilizes red dots to depict the PC1 (x-axis), PC2 (y-axis) and PC3 (z-axis) loadings for each of the 19 Nanostring identified pathways while the colored ellipses denote the 3D representation of the linear combination of the three principal components for each polarized phenotype and the M0 parent cells (A). Orthogonal Projections to Latent Structures Discriminant Analysis (OPLSDA) clustering scores plots with 2D T-scores are depicted for The M0 parent cells (cyan) versus M1 MФs (IFN- $\gamma /$ LPS treated shown in red) (B), M2a MФs (IL-4/IL-13 treated, shown in yellow) (C), M2b MФs (IC/LPS treated, shown in green) (D), M2c MФs (IL-10 treated, shown in gray) (E), and M2d MФs (IL-6/LIF treated, shown in purple) (F). Global metabolomics profile of the parent, resting $\mathrm{M} \Phi$ phenotype (M0) and five polarized MФ phenotypes identified 498 compounds of known identity, normalized to cell count. Hierarchical cluster analysis was performed on the top 50 significantly differentiated metabolites identified using a one-way ANOVA with an adjusted p-value (FDR) cutoff of 0.05 using a distance measure calculated using Pearson correlation with clustering determined using the Ward algorithm. Heatmap scale (1 to 1$)$ is colored from purple to $\operatorname{red}(\mathbf{G})$. 
bioRxiv preprint doi: https://doi.org/10.1101/2020.03.10.985788; this version posted November 26, 2020. The copyright holder for this preprint

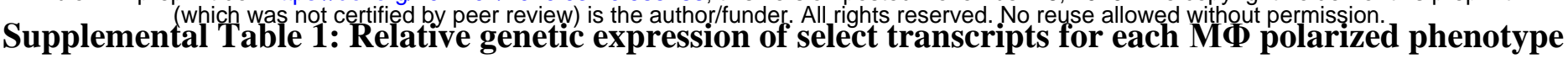

compared to the Parent, Resting МФ Phenotype (M0). Heatmap scale (-8 to 8) is colored from blue to green.

Nanostring-defined pathways associated with specific SDEGs are denoted with gray box with a plus sign.

\begin{tabular}{|c|c|c|c|c|c|c|c|c|c|c|c|c|c|c|c|c|c|c|c|c|c|c|c|c|}
\hline Gene & M1 & M2a & M2b & M2c & M2d & $\begin{array}{l}0 \\
0 \\
0 \\
0 \\
0 \\
.0 \\
.0 \\
0 \\
0 \\
0\end{array}$ & 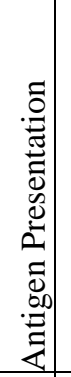 & 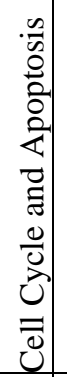 & 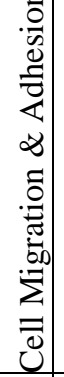 & 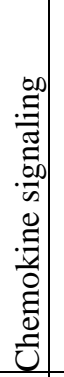 & 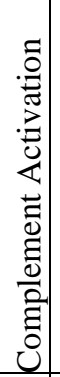 & 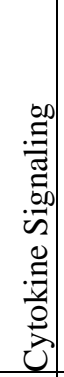 & 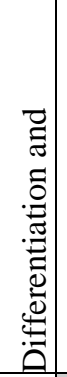 & 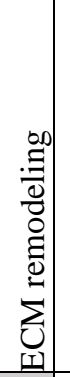 & 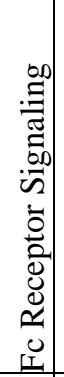 & 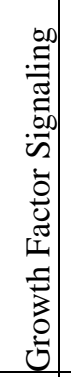 & 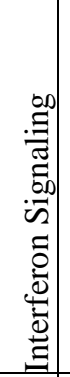 & 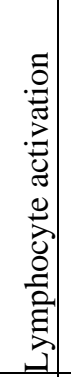 & 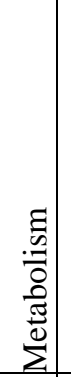 & 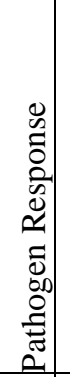 & 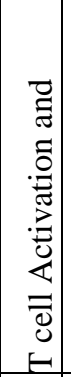 & 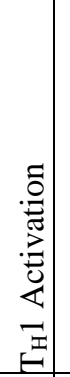 & 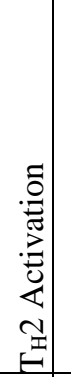 & 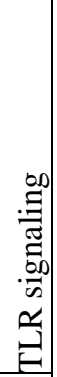 \\
\hline ADAM19 & 6.17 & 4.64 & 4.92 & 0.74 & 1.42 & & & & & & & & & + & & & & & & & & & & \\
\hline ALOX15 & 0.74 & 10.70 & 0.74 & 0.00 & 0.00 & & & & & & & & & & & & & & & & & & & \\
\hline CCL1 & 5.32 & 0.00 & 3.17 & 0.00 & 0.00 & & & & & + & & & & & & & & & & & & & & \\
\hline CCL17 & -0.42 & 9.06 & 0.32 & -0.42 & -0.42 & & & & & + & & & & & & & & & & & & & & \\
\hline CCL18 & 3.53 & 3.77 & 2.44 & 1.23 & -1.48 & & & & & + & & & & & & & & & & & & & & \\
\hline CCL18 & 3.53 & 3.77 & 2.44 & 1.23 & -1.48 & & & & & + & & & & & & & & & + & & & & & \\
\hline CCL22 & -2.02 & 2.71 & -1.52 & -1.86 & -1.73 & & & & & + & & & & & & & & & & & & & & \\
\hline CCL4 & 1.46 & 1.93 & 3.92 & -1.76 & -1.57 & & & & & + & & & & & & & & & & + & & & & + \\
\hline CCL5 & 6.33 & 2.54 & 5.29 & -1.99 & -1.36 & & & & & & & + & & & & & & & & + & & & & + \\
\hline CCL8 & 6.51 & 0.69 & 3.21 & 2.05 & 2.49 & & & & & + & & & & & & & & & & & & & & \\
\hline CD163 & -0.32 & -2.51 & 1.82 & 1.67 & 1.26 & & & & & & & & + & & & & & & & & & & & \\
\hline CD209 & -2.95 & 4.23 & -0.78 & 0.80 & -0.05 & & + & & & & & & & & & & & + & & & & & & \\
\hline CD36 & -3.55 & -0.92 & -1.30 & 0.66 & 0.18 & & + & & & & & & & + & & & & & + & & & & & + \\
\hline CD40 & 3.14 & 1.20 & 0.93 & 0.32 & 0.45 & & & & + & & & & & & & & & + & & & & + & & + \\
\hline CD86 & 1.28 & 0.43 & -0.63 & -1.38 & -0.97 & & & & + & & & & & & + & + & & + & & & + & & & + \\
\hline CXCL1 & -1.42 & -4.58 & 4.06 & -1.17 & -0.42 & & & & & + & & + & & & & & & & & + & & & & \\
\hline CXCL11 & 8.66 & 0.42 & 1.22 & 0.00 & 0.00 & & & & & + & & & & & & & & & & & & & & + \\
\hline CXCL2 & -0.18 & -5.56 & 3.16 & 0.45 & 1.15 & & & & & + & & + & & & & & & & & + & & & & \\
\hline CXCL3 & -0.77 & -6.38 & 3.91 & 0.51 & 1.14 & & & & & + & & + & & & & & & & & & & & & \\
\hline CXCL5 & -5.06 & -8.43 & 4.72 & 0.35 & 0.77 & & & & & + & & + & & & & & & & & & & & & \\
\hline CXCL8 & 0.16 & -2.22 & 4.35 & 0.30 & 0.93 & & & & & + & & & & & & & & & & + & & & & + \\
\hline CXCL9 & 12.30 & 0.74 & 4.09 & 2.66 & 2.12 & & & & & + & & & & & & & & & & & & & & + \\
\hline DUSP1 & 3.01 & 1.15 & 3.84 & 2.45 & 2.38 & + & & & & & & + & & & + & + & & + & & & & & & \\
\hline ENPP2 & 4.71 & 2.81 & 4.55 & 4.17 & 3.38 & & & & & & & & & & & & & & + & & & & & \\
\hline FABP4 & -3.61 & 1.95 & -2.30 & 0.28 & 0.45 & & & & & & & & & & & & & & + & & & & & \\
\hline HIF1A & 1.45 & -1.40 & 2.27 & 1.51 & 1.02 & & & & & & & & & & & + & & & & & & & & \\
\hline ID1 & 0.00 & 0.00 & 2.81 & 0.00 & 2.00 & + & & + & & & & & & & & & & & & & & & & \\
\hline IER3 & 1.24 & 0.50 & 2.56 & 1.44 & 0.58 & & & & & & & & & & & & & + & & & & & & \\
\hline IRF1 & 5.73 & 0.30 & 2.42 & 0.62 & 1.27 & & & & & & & & & & & & + & & & & & & & \\
\hline IRF7 & 4.81 & 0.06 & 3.25 & 0.13 & 0.67 & & & & & & & & & & & & + & & & + & & & & + \\
\hline IRF8 & 1.24 & 1.12 & -0.54 & 0.43 & 0.49 & & & & & & & & & & & & + & & & & & & & \\
\hline IRG1 & 11.00 & 0.00 & 7.15 & 1.22 & 0.00 & & & & & & & & & & & & & & + & + & & & & \\
\hline ISG15 & 5.53 & 0.15 & 3.74 & -0.97 & -0.24 & & & & & & & & & & & & + & & & + & & & & \\
\hline MERTK & -3.98 & -2.71 & 0.15 & 1.05 & 0.74 & & & & & & & & & & & & & & & & & & & \\
\hline MMP12 & -2.50 & 2.01 & -1.13 & 1.73 & 0.91 & & & & & & & & & & & & & & & & & & & \\
\hline MRC1 & -3.98 & 1.24 & -3.96 & -1.12 & -1.63 & & & & & & & & & & & & & & & & & & & \\
\hline PPARG & -3.24 & 1.30 & -2.72 & -1.96 & -0.72 & & & & & & & & & & & & & & + & & & & & \\
\hline PSME2 & 2.91 & 0.26 & 1.03 & -0.01 & -0.08 & + & + & & & & & + & & & + & + & + & + & + & & & & & \\
\hline PTGS1 & -1.97 & 1.62 & -1.81 & -0.73 & -0.33 & & & & & & & & & & & & & & + & & & & & \\
\hline PTGS2 & 4.44 & -1.08 & 4.44 & 1.34 & 0.962 & & & & & & & + & & & & & & & + & & & & & \\
\hline S100A8 & -0.07 & -2.82 & 2.20 & 1.45 & 0.16 & & & & & & & & + & & & & & & & & & & & \\
\hline S100A9 & -0.88 & -2.01 & 1.17 & 0.96 & 0.25 & & & & & & & & + & & & & & & & & & & & \\
\hline SOCS3 & 6.30 & 1.26 & 5.24 & 4.01 & 3.65 & & + & & & & & + & & & & + & + & & & & & & & \\
\hline SPHK1 & 1.50 & 1.77 & 1.82 & 0.71 & 1.46 & + & & & & & & & & & & & & & + & & & & & \\
\hline STAT1 & 3.26 & -0.37 & 0.92 & -0.03 & 0.07 & & & & & + & & + & & & & + & + & & & & & + & & + \\
\hline STAT3 & 2.06 & 0.05 & 0.82 & 0.70 & 0.82 & & & & & + & & + & & & & + & & & & & & & & \\
\hline TGM2 & -0.38 & 3.33 & -1.24 & -0.74 & -0.83 & & & & & & & & & & & & & & + & & & & & \\
\hline
\end{tabular}


bioRxiv preprint doi: https://doi.org/10.1101/2020.03.10.985788; this version posted November 26, 2020. The copyright holder for this preprint (which was not certified by peer review) is the author/funder. All rights reserved. No reuse allowed without permission. 
A

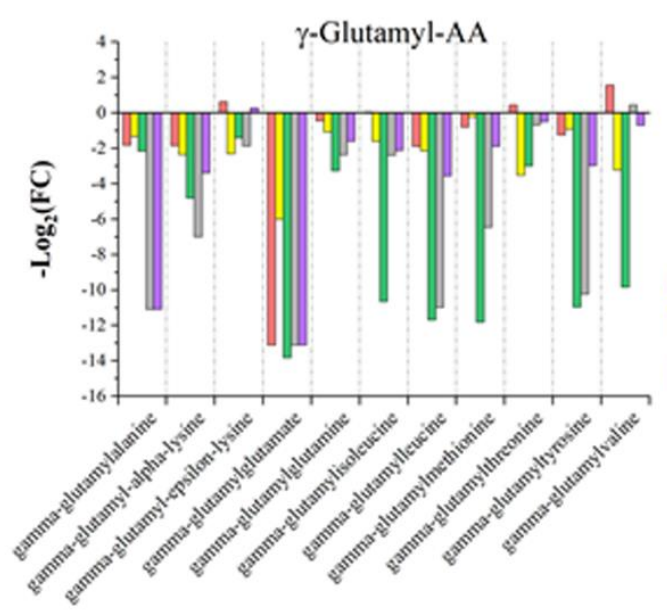

c
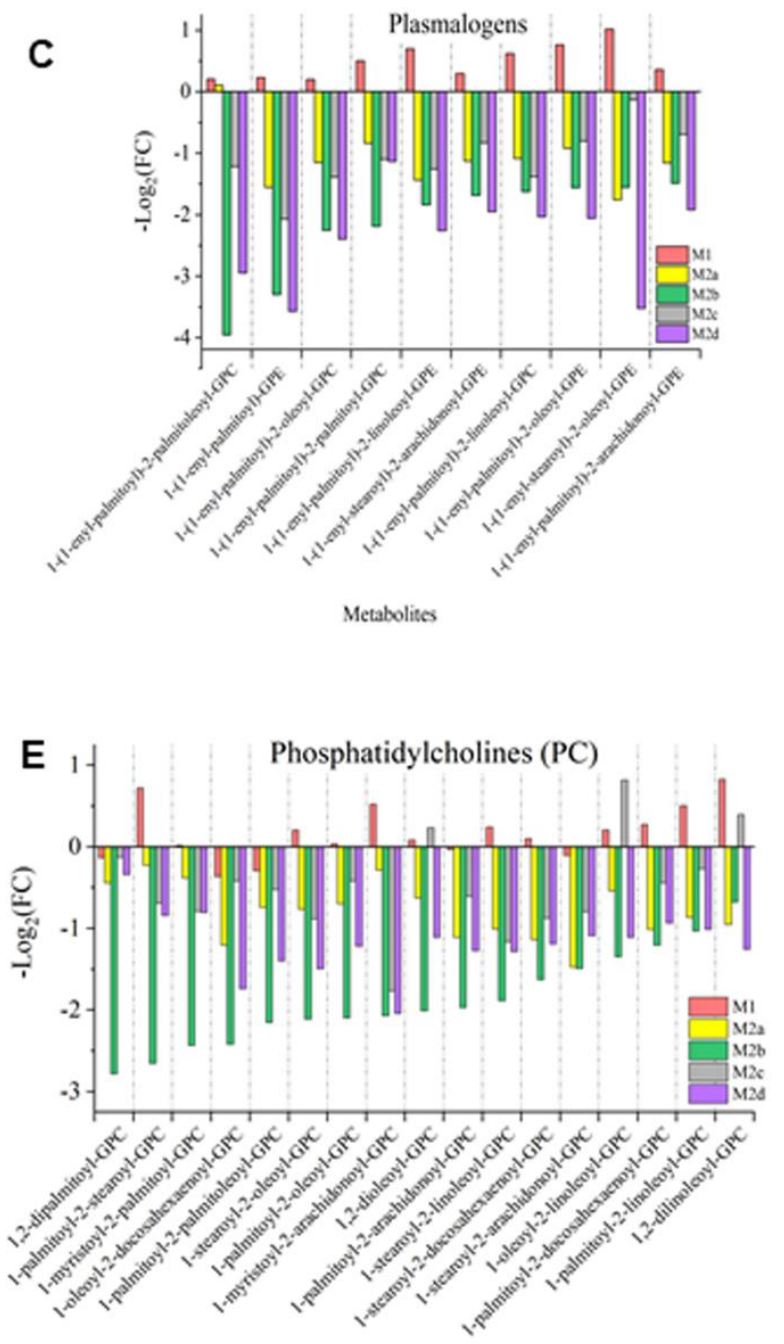

Metabolites

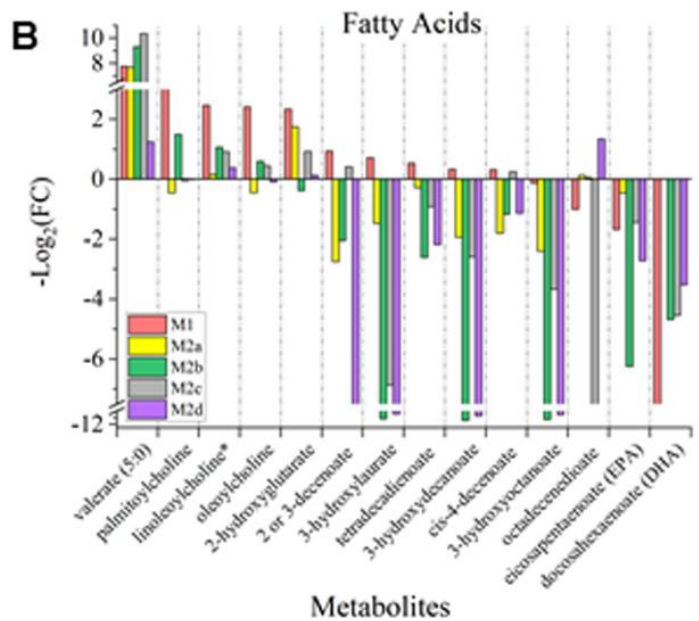

Metabolites
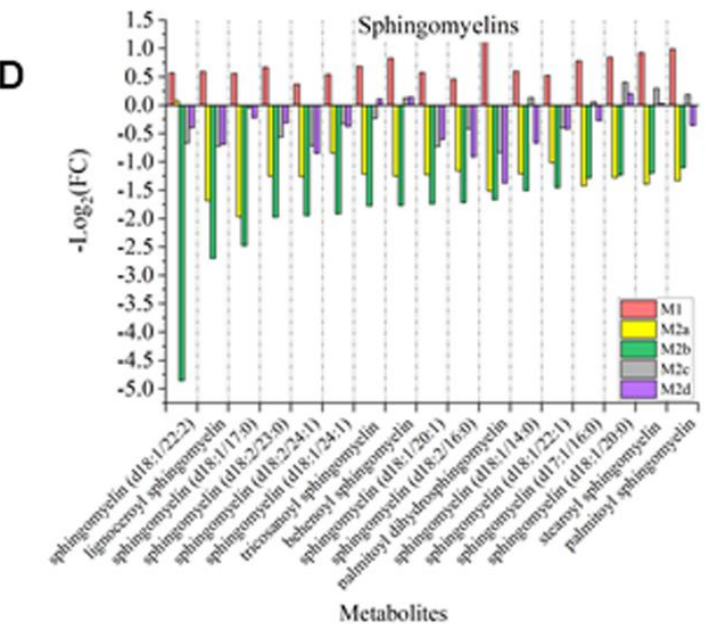

F

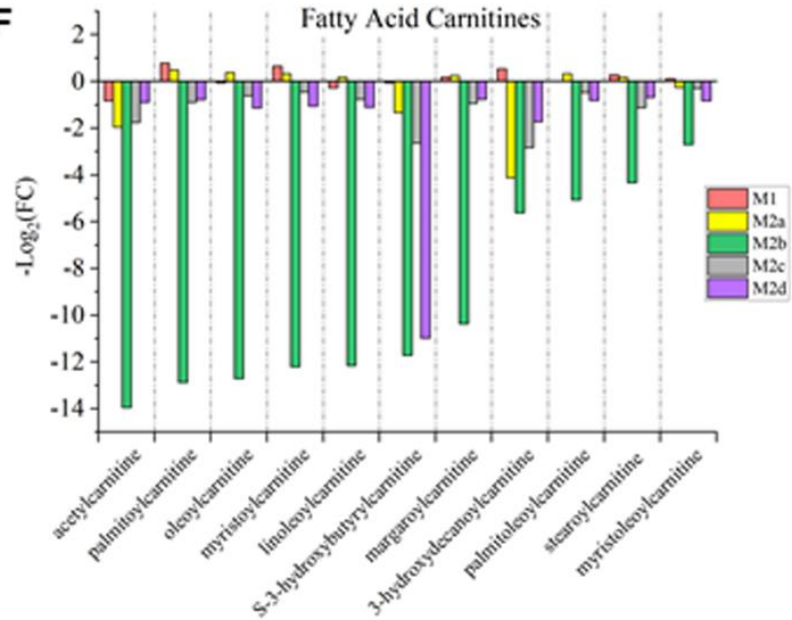

Metabolite 


\section{Supplemental Figure 4: Untargeted Metabolomics of Five Polarized MФ Phenotypes}

\section{Identifies Phenotypic Differences in $\gamma$-Glutamyl Amino Acid Metabolism and Lipid}

Metabolism Relative to the Parent, Resting МФ Phenotype. Histogram plots depict the accumulation of (positive $-\log _{2} \mathrm{FC}$ bars) or decrease of (negative $-\log _{2} \mathrm{FC}$ bars) lipid metabolites for the M1 MФs (IFN- $\gamma /$ LPS treated, shown in red), M2a MФs (IL-4/IL-13 treated, shown in yellow), M2b MФs (IC/LPS treated, shown in green), M2c MФs (IL-10 treated, shown in gray), and M2d MФs (IL-6/LIF treated, shown in purple) relative to the M0 parent cells. The represented metabolic classifications include $\gamma$-glutamyl amino acids (A), fatty acids (B), plamologens (C), sphingomyelins (D), phosphotidylcholines (PC) (E), and fatty acid carnitines (F). 
Polarized Phenotype Compared to the Parent, Resting МФ Phenotype (M0). Pathway

categories and metabolites correspond to the data pictures in Figure 9. Heatmap scale (-8 to 8) is colored from blue to green.

\begin{tabular}{|c|c|c|c|c|c|c|}
\hline \multirow{2}{*}{ Metabolite } & \multicolumn{5}{|c|}{ Phenotype } & \multirow{2}{*}{$\begin{array}{c}\text { Metabolism } \\
\text { Pathway }\end{array}$} \\
\hline & M1 & M2a & M2b & M2c & M2d & \\
\hline erythronate & -3.74 & -12.54 & -4.04 & -0.08 & 0.58 & \multirow{8}{*}{ Amino Sugar } \\
\hline $\mathrm{N}$-acetylglucosamine (GlcNAc) & -2.92 & -1.13 & -4.73 & -11.22 & -3.82 & \\
\hline glucuronate & 0.15 & 0.42 & 0.69 & 0.17 & 0.97 & \\
\hline UDP-Galactose & -5.44 & 1.71 & -3.44 & 0.15 & -0.27 & \\
\hline UDP-GalNAc & 0.11 & 0.38 & -2.68 & -1.21 & -0.33 & \\
\hline UDP-GlcNAc & 0.11 & 0.38 & -2.68 & -1.21 & -0.33 & \\
\hline UDP-Glucose & -9.38 & 1.25 & -2.95 & -0.48 & 2.12 & \\
\hline UDP-Glucuronate & 0.02 & 3.64 & -0.55 & 2.61 & -0.66 & \\
\hline alanine & 1.10 & 0.02 & 0.30 & 0.21 & 0.43 & \multirow{4}{*}{$\begin{array}{c}\text { Alanine and } \\
\text { Aspartate }\end{array}$} \\
\hline asparigine & 1.40 & 0.20 & -0.21 & 0.59 & 0.40 & \\
\hline aspartate & 1.47 & 1.02 & 1.79 & 1.11 & -1.72 & \\
\hline N-acetylaspartate (NAA) & 1.04 & 0.37 & -2.82 & -0.08 & 0.25 & \\
\hline creatine & 1.51 & -1.75 & -2.08 & -1.11 & -0.92 & \multirow{4}{*}{ Creatine } \\
\hline creatinine & 2.89 & 1.50 & 1.28 & 1.78 & 2.85 & \\
\hline creatine phosphate & 3.83 & 1.88 & 2.34 & -6.66 & -0.20 & \\
\hline guanidinoacetate & -10.10 & 1.82 & -2.63 & 0.56 & 1.10 & \\
\hline butylcarnitine & -1.33 & -0.31 & -1.22 & 0.55 & 1.08 & \multirow{3}{*}{$\begin{array}{l}\text { Fatty Acid } \\
\text { (BCAA) }\end{array}$} \\
\hline methylmalonate (MMA) & -0.75 & 2.07 & -0.07 & -1.80 & 1.12 & \\
\hline propionylcarnitine & 2.97 & 1.93 & -0.05 & 2.31 & 2.57 & \\
\hline glutamate & -0.14 & -0.59 & -2.18 & -2.33 & -15.29 & \multirow{5}{*}{ Glutamate } \\
\hline glutamine & 2.76 & -0.39 & 1.51 & 2.09 & 2.07 & \\
\hline $\mathrm{N}$-acetylglutamate & 0.12 & -0.98 & -1.94 & -2.68 & -0.42 & \\
\hline $\mathrm{N}$-acetylglutamine & 3.15 & 0.19 & 2.42 & 2.26 & 2.17 & \\
\hline pyroglutamine & 2.43 & -0.44 & -10.24 & 2.16 & 2.11 & \\
\hline 5-oxoproline & 0.56 & 0.17 & -0.21 & 0.43 & 0.39 & \multirow{9}{*}{ Glutathione } \\
\hline CoA-glutathione & 0.06 & 1.69 & -9.08 & -1.41 & -0.41 & \\
\hline cysteine & -0.05 & -0.03 & -1.34 & -12.87 & -12.87 & \\
\hline cysteine-glutathione disulfide & 0.28 & 1.80 & -3.11 & -0.33 & 0.50 & \\
\hline cysteinylglycine & -1.51 & -2.59 & -3.83 & -12.22 & -12.22 & \\
\hline cysteinylglycine disulfide & -12.57 & -1.00 & -3.70 & -7.01 & -0.76 & \\
\hline glutathione, oxidized (GSSG) & -1.52 & 1.29 & -12.04 & -3.89 & -1.42 & \\
\hline glycine & 0.79 & -0.26 & -1.52 & -0.19 & -0.26 & \\
\hline ophthalmate & 2.06 & -8.95 & -1.38 & 0.60 & -0.01 & \\
\hline
\end{tabular}


Polarized Phenotype Compared to the Parent, Resting МФ Phenotype (M0). Pathway

categories and metabolites correspond to the data pictures in Figure 9. Heatmap scale (-8 to 8$)$ is colored from blue to green.

\begin{tabular}{|c|c|c|c|c|c|c|}
\hline betaine & 4.49 & 4.05 & 1.04 & -0.16 & 3.41 & \multirow{7}{*}{$\begin{array}{l}\text { Glycine, Serine } \\
\text { and Threonine }\end{array}$} \\
\hline dimetylglycine & 1.83 & -0.10 & 1.18 & 1.29 & 1.64 & \\
\hline glycine & 0.79 & -0.26 & -1.52 & -0.19 & -0.26 & \\
\hline homoserine & 1.11 & 2.18 & 1.41 & 0.94 & 1.73 & \\
\hline $\mathrm{N}$-acetylglycine & 1.24 & 0.12 & -0.56 & 0.56 & 2.37 & \\
\hline serine & 2.66 & -0.93 & 2.11 & 1.88 & 1.74 & \\
\hline threonine & 2.42 & -0.03 & 0.85 & 1.69 & 1.23 & \\
\hline $\begin{array}{l}\text { dihydroxyacetone phosphate } \\
\text { (DHAP) }\end{array}$ & -0.13 & -1.67 & -12.42 & -2.58 & -2.45 & \multirow{3}{*}{ Glycolipid } \\
\hline glycerol & -1.11 & -1.09 & -0.10 & -0.61 & 0.95 & \\
\hline glycerol 3-phosphate & -11.27 & -1.27 & -1.88 & -2.89 & -1.89 & \\
\hline 3-phosphoglycerate & -12.79 & -1.50 & -2.90 & -3.78 & -2.05 & \multirow{6}{*}{$\begin{array}{l}\text { Glycolysis and } \\
\text { Gluconeogenesis }\end{array}$} \\
\hline frucose-1,6-diphosphate & -1.31 & -0.89 & -2.38 & -0.88 & -1.34 & \\
\hline glucose & 0.80 & 0.60 & 0.76 & 2.23 & 0.95 & \\
\hline lactate & 0.81 & -0.06 & -0.36 & -0.07 & -0.06 & \\
\hline phosphoenolpyruvate (PEP) & -10.01 & 0.05 & -0.06 & -0.08 & 1.11 & \\
\hline pyruvate & -0.66 & -14.22 & 0.40 & 0.98 & -1.83 & \\
\hline 1,5-anhydroglucitol (AG) & 1.82 & 1.23 & 1.41 & 0.94 & 2.21 & \multirow{3}{*}{$\begin{array}{l}\text { Hexose and } \\
\text { Derivatives }\end{array}$} \\
\hline fructose & 0.00 & 9.02 & 10.96 & 11.88 & 11.44 & \\
\hline sorbitol & 1.62 & 2.71 & -8.59 & -1.25 & 2.31 & \\
\hline 1-methylhistidine & 2.96 & 3.65 & 1.31 & 5.60 & 5.89 & \multirow{6}{*}{ Histidine } \\
\hline 4-imidazoleacetate & 1.57 & 0.38 & -2.13 & 0.69 & 0.31 & \\
\hline histidine & 1.54 & -0.29 & -1.25 & 0.80 & -0.02 & \\
\hline imidazole propionate & 8.63 & 0.00 & 9.03 & 8.68 & 9.30 & \\
\hline imidizole lactate & -0.90 & -9.48 & -10.21 & -1.25 & -0.38 & \\
\hline $\mathrm{N}$-acetylhistidine & 1.96 & -0.72 & 0.72 & 1.39 & 0.67 & \\
\hline 2-hydroxy-3-methylbutyrate & 0.86 & 0.43 & 0.90 & -0.12 & 1.41 & \multirow{9}{*}{$\begin{array}{c}\text { Leucine, } \\
\text { Isoleucine and } \\
\text { Valine }\end{array}$} \\
\hline 2-methylbutyrylcarnitine & 1.70 & -0.25 & -9.29 & -0.70 & -0.28 & \\
\hline 2-oxo-isovalerate & 6.01 & 3.63 & 5.68 & 7.06 & 6.51 & \\
\hline 3-hydroxyisobutyrate & 1.61 & 1.40 & 2.69 & 0.68 & 2.27 & \\
\hline 3-hydroxyisovalerate & 0.49 & -3.65 & -1.50 & -1.19 & 0.78 & \\
\hline 3-methyl-2-oxovalerate & 0.28 & -0.90 & 1.24 & 2.57 & 2.05 & \\
\hline 4-methyl-2-oxopentanoate & 0.45 & -9.71 & 1.58 & 2.91 & 2.38 & \\
\hline isoleucine & 1.17 & 0.30 & 0.04 & 0.40 & 0.62 & \\
\hline leucine & 1.53 & 0.34 & 0.30 & 0.95 & 1.06 & \\
\hline
\end{tabular}


Polarized Phenotype Compared to the Parent, Resting МФ Phenotype (M0). Pathway

categories and metabolites correspond to the data pictures in Figure 9. Heatmap scale (-8 to 8$)$ is colored from blue to green.

\begin{tabular}{|c|c|c|c|c|c|c|}
\hline methylsuccinate & 10.62 & 0.00 & 10.39 & 7.90 & 12.32 & \\
\hline $\mathrm{N}$-acetylisoleucine & 1.84 & -2.68 & 1.54 & 1.12 & 1.88 & \\
\hline valine & 2.34 & 0.37 & 0.33 & 1.83 & 1.25 & \\
\hline 5-aminovalerate & 11.04 & 9.51 & 3.65 & 5.56 & 9.65 & \multirow{7}{*}{ Lysine } \\
\hline fructosyllysine & 0.00 & 12.40 & 14.21 & 12.55 & 14.50 & \\
\hline lysine & 1.14 & -0.74 & -0.31 & 0.49 & -0.25 & \\
\hline N6,N6,N6-trimethyllysine & 1.38 & 0.47 & -0.26 & 0.12 & -0.47 & \\
\hline N6-methyllysine & 3.93 & 1.58 & 2.71 & 0.33 & 4.04 & \\
\hline pipecolate & 1.12 & 0.12 & 0.83 & 0.59 & 1.66 & \\
\hline saccharopine & 1.47 & -8.85 & -1.00 & 0.50 & -0.24 & \\
\hline cysteine & -0.05 & -0.03 & -1.34 & -12.87 & -12.87 & \multirow{7}{*}{$\begin{array}{l}\text { Methionine, } \\
\text { Cysteine, and } \\
\text { Taurine }\end{array}$} \\
\hline cystine & 0.19 & -2.13 & -1.07 & 1.15 & -0.86 & \\
\hline hypotaurine & -3.27 & -1.65 & -12.83 & -1.97 & -0.90 & \\
\hline methionine & 1.95 & 0.39 & -0.07 & 1.33 & 1.07 & \\
\hline methionine sulfoxide & 1.78 & 0.85 & 0.18 & 1.18 & 1.11 & \\
\hline S-adenosylhomocysteine (SAH) & -3.23 & -0.51 & -2.00 & -3.31 & -2.59 & \\
\hline taurine & 0.13 & -0.55 & -2.35 & -1.05 & -0.48 & \\
\hline 1-methylnicotinamide & 11.89 & 0.00 & 11.65 & 8.43 & 0.00 & \multirow{9}{*}{$\begin{array}{l}\text { Nicotinate and } \\
\text { Nicotinamide }\end{array}$} \\
\hline $\begin{array}{l}\text { N1-Methyl-2-pyridone-5- } \\
\text { carboxamide }\end{array}$ & 1.31 & -0.48 & 0.09 & -0.03 & 1.04 & \\
\hline $\begin{array}{l}\text { N1-Methyl-4-pyridone-3- } \\
\text { carboxamide }\end{array}$ & -1.77 & -1.36 & -1.21 & -10.76 & -1.29 & \\
\hline $\mathrm{NAD+}$ & -10.43 & 1.14 & -11.15 & 0.53 & 0.43 & \\
\hline $\mathrm{NADH}$ & -10.09 & 0.27 & -1.97 & -0.28 & 0.46 & \\
\hline nicotinamide & 3.40 & -11.04 & 2.14 & 0.40 & -11.04 & \\
\hline nicotinamide ribonucleotide (NMN) & -0.95 & 1.76 & 0.51 & -1.62 & 0.85 & \\
\hline N'-methylnicotinate & 1.53 & 0.36 & 0.69 & 0.31 & 1.46 & \\
\hline quinolinate & 15.15 & 0.00 & 14.74 & 0.00 & 0.00 & \\
\hline 6-phosphogluconate & -0.42 & 0.47 & 0.03 & -9.11 & -0.94 & \multirow{3}{*}{$\begin{array}{c}\text { Pentose } \\
\text { Phosphate (PPP) }\end{array}$} \\
\hline gluconate & -3.83 & -4.22 & -3.03 & -2.91 & -2.62 & \\
\hline glycerate & 1.20 & 0.38 & 1.20 & -3.36 & 1.93 & \\
\hline CDP-ethanolamine & -3.07 & 0.55 & -9.49 & -0.63 & -0.82 & \multirow{4}{*}{ Phospholipid } \\
\hline choline & 2.17 & -0.10 & 0.54 & -6.87 & -0.42 & \\
\hline glycerophosphorylcholine (GPC) & -2.17 & -1.53 & -0.49 & -0.35 & -0.54 & \\
\hline glycerophosphoethanolamine (GPE) & -0.68 & -1.98 & -1.67 & -5.46 & -4.01 & \\
\hline
\end{tabular}


Polarized Phenotype Compared to the Parent, Resting МФ Phenotype (M0). Pathway

categories and metabolites correspond to the data pictures in Figure 9. Heatmap scale (-8 to 8$)$ is colored from blue to green.

\begin{tabular}{|c|c|c|c|c|c|c|}
\hline glycerophosphoinositol GPI) & 12.18 & 7.46 & 10.98 & 0.00 & -1.71 & \\
\hline glycerophosphoserine (GPS) & 11.86 & 0.00 & 12.30 & 7.33 & 3.03 & \\
\hline phosphocholine & -0.12 & 0.61 & -1.62 & -0.34 & -0.13 & \\
\hline phosphoethanolamine (PE) & 0.09 & -0.07 & -1.14 & 0.05 & 0.02 & \\
\hline trimethylamine $\mathrm{N}$-oxide (TMAO) & 1.93 & 0.93 & 1.01 & 0.82 & 1.79 & \\
\hline 5-methylthioadenosine (MTA) & -0.61 & 1.76 & -3.46 & 0.16 & 1.45 & \multirow{4}{*}{ Polyamine } \\
\hline $\mathrm{N}($ '1)-acetylspermidine & 13.85 & 0.00 & 12.93 & 8.62 & 0.00 & \\
\hline putrescine & 0.76 & -11.15 & -1.61 & -0.19 & -11.15 & \\
\hline spermidine & -10.56 & 0.55 & -2.06 & 0.71 & 1.26 & \\
\hline adenine & -0.70 & 0.53 & -11.56 & -0.38 & -0.96 & \multirow{17}{*}{ Purine } \\
\hline adenosine & 4.02 & 4.45 & 5.03 & 5.18 & 5.66 & \\
\hline adenosine diphosphate (ADP) & 0.36 & 1.22 & -10.01 & -1.64 & -1.07 & \\
\hline adenosine monophosphate (AMP) & 4.66 & 4.35 & 0.54 & 3.66 & 3.85 & \\
\hline AICA ribonucleotide (AICAR) & 0.22 & -2.31 & -0.70 & 0.50 & -1.76 & \\
\hline allantoin & 2.23 & 1.88 & -1.68 & 3.05 & 3.45 & \\
\hline guanine & -0.68 & -2.76 & -5.24 & -13.44 & -13.44 & \\
\hline guanosine & -3.16 & -2.93 & -2.26 & -2.75 & -3.04 & \\
\hline guanosine 5'- diphosphate (GDP) & 2.21 & -8.31 & 0.89 & -8.31 & -8.31 & \\
\hline guanosine monophosphate (GMP) & -0.69 & -0.50 & -3.57 & -2.30 & -2.30 & \\
\hline hypoxanthine & -0.13 & -0.92 & -2.98 & -12.95 & -5.89 & \\
\hline inosine & -5.42 & -2.62 & -14.05 & -13.32 & -13.32 & \\
\hline inosine 5'-monophosphate (IMP) & -1.77 & -0.60 & -13.00 & -12.27 & -12.27 & \\
\hline urate & 2.91 & 1.67 & 2.40 & 1.55 & 3.53 & \\
\hline xanthine & 0.04 & -11.08 & 1.28 & 0.60 & -1.35 & \\
\hline xanthosine & -1.04 & 1.01 & -10.52 & -2.77 & -7.21 & \\
\hline xanthosine 5'-monophosphate (XMP) & -2.13 & -0.77 & -7.99 & -11.17 & -7.21 & \\
\hline 3-aminoisobutyrate & 2.35 & -0.61 & 0.64 & -1.61 & 0.14 & \multirow{9}{*}{ Pyrimidine } \\
\hline 3-ureidopropionate & 10.72 & 11.13 & 0.00 & 9.20 & 10.79 & \\
\hline 5,6-dihydrouridine & 3.72 & 3.10 & 2.87 & 2.61 & 3.98 & \\
\hline beta-alanine & -1.66 & -1.54 & -6.16 & -0.71 & -1.03 & \\
\hline cytidine diphosphate (CDP) & -7.89 & 3.05 & -2.12 & 1.81 & 1.83 & \\
\hline cytidine-5'-monophosphate (CMP) & -1.15 & 1.01 & -2.30 & -0.44 & -0.16 & \\
\hline cytidine triphosphate (CTP) & -10.05 & 0.13 & -10.78 & -0.40 & -1.47 & \\
\hline cytidine & 0.87 & 0.20 & -1.10 & 0.42 & 0.69 & \\
\hline cytosine & 11.77 & 0.00 & 9.48 & 0.00 & 0.00 & \\
\hline
\end{tabular}


Polarized Phenotype Compared to the Parent, Resting МФ Phenotype (M0). Pathway

categories and metabolites correspond to the data pictures in Figure 9. Heatmap scale (-8 to 8$)$ is colored from blue to green.

\begin{tabular}{|c|c|c|c|c|c|c|}
\hline orotate & -2.34 & 1.08 & -10.53 & 0.94 & 1.61 & \\
\hline orotidine & -2.45 & -0.05 & -10.06 & -0.74 & 0.55 & \\
\hline pyridoxine & 1.67 & -1.99 & 0.33 & 1.84 & 1.66 & \\
\hline uridine 5'-diphosphate (UDP) & -4.89 & 1.47 & -10.94 & 0.59 & 0.30 & \\
\hline uridine 5'-monophosphate (UMP) & -10.73 & 0.62 & -6.68 & -1.25 & -0.33 & \\
\hline uracil & -0.24 & -2.99 & -12.31 & -11.59 & 3.44 & \\
\hline uridine & -1.13 & -1.73 & 0.56 & -0.68 & -0.67 & \\
\hline uridine 5'-triphosphate (UTP) & -11.38 & -0.50 & -12.10 & -0.59 & 8.69 & \\
\hline sphinganine & -0.67 & -3.03 & -1.00 & -0.98 & -1.41 & \multirow{2}{*}{ Sphingomyelin } \\
\hline sphingosine & -0.85 & -4.72 & -0.47 & -0.19 & -1.89 & \\
\hline alpha-ketoglutarate & -0.95 & -1.58 & -0.48 & -0.76 & -1.52 & \multirow{7}{*}{ TCA } \\
\hline citrate & -1.24 & -0.51 & -3.71 & 0.20 & 0.50 & \\
\hline fumarate & -0.50 & 0.05 & -4.90 & -1.75 & -1.37 & \\
\hline isocitrate & -12.68 & -2.57 & -2.42 & -2.44 & -2.53 & \\
\hline itaconate & 16.01 & 0.00 & 13.70 & 0.00 & 0.00 & \\
\hline malate & -0.68 & 0.33 & -14.14 & -2.53 & -1.71 & \\
\hline succinate & 1.07 & -0.30 & -1.49 & -6.10 & -4.08 & \\
\hline c-glycosyltryptophan & 0.64 & 0.40 & -2.81 & 0.22 & 0.58 & \multirow{9}{*}{ Tryptophan } \\
\hline N-formylanthranilic acid & 3.22 & -9.40 & 2.51 & -3.90 & -9.40 & \\
\hline indoleacetate & 1.85 & 0.75 & 0.89 & 0.74 & 2.05 & \\
\hline indolelactate & -0.14 & 2.14 & -1.39 & -1.26 & 1.24 & \\
\hline indolepropionate & 4.99 & 4.34 & 4.40 & 3.64 & 6.04 & \\
\hline kynurenate & 10.38 & 0.00 & 10.77 & 0.00 & 6.92 & \\
\hline kynurenine & 14.49 & 0.00 & 14.35 & 0.00 & 0.00 & \\
\hline serotonin & 2.11 & 0.22 & -0.22 & -0.36 & 1.20 & \\
\hline tryptophan & -13.91 & -0.52 & -14.63 & 0.14 & 0.10 & \\
\hline 1-pyrroline-5-carboxylate & -2.46 & -1.65 & -10.69 & -0.03 & -2.34 & \multirow{9}{*}{ Urea Cycle } \\
\hline 2-oxoarginine & 12.77 & 0.00 & 12.56 & 10.22 & 12.96 & \\
\hline arginine & 0.79 & 0.28 & -0.29 & 0.38 & 0.43 & \\
\hline argininosuccinate & -2.37 & -2.07 & -11.75 & -11.03 & -5.32 & \\
\hline citrulline & 3.38 & 2.16 & 2.09 & 2.47 & 3.00 & \\
\hline hydroxyproline & 1.52 & 0.56 & -0.32 & 0.58 & 0.56 & \\
\hline ornithine & 2.07 & 0.83 & 1.45 & 2.24 & 2.33 & \\
\hline proline & 1.19 & 0.11 & 0.10 & 0.55 & 0.75 & \\
\hline urea & 3.57 & 1.73 & 2.73 & 2.50 & -0.67 & \\
\hline
\end{tabular}

